\title{
La nomination du vizir Ouseramon d'après le papyrus Turin Cat. 1878 vo
}

\author{
Mathilde Frère
}

The powerful vizier Useramun was in office from year 5 to year 28 or 34 of the reign of Tuthmosis III, succeeding his father, Ahmose Aametu. A text referring to his appointment by the king is preserved on the verso of papyrus Turin Cat. 1878. Although this text was already known to Champollion, no indepth study of it has been carried out since the publication of its first copy by Pleyte and Rossi in 1869-1876 and of its first transcription and translation by Helck in 1955. Here the author presents a new facsimile and study of the text, clarifying several palaeographic issues and shedding further light on the meaning of this rather enigmatic composition, which has no known parallel in Egyptian literature, but echoes a scene in Useramun's tomb, TT 131, showing the vizier Ahmose Aametu introducing his son Useramun to the king, who appoints him as his father's successor. The text on the Turin papyrus also raises questions about the theme of circumcision and the use of the metaphor of silver and gold to designate the vizier and the king, respectively. The composition is a Ramesside historical fiction, most likely written during the first half of the Twentieth Dynasty, when the memory of the vizier Useramun evidently still loomed large.

\section{ملخص البحث:}

تولى الوزير القوي "أوسر -أمون منصبه من العام الخامس وحتى العام الثامن والعشرون أو الرابع والثلاثون من عهد تحتمس الثالث، الذي خلف والده أحمس-عامتو. كما يشير النص على ظهر البردية المحفوظة بالمتحف المصري بتورينو رقم ( Turin (Cat 1878 على تتصيبه من قِبل الملك. على الرغم من أن هذا النص كان معروفاً لدى شامبليون، إلا أنه لم يتم إجراء أي دراسة متعدقة له منذ نشر نسخته الأولى من قبل بليت و روسي من عام 11 1 إلى عام 1 1 و و منذ أول ترجمة ونقل لفظ النص التي أجراها هيلك في عام 900 ـ يقدم المؤلف هنا نَسخاً ودراسة جديدة للنص، كما أنه يوضح العديد من المسائل المتعلقة بالكتابة اليدوية القديمة (الباليوجرافي) ويلقي مزيداً من الضوء على معنى هذه المقالة الغامضة إلى حد ما، والتي لا مثيل لها في الأدب المصري. يُحاكي النص مشهداً من مقبرة أوسر -أمون، المقبرة الطيبية رقم 131 TT، يظهر فيه الوزير أحمس -عامتو وهو يُقدم ابنه أوسر -أمون للملك، الذي يعينه خليفةً لوالده. يثير أيضاً النص الوارد بالبردية المحفوظة بالمتحف المصري بتورينو أسئلة حول موضوع الختان وإستخدام إستعارة الفضة والذهب عند تتصيب الوزير والملك على التوالي. المقالة عبارة عن رواية تاريخية تعود لعصر الرعامسة، كُتبتِ على الأرجح خلال الفترة الأولى لحكم الأسرة العشرين، عندما كانت ذكرى الوزير أوسر -أمون لا تزال تلوح في الأفق.

Célèbre vizir de la XVIII dynastie, Ouseramon exerça cette fonction à la suite de son père, Âhmosis Âamtjou, durant le règne conjoint d'Hatchepsout et de Thoutmosis III, puis sous celui, autonome, du jeune roi. Ce haut dignitaire est bien connu grâce à ses deux tombes situées dans la nécropole thébaine de Cheikh Abd el-Gournah (TT 61 et TT 131), et par de nombreux autres monuments et documents ${ }^{1}$. Il est, en outre, le seul vizir de la XVIII ${ }^{\mathrm{e}}$ dynastie pour lequel la date exacte de sa nomination à ce poste le $1^{\mathrm{er}}$ jour $\mathrm{du} 1^{\mathrm{er}}$ mois de la saison-akhet en l'an 5 de Thoutmosis III - est connue puisque mentionnée 
au verso du papyrus Turin Cat. 1878. Bien qu'il soit d'une grande importance historique et ait déjà été connu de Champollion ${ }^{2}$, ce texte n'a paradoxalement été que très peu étudié dans son ensemble ${ }^{3}$.

Depuis la première copie et traduction partielle par Pleyte et Rossi en 1869-1876 (le dessin occupe la première planche de leur ouvrage) $(\mathrm{Pl} .1)^{4}$, les mentions du texte sont assez marginales. Une entrée existe dans le catalogue du musée de Turin de Fabretti, Rossi et Lanzone de $1882^{5}$ ainsi que dans celui de Bellion de $1987^{6}$. Il est répertorié dans les fiches manuscrites du Wörterbuch, au moins pour les entrées $m \underline{t} z$ et $d h n(\text { ? })^{7}$. Quant aux transcriptions, les deux seules publiées sont dues à Helck ${ }^{8}$. Elles ont cependant été effectuées à partir de la copie de Pleyte et Rossi. Černý en a également réalisé une dans ses Notebooks ${ }^{9}$.

Helck $^{10}$, en 1955, 1958 et 1961, et Dziobek, en $1998^{11}$, ont proposé une traduction complète du texte, accompagnée de quelques commentaires. Enfin, Dorman fournit un bref commentaire historique du texte en 1988 dans son ouvrage consacré à Senenmout, ainsi que, quelques années plus tard, au sein de l'ouvrage collectif dédié au règne de Thoutmosis III paru en $2006^{12}$. À l'exception des travaux précédemment cités, les autres traces de ce texte dans la littérature égyptologique se limitent à quelques citations ${ }^{13}$.

Le service de la collection des papyrus de la Fondazione Museo delle Antichità Egizie di Torino a, pour sa part, réalisé en novembre 2017 les seules images numérisées du papyrus existantes à ce jour (Fig. 2$)^{14}$. Ces dernières ont permis la réalisation d'un fac-similé fiable, au moyen du logiciel Adobe Photoshop (Fig. 3$)^{15}$, ainsi que la résolution de plusieurs problèmes paléographiques. La signification globale du texte s'en trouve améliorée bien que plusieurs passages demeurent assez obscurs. De façon plus générale, ce texte est singulier et demeure sans véritable parallèle dans la documentation égyptienne connue.

\section{Présentation, description et étude préliminaire de l'ensemble CGT $54018 \mathrm{r}^{\circ}$ et $\mathbf{v}^{\circ}$}

Le papyrus Turin Cat. 1878 est conservé dans la collection du Museo Egizio de Turin depuis 1824. Il appartient à un ensemble de fragments regrou- pés sous le numéro CGT 54018 (= Turin Cat. 1878 + Cat. $2027^{16}$ + Cat. $1968 / 002$ + Cat. 1890 + quatre fragments a-d sans numéro; Pls. 2-5). Cet ensemble est constitué de deux grands fragments principaux et de trois petits morceaux (frag. b-d). Le grand fragment principal $\mathrm{n}^{\circ} 1$ (33,6 cm de long sur $20 \mathrm{~cm}$ de haut) rassemble la partie sans numéro a, le fragment Cat. 1878 ainsi que deux morceaux plus petits et sans numéro (frag. b-c), ne se raccordant pas directement avec le grand fragment $n^{\circ} 1^{17}$. Les numéros Cat. 2027 + Cat. 1968/002 + Cat. 1890 forment le grand fragment principal $\mathrm{n}^{\circ} 2(42,4 \mathrm{~cm}$ de longueur sur $20 \mathrm{~cm}$ de hauteur) auxquels il faut ajouter le troisième petit morceau (frag. d) qui leur est discontinu (6,1 cm de hauteur sur 1,8 cm de largeur). L'ensemble de ces pièces sont très probablement issues de la collection de Drovetti ${ }^{18}$. Bien que nous ne connaissions pas le lieu exact de leur découverte, Deir al-Medineh est l'hypothèse la plus vraisemblable.

\subsection{Description}

Les fragments de papyrus appartenant au CGT $54018 \mathrm{r}^{\circ}$ (= Turin Cat. $1878 \mathrm{r}^{0}+$ Cat. $2027 \mathrm{r}^{\circ}+$ Cat. 1968/002 $\mathrm{r}^{\circ}+$ Cat. $1890 \mathrm{r}^{\circ}+$ les quatre fragments a-d sans numéro) mentionnent l'un des exemples connus du Grand Hymne à Hâpi (strophes II,5-XIV,10 et colophon ; Pls. 2, Pls. 4) et sont datés de la première moitié de la XX dynastie ${ }^{19}$. La notice du Lexikon der Ägyptologie consacrée aux papyrus de Turin est la première à avoir mis en relation ces fragments avec le document relatant la nomination présente au verso ${ }^{20}$, et les images numérisées recto et verso du papyrus confirment ce lien : le recto du pap. Turin Cat. 1878 I,1-10-II,1-10 comporte les strophes II,5-IX,8 du Grand Hymne à Hâpi, et le texte de la nomination d'Ouseramon est écrit au verso.

Le verso de l'ensemble CGT 54018 (= Turin Cat. 1878 $\mathrm{v}^{0}+$ Cat. $2027 \mathrm{v}^{0}+$ Cat. 1968/002 vo + Cat. $1890 \mathrm{v}^{0}$ + les quatre fragments a-d sans numéro ; Pls. 3, 5), quant à lui, contient au moins cinq textes inscrits à l'encre noire, et cela par cinq scribes différents.

Le texte $\mathbf{n}^{0} 1$ (= frag. a.1 - inédit ; $13 \mathrm{~cm}$ de hauteur sur une longueur conservée de $6 \mathrm{~cm}$ ) est écrit sur la partie droite du fragment principal $n^{\circ} 1$, d'une écriture ferme et maîtrisée. Notons qu'il est disposé dans le sens inverse par rapport aux textes nos $2-5$. 
Après un premier examen visuel à partir des images numérisées, ce texte fragmentaire pourrait être funéraire, rituel, hymnique ou médico-magique. Mentionnons enfin que la partie conservée du texte n'est ni rubriquée ni versifiée.

Le texte $\mathbf{n}^{\circ} \mathbf{2}$ ( = frag. a.2 - inédit ; 4,4 cm de hauteur sur 8,4 $\mathrm{m}$ de longueur) a été rédigé en haut de la partie centrale du fragment, d'une écriture rapide et de petites dimensions. La paléographie permet une datation ramesside. Il s'agit d'un texte comptable de trois lignes mentionnant " l'an $1,2^{\mathrm{e}}$ mois de la saison-chémou, 16 jour » d'un souverain anonyme, ainsi que la quantité considérable de 290 (ou 390 ?) dében de cuivre.

Le texte $n^{0} 3$, relatif au vizir Ouseramon (= pap. Turin Cat. $1878 ; 20 \mathrm{~cm}$ de long sur $15 \mathrm{~cm}$ de haut), se répartit du centre vers la partie gauche du fragment principal no 1 . Il a été tracé par un scribe d'une écriture ample et rapide. La paléographie est ramesside (début XX $X^{e}$ dynastie, voir infra § 4.2) et le texte n’est ni rubriqué ni versifié.

Neuf lignes fragmentaires sont conservées. L'interruption du texte à gauche étant très nette, d'autres morceaux de ce document pourraient ainsi être présents dans les réserves du musée de Turin, dont le raccord aujourd'hui manquant ; hypothèse renforcée par le fait que la suite du rouleau de papyrus est conservée (frag. b-c + Cat. $2027 \mathrm{v}^{0}+$ Cat. 1968/002 v + Cat. $1890 \mathrm{v}^{0}$ + frag. d ; Pls. 3, 5). Il est également à noter que, depuis le relevé réalisé par Pleyte et Rossi au cours de la seconde moitié du XIX ${ }^{e}$ siècle, l'extrémité gauche du papyrus s'est dégradée, faisant ainsi disparaître les signes de la fin du cartouche de Thoutmosis III (Pls. 1, 3 et Fig. 2).

Bien que le document soit fragmentaire, et en supposant que le texte complet ne se poursuivait pas au-delà de ces neuf lignes, d'après nos calculs sur la base de la taille des signes ainsi que sur l'emplacement du texte $\mathrm{n}^{\circ} 4$, nous pouvons estimer qu'environ $70 \%$ du texte original est conservé et que les restitutions suggérées couvrent à peu près $15-20 \%$. Par conséquent, près de $85-90 \%$ du texte original est actuellement exploitable et environ 10-15\% serait encore totalement inconnu (Pl. 5).
Les apparentes traces d'un texte palimpseste écrit à l'encre noir et rouge, visibles sur les images numérisées, appartiennent en réalité au Grand Hymne à Hâpi inscrit sur le recto, le feuillet de papyrus étant particulièrement fin et transparent ; les seules traces réelles de palimpseste se situent à gauche au-dessus du texte ${ }^{21}$.

Le texte $\mathbf{n}^{\circ} \mathbf{4}$ ( = frag. c et b + pap. Turin Cat. $2027 \mathrm{~V}^{\circ}$ I - inédit) est consigné sur les deux petits fragments mentionnés plus haut (frag. $c$ et $b$ : 3,6 et $7 \mathrm{~cm}$ de hauteur) et se poursuit sur le pap. Turin Cat. 2027 $\mathrm{V}^{\circ}$ I qui est le début du grand fragment $\mathrm{n}^{\circ} 2(8,4 \mathrm{~cm}$ de longueur sur 15,6 cm de hauteur). Il a été tracé par un scribe d'une écriture ample et rapide. Un seul point de versification est actuellement perceptible à la ligne sept. La nature exacte de ce texte littéraire n’a pas encore été établie.

Enfin, le texte $\mathbf{n}^{0} \mathbf{5}$ (= pap. Turin Cat. $2027 \mathrm{~V}^{0}$ II + Cat. 1968/002 vo + Cat. 1890 vo I-II + frag. d - inédit) est inscrit sur la suite du grand fragment $n^{\circ} 2$ (33 cm de longueur sur 16,7 cm de hauteur) ainsi que sur le troisième petit morceau (frag. d). Ils comporteraient un passage d'un texte littéraire versifié, peut-être l'Enseignement fait par le scribe Chéty fils de Douaouf pour son fils Pépy (aussi appelé Satire des Métiers) selon l'identification de Roccati ${ }^{22}$. Toutefois, après vérification du texte d'après les images numérisées et en comparant avec les différentes versions de l'Enseignement de Chety ${ }^{23}$, le texte ne semble pas appartenir à cette oeuvre.

Il est à noter que le dessin d'une partie du fragment Cat. 1968/002 vo ainsi que quelques commentaires du texte sont présents dans l'ouvrage de Pleyte et Rossi $^{24}$. Il s'agit, pour eux, d'un fragment d'hymne daté de la XXe dynastie. De plus, une fiche manuscrite du Wörterbuch nous fournit une transcription de ce même passage, identifié comme étant un texte littéraire du Nouvel Empire ${ }^{25}$. Ces deux travaux n'apportent pas, cependant, de plus amples renseignements concernant l'identification précise de la nature de cette production littéraire.

À notre connaissance, les textes $n^{\text {os }} 1,2$ et 4 ne sont pas mentionnés dans la littérature égyptologique, et aucun rapport n’a donc été établi avec le fragment de papyrus numéroté Cat. 1878. De plus, la recon- 


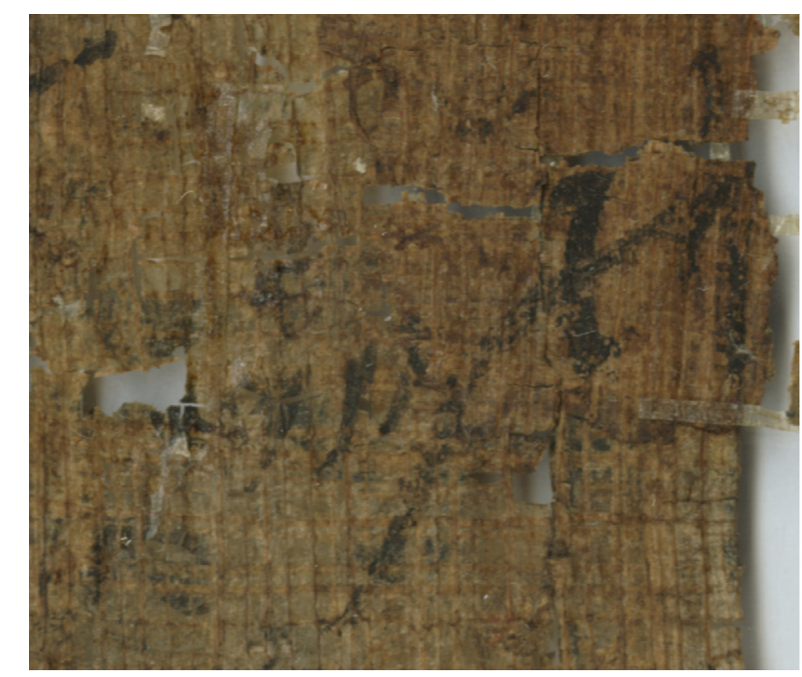

a

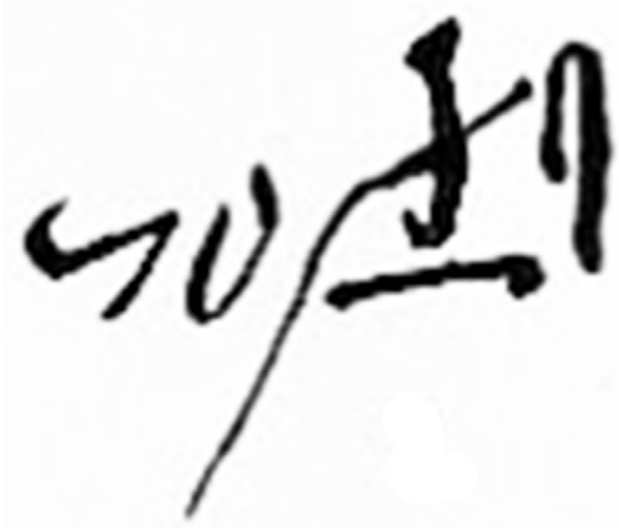

$b$

Fig. 1 : Détail du papyrus Turin CGT 54018 vº, frag. a.2 (texte n²).

a : Image numérisée : Museo Egizio.

b : Dessin tiré de Pleyte et Rossi, Les papyrus hiératiques de Turin, II, 18691876, pl. I.

naissance du texte comptable (texte $\mathrm{n}^{\circ} 2$ = frag. a.2) permet de comprendre l'origine des quelques signes apparaissant hors du texte relatif à Ouseramon sur la planche de Pleyte et Rossi ${ }^{26}$, et qui appartiennent donc à cette inscription (Pl. 1 et Figs. 1a-b, Fig. 2). Cette constatation nous révèle également qu’à leur époque, le papyrus n'était pas recollé tel qu'il l'est actuellement (Pl. 5).

\section{2. Étude préliminaire}

Notre étude préliminaire de l'ensemble des fragments du volumen CGT 54018 apporte quelques premiers éléments de réponse concernant la matérialité de même que l'histoire du manuscrit tout en proposant plusieurs hypothèses et perspectives de recherche $^{27}$ (Pls. 4-5).

Signalons que cet examen n'a pu être effectué uniquement qu'à partir des images numérisées aimablement communiquées par le service de la collection des papyrus de la Fondazione du Museo Egizio de Turin. Certains aspects restent donc de ce fait délicats à appréhender en l'état actuel de conservation globale de l'objet ainsi qu'en raison des nombreuses données demeurant, pour le moment, partielles ou imprécises étant donné que le document n'a pas encore été étudié dans sa totalité de manière approfondie.
Tout d'abord, d'après l'observation visuelle des fragments ainsi que de la définition matérielle des deux faces d'un rouleau de papyrus et de leurs propriétés comme supports de textes ${ }^{28}$, il appert que les appellations « recto » et « verso » attribuées antérieurement correspondent au recto et verso réel du papyrus.

Deuxièmement, les mesures de l'objet de même que notre cartographie de la distribution des textes nous fournissent de précieux renseignements concernant l'assemblage des différents fragments conservés ainsi que sur la restitution de cette portion du rouleau dans son état d'origine.

Étant donné qu'un long morceau continu du feuillet original est conservé sur le grand fragment numéroté Cat. 1878 + frag. a.1-2, nous estimons d'après nos calculs fondés sur la longueur de la section de l'Hymne inscrite sur cette portion -, que la longueur totale de ce feuillet était d'environ $32 \mathrm{~cm}$. En appliquant de manière théorique cette donnée à l'ensemble CGT 54018, chacun des feuillets de ce volumen devait de ce fait mesurer autour d'une trentaine de centimètres de longueur. De plus, la hauteur totale étant conservée à plusieurs endroits, nous sommes en mesure d'affirmer que les feuillets ont une hauteur de $20 \mathrm{~cm}$ (soit 0,38 coudée = 2,67 mains). Cette mesure, correspondant au demi-rou- 
leau (half-roll), était celle couramment utilisée tout au long de l'époque ramesside ${ }^{29}$.

Dans le cas présent où la longueur des feuillets de notre papyrus est estimée à 30-32 cm (soit 0,570,61 coudée $=4-4,27$ mains) et en prenant en considération le fait qu'un volumen comportait généralement vingt feuillets ${ }^{30}$, le support de communication étudié ici devait alors au moins mesurer au total entre 6 et 6,40 $\mathrm{m}$ de long (soit 11,43-12,19 coudées $=80-85,33$ mains) lors de sa sortie de l'atelier de production.

Par ailleurs, d'après la continuité des sections du texte de l'Hymne à Hâpi inscrit sur l'ensemble du recto ainsi que l'estimation de la longueur des feuillets, il nous est alors possible de déterminer les distances entre chacun des fragments conservés. De plus, puisque deux joints de fabrication (ou kollêseis) sont préservés, nous possédons donc nécessairement les portions d'au moins trois feuillets. Le remontage numérique que nous proposons met en évidence que l'ajout d'un feuillet supplémentaire s'impose en dépit du fait que le joint entre les feuillets $x+2$ et $x+3$ n'a pas encore été retrouvé ( $\mathrm{Pl}$. 4). Nous possédons donc actuellement les bribes de quatre feuillets de ce volumen.

D’après notre cartographie de la distribution du Grand Hymne rédigé sur ce papyrus ( $\mathrm{Pl} .4$ ), le titre et les strophes I,1-II,4 (début) devaient, à l'origine, être présents sur un feuillet supplémentaire. Celui-ci est à insérer à la droite du grand fragment $\mathrm{n}^{\circ}$ 1. L'intégralité de cette oeuvre littéraire était donc très vraisemblablement inscrite sur le recto de l'ensemble CGT 54018 et cela sur cinq feuillets complets, ce qui équivaut à $25 \%$ de la totalité du recto de ce rouleau de papyrus. De ce fait, l'Hymne devait s'étendre sur une longueur totale d'environ 1,50 à 1,60 m, dont $86 \mathrm{~cm}$ sont actuellement partiellement conservés. Ceci étant dit, remarquons que nous ne connaissons pas l'état dans lequel était le rouleau orignal - neuf ou déjà usagé -, et donc la longueur effective de l'objet lorsque le scribe y a inscrit la composition littéraire dédiée au fleuve égyptien.

Troisièmement, l'ensemble CGT 54018 présente une certaine hétérogénéité textuelle en contenant au moins six textes, dont cinq ont été assemblés au verso. Sur cette face-ci, un texte comptable (texte $\mathrm{n}^{\circ}$ 2) est entouré d'un écrit à caractère hymnique, funéraire ou médico-magique (texte $\mathrm{n}^{\circ} 1$ ) ainsi que de trois textes littéraires $\left(\mathrm{n}^{\mathrm{os}} 3,4,5\right)$. La version du Grand Hymne à Hâpi occupe, quant à elle, tout le recto de cette portion du rouleau de papyrus. Cet arrangement montre donc une prédominance d'oeuvres littéraires rédigées par plusieurs scribes $^{31}$.

Un soin particulier a été apporté à la disposition de l'Hymne à la Crue puisque les kollêseis ne sont pas inscrites. De plus, chaque feuillet ne comporte qu'une seule colonne de texte écrit horizontalement. En revanche, la production littéraire $n^{\circ} 5 \mathrm{du}$ verso ainsi que la Nomination d'Ouseramon (texte $\mathrm{n}^{\circ} 3 \mathrm{du}$ verso) ne respectent pas cette organisation élaborée puisque les textes recouvrent totalement les jointures. Nous ne savons pas s'il en est de même ou non pour les oeuvres littéraires $n^{\text {os }} 1$ et 4 du verso étant donné que les kollêseis ne sont pas conservées sur les fragments relatifs à ces textes. Enfin en quatrième et dernier point, évoquons rapidement la question de l'amplitude temporelle de l'utilisation du manuscrit, au moins comme support d'écriture.

La portion du volumen de papyrus étudiée ici contient six textes inscrits, à première vue, par six scribes distincts et cela au cours de la période ramesside. Le support de communication dont il est question a donc été réutilisé à de nombreuses reprises comme support d'écriture pluripersonnel et éventuellement aussi comme outil de lecture.

Le volumen pourrait avoir été fabriqué à la toute fin de la XIXe ou au début de la XX $\mathrm{XX}^{\mathrm{e}}$ dynastie puis avoir été utilisé pendant tout ou partie de la première moitié de cette période, soit pendant 60 voire 70 $a^{32}$. Au premier abord, cette estimation ne paraît pas excessive compte tenu du fait que ce matériau est souple, léger et résistant. De plus, il n'est pas exclu que ce rouleau ait perduré comme support de lecture au-delà de cette époque.

Nous achevons ici notre analyse préliminaire des fragments du papyrus CGT 54018 dans leur ensemble, laissant le soin à de futurs travaux de confronter ainsi que de compléter nos hypothèses. La suite de notre étude se concentre à présent exclusivement sur le texte de la Nomination du vizir Ouseramon (pap. Turin Cat. $1878 \mathrm{v}^{\circ}$ ). 


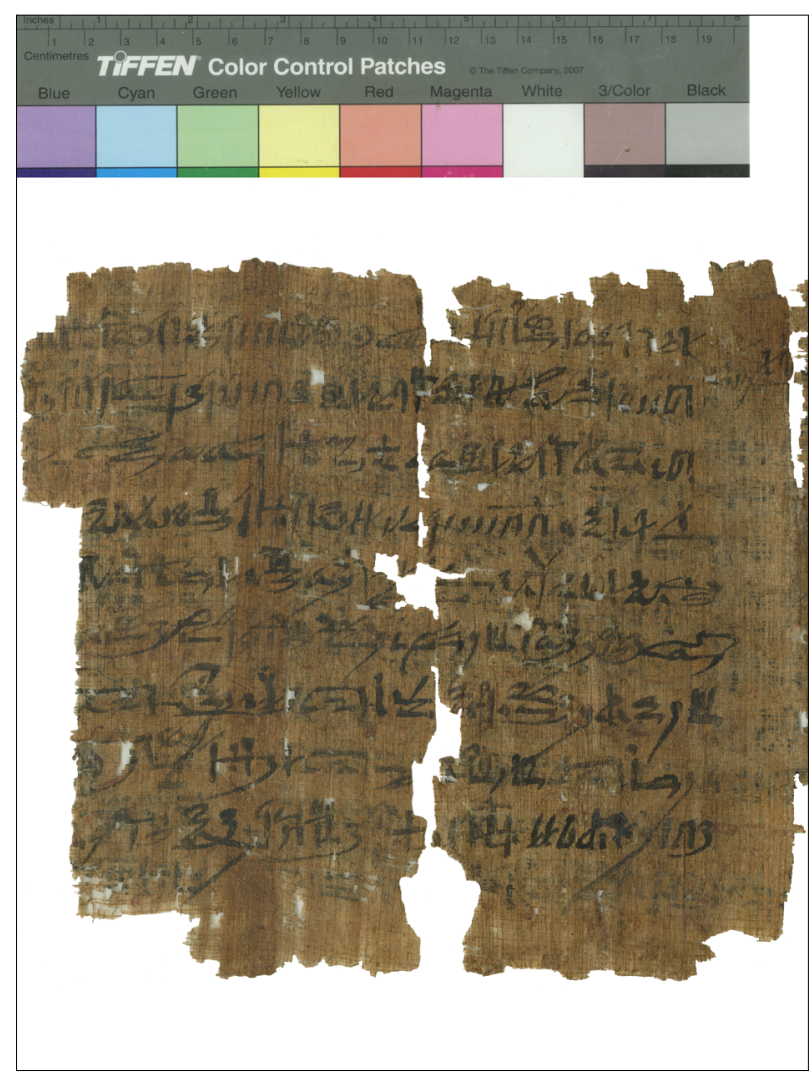

Fig. 2 : Pap. Turin Cat. 1878 vº I. 1-9. Image numérisée : Museo Egizio.

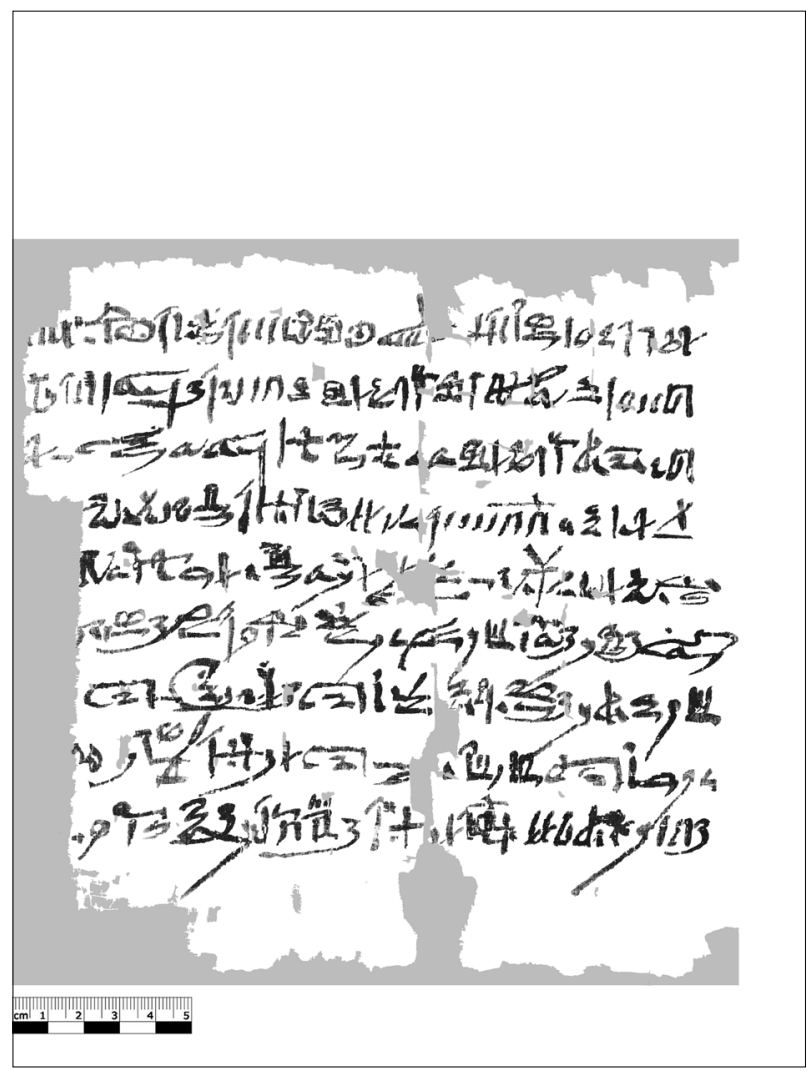

Fig. 3 : Fac-similé du pap. Turin Cat. 1878 vº, I. 1-9. Fac-similé de l'auteur à partir de l'image numérisée communiquée par le Museo Egizio.

\section{Traduction du papyrus Turin Cat. 1878 vo, I. 1-9}

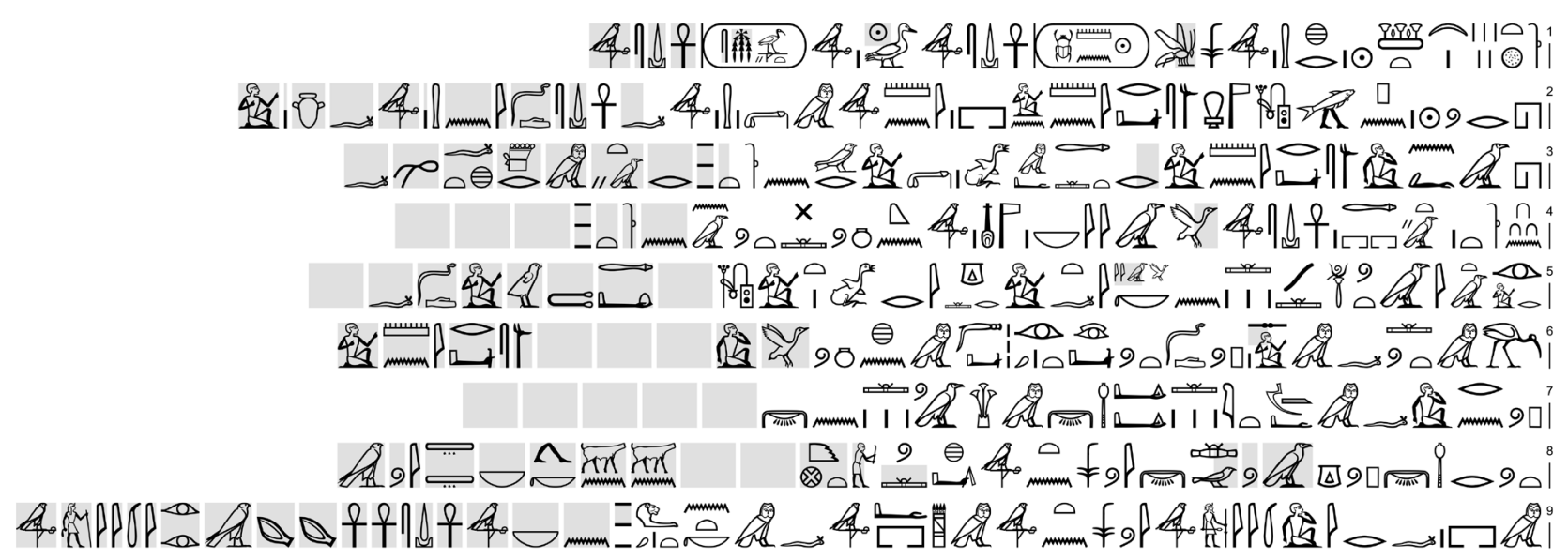

Fig. 4 : Transcription hiéroglyphique du pap. Turin Cat. 1878 vo I. 1-9. Réalisée avec l'éditeur de textes JSesh (version 6.5 .5 [2015]), développé par S. Rosmorduc (http://jsesh.qenherkhopeshef.org/fr).

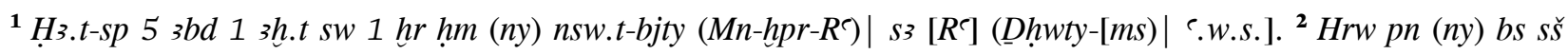

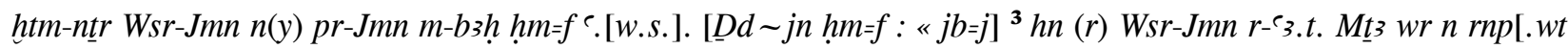

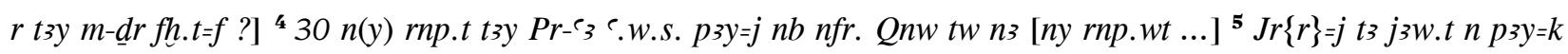

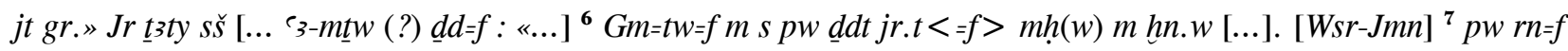

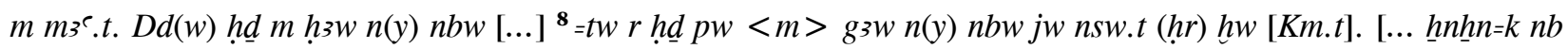

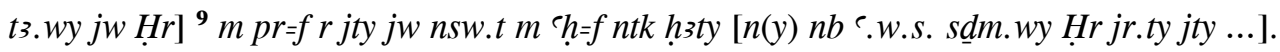


${ }^{1}$ An $5,1^{\text {er }}$ mois de la saison-akhet, $1^{\text {er }}$ jour ${ }^{\mathrm{a}}$, sous la majesté du roi de Haute et Basse-Égypte (Menkheperrê)| le fils de [Rê] (Thout[mosis)| v.s.f.]. ${ }^{2}$ En ce jour de l'initiation ${ }^{\mathbf{b}}$ du scribe du sceau divin ${ }^{\mathbf{c}}$ Ouseramon du domaine d’Amon au-devant de Sa Majesté v.[s.f.]. [Sa Majesté a dit : «[J']approuve grandement ${ }^{\mathbf{3}}$ Ouseramond. Le phallus, [depuis] combien de temps [a-t-il été défait ? ?] $^{\mathbf{4}}$ Cela fait 30 ans, ô Pharaon v.s.f. mon maître parfait ${ }^{f}$ Nombreuses sont [les années ...]. ${ }^{\mathbf{5}}$ Déjà sous ton père, j’ai exercé la fonction ». Quant au vizir, scribe

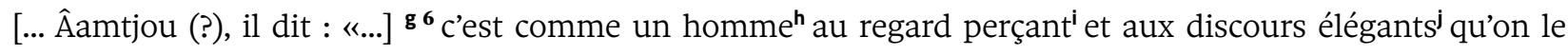
considère [...]. [Ouseramonk ${ }^{\mathrm{z}}{ }^{\mathrm{z}}$ est son nom en vérité. (C'est quelqu'un) qui accorde constamment de l'argent en plus de l'or (/en excès par rapport à l'or) [...]. [C'est] ${ }^{\mathbf{8}}$ vers l'argent qu'il faut [se tourner (/aller)] en l'absence de l'or (/quand l'or manque) lorsque le roi protège [1’Égypte ...]'. [Alors que l'Horus] ${ }^{\mathbf{9}}$ est dans sa demeure, (y) étant souverain, et que le roi est dans son palais [tu t’approches du Maitre du Double-Pays ${ }^{\mathrm{m}}$ ] (puisque) tu es le coeur [du Maître v.s.f., les (deux) oreilles d'Horus, les (deux) yeux du souverainn ${ }^{\text {...] }] » .}$

\section{Commentaires de traduction}

a: Gauthier enregistre une autre date, toutefois avec une certaine réserve : « An 5 (?), $1^{\text {er }}$ mois (?) de la saison-chemou (?), $1^{\text {er }}$ jour » (ḩ.t-sp 5 tpy šmw sw 1), et n'est pas certain de la mention « an $5 »^{33}$.

b: Helck transcrit par deux fois, $s \underline{t} z$ « introduire $»^{34}$, peut-être par effet de contamination avec la Nomination du vizir ${ }^{35}$. Cependant, Pleyte et Černý transcrivent ce passage $b s$ « initier, introduire ${ }^{36}$ (voir infra, § 3.3.1).

‘: Ouseramon n'est désigné par ce titre de « scribe du sceau divin » qu’à trois autres reprises : deux fois dans sa tombe TT 131 ainsi que sur le cône funéraire Davies-Macadam $370^{37}$.

d: Litt. «[Mon coeur] est très penché (vers) Ouseramon », en suivant la restitution proposée par Helck ${ }^{38}$. Dziobek ${ }^{39}$ translitère $h_{3} n_{3}$ « daß doch » et renvoie, à notre avis à tort, au Wb II, 481, 7. Černý translitère $[d] h n$ " promouvoir, désigner, nommer, mettre en place » et renvoie au $\mathrm{Wb} \mathrm{V}, 479,10-11$.

e: Helck restitue un $p w$ dans la lacune. Or, d'après le type de formulation, il serait davantage plausible de restituer $r$ t3y, comme le fait Černý ${ }^{40}$.

La compréhension générale de la ligne 3 est nettement débattue. Le problème repose avant tout sur la différence de lecture d'un seul signe, qui plus est, partiellement en lacune due au fait que le papyrus est détérioré à cet endroit, ainsi que sur la graphie litigieuse d'un mot.

En se fondant uniquement sur le dessin de Rossi, Quack exprime d'importants doutes quant à la proposition de traduction et donc la compréhension de cette phrase par Helck et, au final, la rejette totale- ment. Étant donné qu'il discerne le signe hiératique de l'oeuf, typique de la marque de la filiation, à la place de la préposition $r$ transcrite par Helck, il considère qu'il faut lire : «[...] d'accord (avec)/approuver Ouseramon, fils d'Âhmose (i.e. Âamtjou), combien d'années [...] ? » ${ }^{41}$ (Figs. 5c, 6c). Il estime donc que le nom d'Ouseramon est directement suivi par le nom de son père, prénommé Âamtjou. Il juge que c'est un nom asiatique, et que, dans ce papyrus, il serait écrit sous une forme pseudo-étymologique, comme s'il s'était appelé en égyptien ` $3 t \underline{t} 3$, « avec un grand phallus $»^{42}$. Ainsi, pour Quack, ce document n'atteste pas de la pratique de la circoncision, corrélée de plus à un critère d'âge ainsi qu'à un acte rituel dans le cadre d'une nomination, et doit donc être retiré du corpus documentaire attestant de cet usage.

Au final, il s'agit donc de déterminer à qui appartient, en réalité, ce $m \underline{t} z$ ? Le mot se réfère-t-il au membre viril d'Ouseramon ou bien alors à celui de son père Âamtjou?

Grâce à l'image numérisée et au fac-similé réalisé à partir de celle-ci, nous observons, d'une part, que le ductus correspond davantage au signe hiératique Möller II, 91 ( $r$ ) qu'à celui de Möller II, 238 (l'oeuf) (Figs. 5a-b, 6a-b) ${ }^{43}$. D'autre part, d'après le tableau paléographique présenté en annexe de cet article, ce qu'il reste du signe litigieux est semblable aux extrémités gauches des occurrences des signes $r$ tracés aux lignes 1, 5 (2), 8 et 9 (voir Annexe 1). Au demeurant, remarquons que Černý a également transcrit le signe hiératique problématique par $r$ dans le relevé du texte qu'il a effectué d'après le papyrus original lors de l'une de ses visites au musée de Turin ${ }^{44}$. 

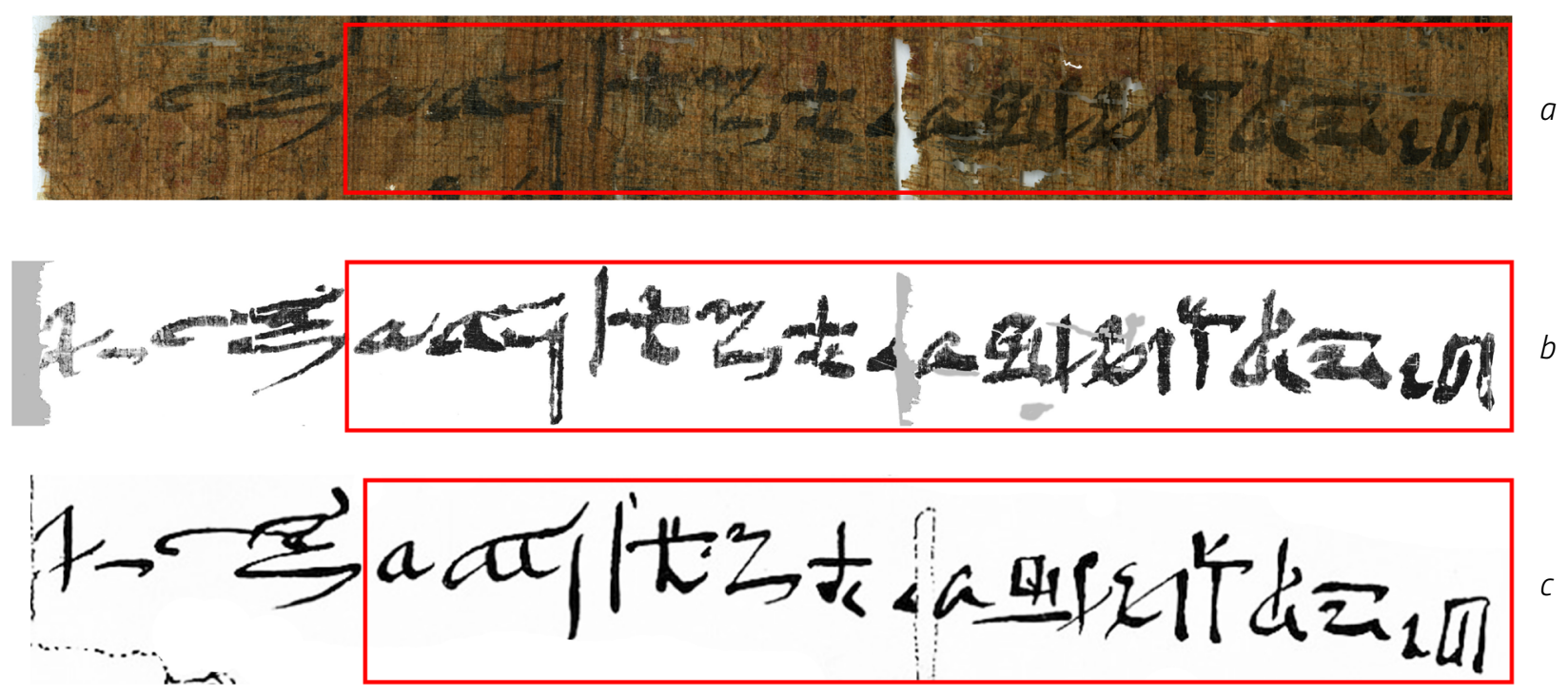

Fig. 5 : Image numérisée, fac-similé et dessin du pap. Turin Cat. 1878 vo I. 3.

$a$ : Image numérisée : Museo Egizio.

$b$ : Fac-similé de l'auteur à partir de l'image numérisée communiquée par le Museo Egizio.

c: Dessin tiré de Pleyte et Rossi, Les papyrus hiératiques de Turin, II, 1869-1876, pl. I.

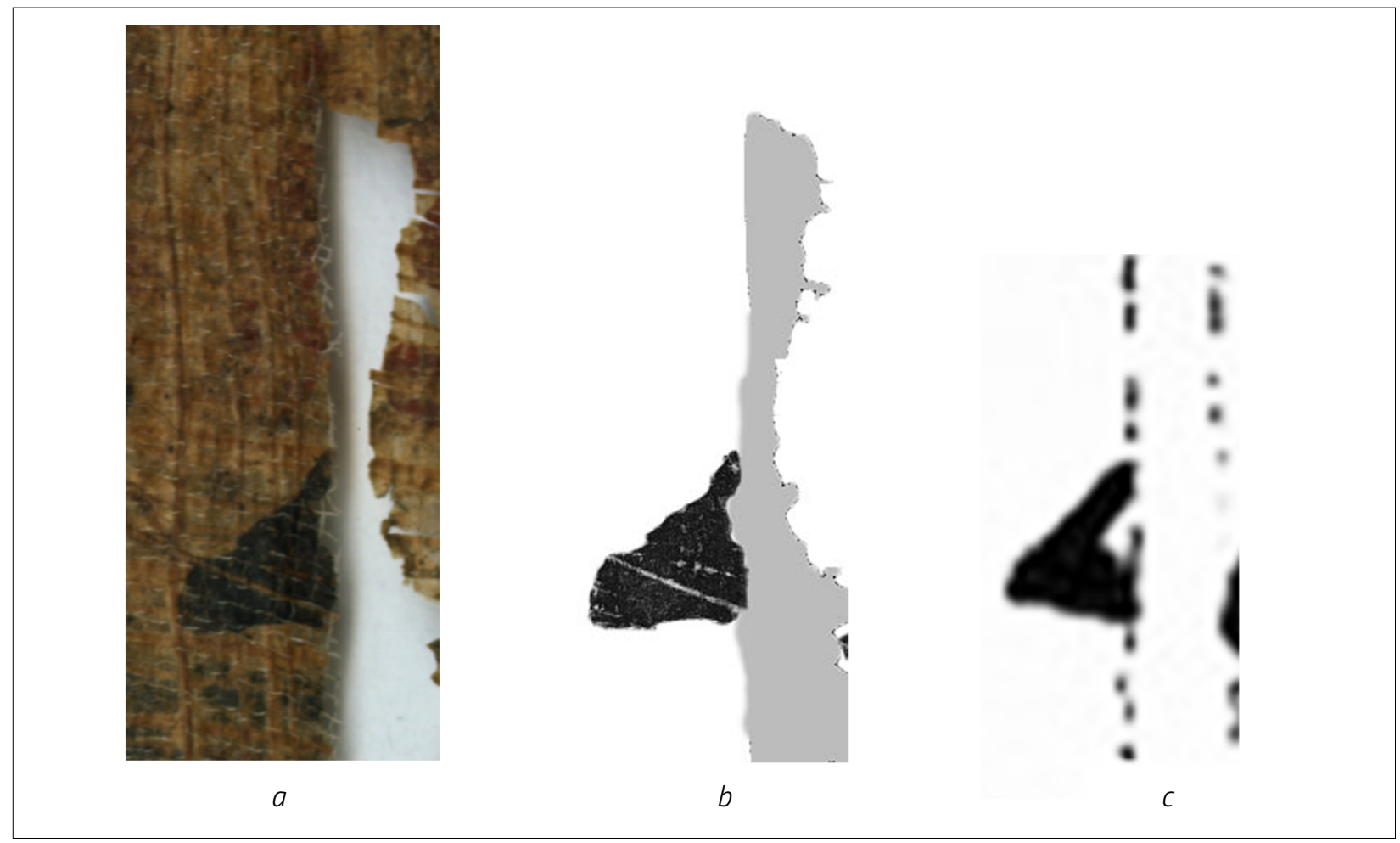

Fig. 6 : Détail du signe problématique du pap. Turin Cat. 1878 vº I. 3 (image numérisée, fac-similé et dessin). $a$ : Image numérisée : Museo Egizio.

b: Fac-similé de l'auteur à partir de l'image numérisée communiquée par le Museo Egizio.

c: Dessin tiré de Pleyte et Rossi, Les papyrus hiératiques de Turin, II, 1869-1876, pl. I.

De plus, ce n’est pas `3 qui est écrit, mais bien `3.t. ${ }^{45}$ Même si l'ajout d'un $t$ " remplisseur d'espace » est une pratique courante au sein de la production textuelle hiératique égyptienne, nous ne pouvons pas pour autant, en revanche, totalement négliger la présence de ce signe.
Puisque le rédacteur semble avoir porté une attention particulière à l'élaboration de ce supposé nom propre sous une forme pseudo-étymologique afin de produire un effet de style recherché, tant graphiquement que du point de vue du sens, pourquoi aurait-il, dans ces conditions, tracé ce signe $t$ totale- 
ment superflu, et ce d'autant plus qu'aucun autre $t$ ayant cette fonction n'est présent dans le texte ?

Faut-il alors considérer que ce $t$ n'est pas un simple " remplisseur d'espace » ? Dans ce cas, l'explication para-étymologique translittérée ne rend pas avec exactitude la proposition égyptienne.

En revanche, l'expression adverbiale r-3.t-(wr.t) "grandement, considérablement, extrêmement, dans une large mesure, à un degré élevé » est, quant à elle, bien attestée dans la documentation égyptienne ${ }^{46}$. C'est d'ailleurs dans ce sens-là que les rédacteurs du Wörterbuch ont compris le passage problématique de notre papyrus puisqu'ils citent ce document dans leurs sources illustrant l'expression $r$ - -3.t. $^{47}$.

Par ailleurs, en admettant l'hypothèse de Quack, nous pouvons nous demander pourquoi une telle graphie pseudo-étymologique, non attestée dans la documentation concernant Âamtjou, aurait été utilisée ici, et cela même plusieurs siècles après le floruit de ce vizir. En effet, pourquoi les scribes du début de la XX dynastie auraient créé de toute pièce cette graphie alors que le nom du père d'Ouseramon, patriarche d'une véritable dynastie vizirale sur trois générations durant la première partie de la XVIII dynastie, était à n'en pas douter encore très présent dans la mémoire collective de l'élite thébaine ramesside ?

De plus, notre texte s'inspire en partie directement de celui de la Nomination gravé dans la tombe d'Ouseramon (TT 131) (voir infra § 4.3). Or, dans l'espace architectural de sa sépulture, cette oeuvre est précisément immédiatement suivie par l'Enseignement d'Âamtjou, toutes deux étant gravées sur le même mur. En outre, à la fois dans la représentation iconographique de la Nomination de même que dans celle de l'Enseignement d'Âamtjou, le père d'Ouseramon est représenté grandeur nature en tant que vizir en titre et affublé des attributs vestimentaires propres à sa fonction (voir infra § 3.1). D’une manière plus générale, son nom est inscrit à plusieurs reprises dans la tombe de son fils ${ }^{48}$.

N'oublions pas, de surcroît, que, malgré l'état actuellement très dégradé de sa tombe, Âamtjou possédait l'une des plus imposantes sépultures situées sur les hauteurs de la colline de la nécropole de Cheikh Abd el-Gournah (TT 83). La monumentale façade à pilier de son complexe funéraire était notamment particulièrement distincte dans le paysage architectural de la nécropole thébaine ${ }^{49}$.

Les scribes de l'époque ramesside avaient de ce fait pleinement accès à la graphie originelle du nom du père de notre vizir. Aucun obstacle technique ni mémoriel ne semble donc avoir pu les restreindre. Par conséquent, ils n’avaient, à priori, aucune raison particulière de recourir à une pseudo-étymologie pour le nom Âamtjou si telle avait été leur intention de le mentionner à la troisième ligne de notre texte. Du reste, remarquons que seul le nom d'Âamtjou aurait été orthographié de manière inhabituelle, et non pas également celui d'Ouseramon, son fils, héritier et successeur.

Enfin, du point de vue du sens, si le nom du père d'Ouseramon est effectivement mentionné ici, pourquoi le serait-il à travers l'idée d'être affublé d'un grand/gros membre viril : « Ouseramon fils de "c'est considérable : (son) phallus"» ? Que signifierait cette allusion ? Serait-elle employée dans le but d'exalter la puissance d'Âamtjou, le vizir en titre ? Même au sein d'une élaboration littéraire, tout aussi singulière soit-elle, l'évocation de cette qualité et cette démonstration de force au moyen d'une telle tournure nous paraît quelque peut curieuse, d'autant plus qu'elle ne semble pas avoir de lien direct avec l'ensemble du texte. Au demeurant, si l'objectif avait effectivement été d'insister sur la puissance qu'il détenait grâce à sa fonction, nous nous attendrions alors à ce que son nom fût, en plus, précédé de son titre de vizir. Tant phonétiquement que sémantiquement, l'expression aurait eu bien plus d'impact auprès de l'auditoire ramesside si elle avait été la suivante : * « Ouseramon fils du vizir Âamtjou (i.e. "c'est considérable : (son) phallus") ${ }^{50}$.

Un autre problème, graphique cette fois, subsiste encore quant à la lecture de l'ensemble de cette phrase. En effet, Quack fait pertinemment remarquer que, dans sa compréhension du passage, Helck a omis la difficulté posée par le déterminatif de l'homme assis (Gardiner A1) accolé au mot qu'il considère comme étant $m \underline{t} 3$ " phallus, membre masculin ». Il ne s'agit pourtant pas du déterminatif habituel répertorié dans le Wörterbuch ni dans les autres dictionnaires et lexiques pour ce mot ${ }^{51}$, et en l'occurrence, la graphie de ce terme controversé a toute son importance au sein de ce débat. D'autre part, l'anthroponyme Âamtjou est-il attesté avec cette graphie particulière 
dans d'autres documents égyptiens ?

À notre connaissance, une seule autre occurrence du prénom Âamtjou présentant une graphie identique à notre séquence problématique est répertoriée sur la quatrième ligne du verso de l'ostracon BM 5672 + O. CGC 25649. Il s'agit d'un ouvrier de Deir al-Medineh ayant vécu sous Ramsès IX, donc postérieurement à la rédaction de notre papyrus ${ }^{52}$. Schneider différencie toutefois ce nom de personne de celui du vizir Âamtjou. En raison du déterminatif (D53), il pense qu'il existait probablement un surnom égyptien vulgaire ${ }^{3}-m-\underline{t} z$ 《 avec un gros phallus ». Du point de vue du sens, il rapproche également ce sobriquet avec d'autres expressions de plusieurs dialectes proche-orientaux appartenant au même champ sémantique ${ }^{53}$.

Concernant la présence du déterminatif inhabituel de l'homme assis (Gardiner A1) dans la graphie du mot $m \underline{t} 3$, le texte de l'ostracon CGC 25209 (période ramesside) est tout à fait intéressant à ce propos ${ }^{54}$. Il s'agit d'un hymne à Osiris dans lequel le dieu est qualifié de " momie au long phallus " ( $s$ h $3 w$ mtzs). Selon le texte hiératique et la transcription d'Erman, qui est la seule existante à ce jour, le mot $m \underline{t} 3$ est doublement déterminé, à la fois par le signe du phallus (Gardiner D53) ainsi que par celui du divin (dieu assis, Gardiner D40 ${ }^{55}$. Le même couple de déterminatifs est également présent dans le papyrus mythologique du Delta (début de l'époque saïte, vraisemblablement règne de Psammétique $\left.\mathrm{I}^{\mathrm{er}}\right)^{56}$. Ainsi, à l'instar de la graphie de $m \underline{t} z$ désignant le phallus divin d'Osiris, l'orthographe de $m \underline{t} 3$ dans notre papyrus pourrait dès lors déterminer le phallus humain d'Ouseramon $^{57}$. Du reste, il serait parfaitement cohérent que, au moyen de ce déterminatif de l'homme assis, une partie du corps humain - strictement masculine - soit catégorisée comme appartenant également, d'une manière plus générale, au grand ensemble « être humain masculin ${ }^{58}$.

Ainsi, l'anthroponyme Âamtjou, écrit de manière identique à celle consignée sur notre papyrus, pourrait effectivement être corroborée. Il s'agirait cependant du seul autre document attestant de cette orthographe inhabituelle pour le nom dont il est question $^{59}$. En revanche, graphiquement, rien ne s'oppose à ce que, dans notre texte, $m \underline{t} 3$ se réfère au phallus du futur vizir Ouseramon.

Signalons en dernier lieu une ultime possibilité. Le groupe $\varsigma_{3 . t}+m+c+\underline{t} 3+\mathrm{D} 53$ pourrait éventuellement être un schématogramme ayant une fonction purement phonétique afin d'écrire la séquence `3-mt et non « c'est considérable : (son) phallus ». Pour un scribe de l'époque ramesside, ce groupe solidaire aurait été une simple variante graphique afin d'écrire le nom Âamtjou, et cela sans aucune allusion vulgaire $^{60}$. De ce point de vue, une telle orthographe de cet anthroponyme n'aurait alors pas de véritable signification pseudo-étymologique.

En définitive, après avoir considéré l'ensemble des aspects, aucun argument qui ne laisserait place au doute et qui résoudrait définitivement ce problème ne semble émerger de la documentation égyptienne. En revanche, les différents faisceaux d'indices en faveur de la lecture littérale suivante : « $\left[\right.$ Mon coeur ${ }^{3}$ est grandement penché (vers) Ouseramon. Le phallus, combien d'années [jusqu'à maintenant depuis qu'il (= le phallus) a été défait ?] » et allant ainsi à l'encontre d'une acception tant pseudo-étymologique que purement phonétique du nom d'Âamtjou nous incline à privilégier la proposition déjà formulée par Helck ${ }^{61}$.

Rappelons, toutefois, que le problème repose avant tout sur la difficulté de lecture d'un seul signe partiellement en lacune ainsi que sur l'inhabituel déterminatif (A1) pour le terme $m \underline{t} 3$.

${ }^{f}$ : Helck transcrit $p 3 y=j n b \quad n f r$, probablement dû au fait que c'est une collocation fixe dans les textes ramessides $^{62}$. Sur la base de la planche de Rossi, Stauder proposait, éventuellement, une autre possibilité :

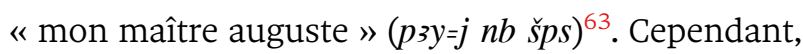
en acceptant cette lecture, il manquerait le second trait du signe šps. Dans ce cas, ce dernier est peutêtre ligaturé avec le $s$. Nous avons tout d'abord pensé à lire la séquence $p 3 y=j n b n t r n f r$ ( « mon maître le dieu parfait »), selon Möller II, 547 et 180. Néanmoins, étant donné, à notre connaissance, que l'expression standardisée $p$ sy $=j$ $n b \quad n f r$ n'est jamais complétée du signe $n t r$ (Gardiner R8), nous suivrons donc la formulation couramment attestée $p 3 y=j n b n f r$, bien que le signe équivoque ressemble davantage à $n \underline{t} r$ (Gardiner R8) qu'au faucon sur le pavois (Gardiner G7) ${ }^{64}$. $\mathbf{g}^{\mathbf{g}}$ : Le texte présent dans la lacune servait vraisemblablement à introduire le vizir Âamtjou, et peut-être même à présenter son discours, comme le pensait Helck $^{65}$, étant donné que le père d'Ouseramon n'a 
pas encore été mentionné. Cette hypothèse se trouve renforcée par le fait qu'il y a une mise en résonnance thématique entre :

- Thoutmosis III (= le roi actuel)/son père (= le roi précédent) ;

- et Ouseramon (= le vizir actuellement nommé)/ son père (= le vizir précédent).

Toutefois, il est également possible que ce soit le début du discours d'Ouseramon lui-même.

h : Litt. " On le trouve en tant qu'homme ». Remarquons ici que la construction glosante $m r r=f$ $p w$ (forme nominale imperfective passive) ne présente pas de gémination. C'est peut-être un indice grammatical de la datation de la rédaction du texte, puisqu’à l'époque ramesside, la distinction entre forme écrite courte et longue est devenue interchangeable. De plus, dans la construction ancienne, le $p w$ serait placé directement après $g m=t w=f^{66}$.

': Suite à la vérification du texte d'après l'image numérisée, nous confirmons et suivons ici la transcription déjà proposée par Černý ${ }^{67}$. Litt.: « Cela signifie qu'on le trouve comme un homme dont les yeux transpercent » (ou « dont les yeux sont perçants » ou " aux yeux perçants »).

Les transcriptions de ce passage publiées par Helck ${ }^{68}$ posent un problème paléographique et sémantique :

- Problème paléographique : ce n’est pas ḩjj (Möller II, 307) qui est inscrit sur le papyrus, ni sur la planche de Pleyte et Rossi. Černý a transcrit le signe de l'oeil fardé (Möller II, 83) sur le signe du bras armé (Möller II, 105) avec justesse. La lecture de Helck est donc à corriger. L’oeil fardé et le bras armé sont les déterminatifs du mot $\underline{d} d t$. C'est un terme, peu fréquent, en rapport avec la vue qui se traduit par " être aveuglé, être transpercé du regard, percer du regard $»^{69}$. L'expression $\underline{d} d t$ jr.t est attestée à plusieurs reprises dans la littérature ramesside et Basse-Époque :

- pap. Anastasi I, 11, 4 = pap. BM EA 10247 = Lettre satirique de Hori (fin XIX dynastie, règnes de Séthy II à Taousert) ${ }^{70}$;

- pap. Lansing, 3, 8 = pap. BM EA 9994, r ${ }^{\circ}$ 3, 8 (fin $\mathrm{XX}^{\mathrm{e}}$ dynastie $)^{71}$;

- pap. Bankes II, 4 = pap. BM EA 75017 = Lettre de Tjaroy (Thoutmosis) à Boutehamon (fin $\mathrm{XX}^{\mathrm{e}}$ dynastie, règne de Ramsès XI) ${ }^{72}$;

- Pap. Brooklyn 47.218.135, 5/17 = sagesse de
Basse-Époque (fin XXVI ${ }^{\mathrm{e}}$ dynastie, terminus post quem : règne d'Apriès) ${ }^{73}$.

- Problème sémantique : " c'est un homme dont on dit (de lui) les yeux apparaissent... " présente une difficulté puisque le verbe $h^{`} j$ se rapporte majoritairement à la figure royale et divine. Que voudrait donc dire cette proposition ? Est-ce une métaphore ? Helck, suivi par Dziobek, traduit : «On le trouve comme un homme dont on dit : ses yeux brillent », sans commenter ce passage. Certes, h`j peut également se traduire par « briller », mais, dans la mesure où le verbe $\underline{d} d t$ est en rapport avec la vue, le substantif $j$ r.t qui suit est tout à fait approprié.

j: Litt. « qui est rempli de paroles (/discours) » ${ }^{74}$. Le sens de toute cette phrase est probablement : "c'est un homme à l'intelligence (/perception) acérée (/perspicace) et aux discours élégants ».

${ }^{\mathbf{k}}$ : Restitution, très vraisemblable, proposée par Hel$\mathrm{ck}^{75}$, d'après le sens du début de la 1.7 (« [Ouseramon] ${ }^{7}$ est son nom en vérité »).

': Proposition de restitution littérale, d'après les commentaires de Helck et de Stauder ${ }^{76}$ : " On [se tourne] ${ }^{\mathbf{8}}$ vers (/sur) l'argent par manque (/rareté/ en l'absence de l'or) lorsque (/pendant que) le roi protège (/est occupé à protéger) [l'Égypte] ». Le jw est dans ce cas compris comme étant circonstanciel (voir infra § 3.3.3). Nous ne pouvons pas totalement exclure qu'il s'agisse de deux propositions distinctes, présentées sous la forme de deux proverbes juxtaposées. Le jw serait alors un indicateur d'énonciation « classique »: « Mais n'est-ce pas vers l'argent [qu'il faut se tourner] lorsque l'or est rare ? Le roi protège [l’Égypte ...] ».

Par ailleurs, comme le note avec justesse Dziobek, le signe $\underline{t} s$ transcrit par Helck est une erreur (Möller II, 535).

m : Restitution proposée par Helck et Černý à partir de l'ostracon CGC $25339^{77}$. Litt. : « [Tu t’approches du Maître du Double-Pays alors (/tandis) que l'Horus] est dans sa demeure, (y) étant souverain, et que (/ alors que) le roi est dans son palais ». Notons la mise en parallèle grammaticale, lexicale et sémantique des propositions [jw $H r] m p r=f$ et $j w n s w . t m\ulcorner h=f$.

${ }^{\mathrm{n}}$ : Proposition de restitution grâce à deux textes similaires présents dans la tombe du vizir Rekhmirê (TT 100 : [jnk] gr.t h.sty $n(y) n b$ c.w.s sdm.wy Hr jr.ty 
jty) ainsi que dans celle du héraut Antef (TT 155 : jr.ty nsw.t hasty $n b$ (h) $)^{78}$.

\section{Commentaires philologiques et his- toriques}

\subsection{Structure}

La structure du texte est organisée en plusieurs séquences :

- un ancrage temporel précis fourni par les deux premières lignes qui insistent par deux fois sur la date (« l'an 5 de Thoutmosis III » et « en ce jour»). C'est précisément ce jour, et pas un autre ;

- un ancrage thématique défini : une audience royale qui a pour objectif d'acter la nomination d'Ouseramon au poste de vizir. C'est cette occasion-là, et aucune autre, qui est développée ici ;

- un ancrage spatial défini : " au-devant de Sa Majesté » ;

- une série de dialogues et de discours : dialogue entre le roi et Ouseramon ( ?), puis discours, probablement, d'Âamtjou.

Cette structure et cette écriture ont pour objectif de mettre en scène la nomination d'Ouseramon, au moyen d'un préambule permettant de nous mettre en situation (ancrage temporel, spatial et thématique précisément définis) afin de contextualiser les dialogues et discours. Ce texte pourrait tout à fait être joué.

Le début du texte du papyrus de Turin (1. 1-2) reprend la structure habituelle des textes dits « d'introduction $»^{79}$ en présentant les éléments suivants :

- une date :

«An 5, 1er mois de la saison-akhet, le 1er jour, sous la majesté du roi de Haute et Basse-Égypte (Menkheperrê)| le fils de Rê (Thout[mosis)| v.s.f.] » H3.t-sp 5 зbd 1 3h.t sw 1 hr h̆m (ny) nsw.t-bjty (Mn$\left.h p r-R^{\Omega}\right) \mid s 3 R^{\complement}($ Dhwty- $[m s) \mid$ c.w.s.],

- le jour de l'introduction de $\mathrm{X}$ :

«En ce jour de l'introduction du scribe du sceau divin Ouseramon du domaine d'Amon » hrw pn (ny) bs sš httm-nțr Wsr-Jmn n(y) pr-Jmn,

- un lieu d' « introduction » : « au-devant de Sa Majesté » $m-b 3 h h m=f$,

- [un discours inaugural] : « [Sa Majesté a dit »] $[\underline{d} d \sim j n h m=f]^{80}$.

À noter que, dans les « textes d'introduction » de Karnak, le discours inaugural était prononcé par la personne introduite dans sa nouvelle fonction, et non par l'agent qui l'a nommé, c'est-à-dire dans le cas présent le roi. Nous avons donc ici une adaptation de ce genre de texte.

La partie « dialogues et discours » (1. 2-9) est, quant à elle, composée de trois temps. Tout d'abord, le roi s'adresse, à priori, très directement à Ouseramon. C'est un échange direct composé d'une question-réponse $(=\mathrm{T} 1 ; 1.2-5)^{81}$. S'en suit, vraisemblablement, un discours du vizir Âamtjou, père d'Ouseramon, adressé au roi. Ce propos est lui-même subdivisé en deux sous-temps : un discours durant lequel il vante les qualités de son fils et fait l'éloge de ses compétences auprès du roi $\left(=\mathrm{T} 2 \_\mathrm{A} ; 1.5-7\right)$, puis il décrit brièvement la position du vizir en général $\left(=\mathrm{T} 2 \_\mathrm{B}\right.$; 1. 8). Enfin, vraisemblablement toujours Âamtjou s'adresse cette fois directement à son fils, Ouseramon, afin de lui résumer l'essence même de la fonction de vizir tel un Enseignement, avec le roi comme troisième personne délocutive (= T $3 ; 1.8-9)$.

La séquence " dialogues et discours » parait donc fonctionner de manière triangulaire, comme l'illustre le schéma ci-contre (Fig. 7). Bien que le roi semble s'adresser tout d'abord directement à Ouseramon, il n'en reste pas moins que c'est, vraisemblablement, le vizir Âamtjou qui fait le lien entre le roi et son fils, le futur vizir.

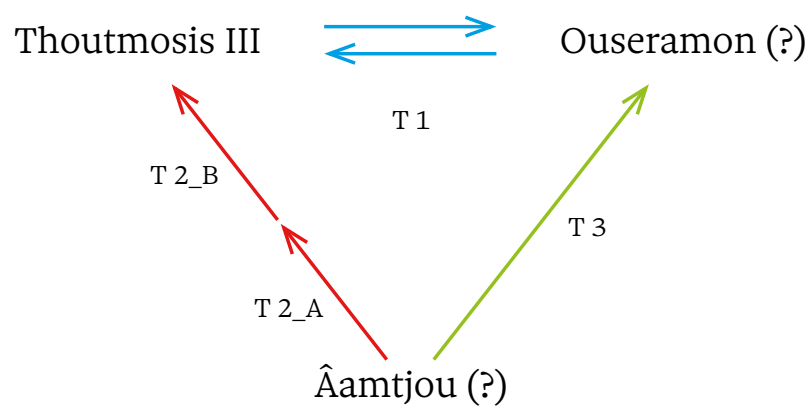

Fig. 7 : Schéma du fonctionnement triangulaire des échanges dans le pap. Turin Cat. 1878 vº, I. 2-9 (hypothèse $n^{\circ}$ 1). Schéma de l'auteur.

Cette modélisation schématique est plausible et cohérente. Elle dépend néanmoins essentiellement de deux facteurs. D'une part, en l'état actuel nous 
ne connaissons pas de manière certaine l'identité de l'individu répondant à la question posée par le roi (1. 3-5). D'autre part, la restitution du nom Âamtjou dans la lacune à la fin de la ligne 5 reste une hypothèse.

La possibilité qu'il y ait eu initialement le nom d'Ouseramon, et non celui de son père, doit donc être envisagée, de même que le fait que ce soit Âamtjou qui réponde à la question (Fig. 8). La phrase de la troisième séquence $(=\mathrm{T} 3 ; 1.8-9)$ présentant un changement d'adressivité (« tu es » au lieu de « on le considère », « [c'est quelqu'un] qui »), il s'avèrerait logique qu'elle soit adressée à Ouseramon par son père et précédesseur Âamtjou, comme nous l'envisageons dans l'hypothèse $n^{\circ} 1$. Là encore le schéma met en exergue un fonctionnement triangulaire des échanges.

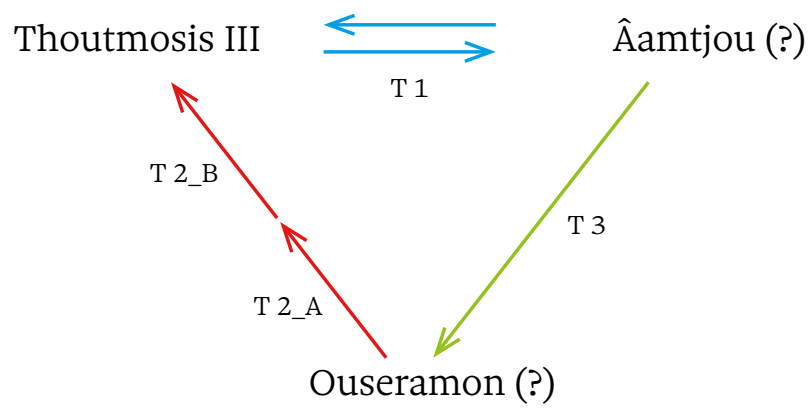

Fig. 8 : Schéma du fonctionnement triangulaire des échanges dans le pap. Turin Cat. 1878 Vº I. 2-9 (hypothèse $n^{\circ} 2$ ). Schéma de l'auteur.

Cette deuxième modélisation présente cependant un problème majeur du point de vue du sens. En effet, Ouseramon parlerait de lui à la troisième personne, ce qui paraît quelque peu étrange, à moins qu'en réalité l'intégralité de cette partie (T 2_A et B) soit un genre de proverbe et non pas un discours. De plus, Âamtjou ne serait plus l'élément central effectuant le lien entre le souverain et son fils et successeur direct.

Ainsi, l'hypothèse $\mathrm{n}^{\circ} 1$ semble mieux fonctionner, tant sur le plan de la cohérence de l'enchaînement de l'identité des locuteurs, de la dynamique d'adressivité de leurs discours que sur celui du contenu de leurs échanges. Il va de soi que notre inflexion vers la première proposition du fonctionnement de la conversation reste soumise à l'état fragmentaire actuel du texte.

La scène de la Nomination, gravée puis peinte, dans la tombe d'Ouseramon (TT 131) illustre quant à elle parfaitement le fonctionnement triangulaire des échanges, tel qu'envisagé dans la modélisation schématique $n^{\circ} 1$ (Fig. 9). Elle est située dans la salle transversale de la tombe, c'est-à-dire dans l'espace d'auto-présentation du défunt, sur la partie nord du mur est (= partie droite en entrant). Il s'agit du même événement que celui décrit dans notre papyrus, c'est-à-dire l'audience royale concernant la nomination d'Ouseramon dans la fonction de vizir. Une assemblée de dignitaires, avec à leur tête le vizir Âamtjou vieillissant, va à la rencontre du roi Thoutmosis III, représenté assis sous son kiosque, afin d'obtenir un "bâton de vieillesse ", c'est-à-dire un successeur. Ouseramon est en fin de cortège. Comme dans le texte du papyrus, Âamtjou est l'intermédiaire entre le roi et son fils dans cette passation de fonction. Alors encore vizir en titre, il est représenté selon l'iconographie canonique de sa fonction depuis la XIII dynastie - un long vêtement complété par un collier dont l'accroche, placée à la base de la nuque, est en forme de cartouche - alors que son fils est vêtu d'un simple pagne. Le choix de cette iconographie concorde avec le fait qu'il se désigne par le titre "scribe du sceau divin » (š̌ htm$n \underline{t} r)$, indiquant ainsi le fait que le processus de sa nomination n'est pas encore terminé.

Par ailleurs, la comparaison avec la structuration du deuxième discours du roi dans la Nomination (TT 131, col. 25-34) renforce notre hypothèse du fonctionnement des échanges dans notre texte. Le roi s'adresse tout d'abord directement à Âamtjou (col. 25 = T 1). Il lui parle ensuite de son fils Ouseramon en reprenant la formulation et les thèmes des qualités idéales du candidat recherché (col. 26 = $\mathrm{T}$ 2). Enfin, Thoutmosis III dicte des injonctions à Âamtjou au sujet de l'exercice du vizirat $(=\mathrm{T}$ 3_A afin que celui-ci les transmette à son fils (= T 3_B). Le discours royal devient même parfois totalement métaphorique (col. 27-34). La modélisation schématique de cette séquence met à nouveau en évidence une dynamique triangulaire dans laquelle Âamtjou est indéniablement le relai entre le monarque et son fils (Fig. 10). 


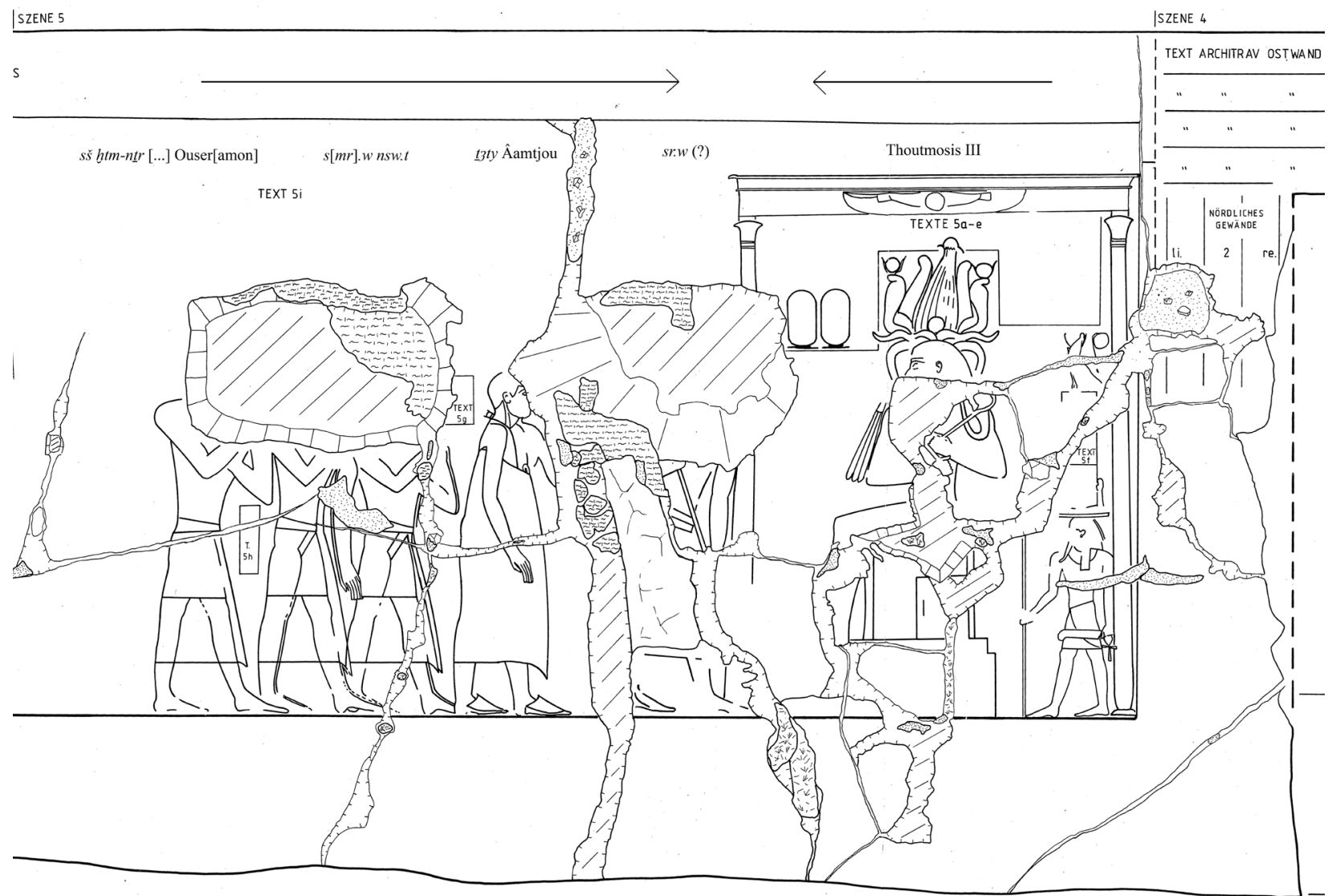

TT 13r, Querhalle Ostwand Nord, Szene 131-4, 5-7. I: I5, S. 64, 7rff.

Fig. 9 : Scène de la Nomination du vizir, tombe d'Ouseramon (TT 131). Tiré de Dziobek, Gräber Vezirs User-Amun, 1994, pl. 72 [scène 131-5].

Thoutmosis III

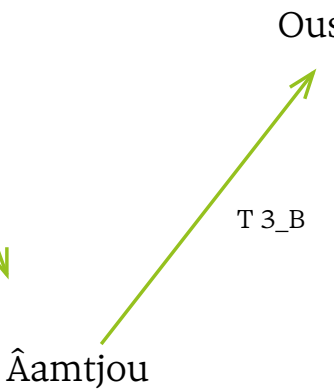

Fig. 10 : Schéma du fonctionnement triangulaire des échanges dans la Nomination (TT 131, col. 25-34: $2^{\text {e discours }}$ du roi). Schéma de l'auteur.

L'élaboration littéraire du papyrus de Turin semble donc avoir repris, tout en l'adaptant, la structuration du discours royal gravé dans la tombe d'Ouseramon (Figs. 7, Fig. 910). La parole royale ne descend plus vers Âamtjou, c'est au contraire ce dernier qui conseille le souverain en louant les qualités et les compétences de son fils. Il ne s'agit donc plus d'une eulogie d'Âamtjou et d'Ouseramon formulées à la deuxième personne par l'intermédiaire de la bouche du roi, mais d'une eulogie d'Ouseramon énoncée, à la troisième (1. 5-8) puis à la deuxième personne (1. 8-9), par son père et prédécesseur.

\subsection{Langue et langage}

Le texte du papyrus Turin Cat. 1878 v $^{0}, 1.1-9$ est rédigé en moyen égyptien avec des particularités néo-égyptiennes, caractéristiques de l'aspect hybride de la littérature à partir du règne d'Amenhotep IV $^{82}$. Citons, par exemple, l'emploi de pronoms possessifs néo-égyptiens $\left(1.4: p_{3} y=j n b\right.$ et $1.5: p 3 y=k$ $j t)$, la construction du nombre cardinal (1. $4: 30 n(y)$ rnp.t t3y), l'expression du pluriel néo-égyptien au moyen de l'article défini (1. $4: n 3[n(y) r n p . w t])$, l'emploi de l'adverbe $g r$ après le syntagme sur lequel il porte $(1.5: j r\{r\}=j t 3 j \text { jw.t } n \text { p } 3 y=k j t g r)^{83}$, l'utilisation de l'article défini féminin singulier placé devant le substantif qu'il détermine (1. $\left.5: t_{3} j 3 w . t\right)$, et enfin la collocation ramesside $p 3 y=j n b n f r(1.4)$. 
Restitutions prises en compte, nous dénombrons actuellement un total de 155 mots pour 86 occurrences uniques. La quantité de même que la diversité des termes employés témoignent nettement de la prédominance du roi (un total de 20 occurrences pour 14 entrées uniques). De plus, le souverain agit à plusieurs reprises : au moyen d'une déclaration assertive il « [dit] » ([d $d d])$ qu'il « approuve grandement " ([jb] $h n$ ( $r) \mathrm{N} r$ - $\left.{ }_{3} . t\right)$ Ouseramon, puis il pose une question directe, et enfin il " protège [l'Égypte]» $(h w[K m . t])$. Par ailleurs, le monarque est précisément localisé : la scène se déroule « au-devant " de sa personne ( $m-b 3 h h h m=f)$, il est « dans sa demeure » $(m p r=f)$ et « dans son palais » $(m \stackrel{c}{h=f})$. Il est aussi le garant de l'intégrité de « [l’Égypte] » ([Km.t]).

Le focus se déporte ensuite sur la figure du vizir, incarné ici au travers des personnages historiques d'Âamtjou et d'Ouseramon. Le vizir est « un homme " $(s)$ dont les qualités sont d'avoir le « regard perçant » (d $d d t j r . t)$ et des « discours élégants » $(m h w m h n . w)^{84}$. Intrinsèquement, il est le « coeur [du maître] » (hsty $[n(y) n b])$ ainsi que « [les (deux) oreilles d'Horus et les (deux) yeux du souverain] " ([sdm.wy Hr jr.ty jty]). C'est à ce titre qu'il est habilité à « [s'approcher du Maître du Double-Pays] » ([hnhhn $n b \quad t 3 . w y])$. En termes d'action, il accorde ( $r d j)$ sans cesse le métal précieux qu'est l'argent (hd $\underline{d})$. Enfin, c'est quelqu'un qui a précédemment exercé ( $j r j)$ des «fonctions » $(j 3 w . t)$ telles que « scribe du sceau divin du domaine d'Amon » (š̌ httm-ntr $n$ n(y) pr-Jmn), avant de recevoir une « initiation » (bs) actant sa nomination et le rendant ainsi apte à exercer entièrement et de plein droit le vizirat (pour ce dernier point en particulier voir infra $\S 3.3 .1$ ).

En dernier lieu, notons que le roi et le vizir sont mis en parallèle au travers de la métaphore de l'or et de l'argent (nbw et $h \underline{d} \underline{\text { ) }}$ (voir infra $\S 3.3 .3$ ).

\subsection{Contenu}

Le contenu du texte de notre papyrus est assez énigmatique. D'un point de vue général, le document rapporte la nomination d'Ouseramon à la fonction de vizir en l'an 5 de Thoutmosis III. De manière plus détaillée, plusieurs éléments méritent d'être commentés afin d'en saisir toute la teneur.

\subsubsection{La nomination du vizir Ouseramon}

Deux attestations de la nomination d'Ouseramon nous sont connues : l'une représentée dans sa tombe et accompagnée de textes en hiéroglyphes (TT 131 - Nomination du vizir) ${ }^{85}$, et l'autre inscrite en hiératique sur le verso du papyrus Turin Cat. 1878. Helck a été le premier à avoir compris la signification du texte relaté sur le papyrus de Turin et à l'avoir mis en relation avec le texte de la Nomination d'Ousera$m_{0}{ }^{86}$, puisque le texte consigné sur le papyrus relate le même événement.

Les deux documents sont naturellement de longueurs différentes. La scène de la Nomination dans son ensemble occupe la moitié de la longueur du mur, soit environ $2,50 \mathrm{~m}$ de long sur $2,40 \mathrm{~m}$ de hauteur. Le texte hiéroglyphique s'étend sur 36 colonnes, avec une hauteur de quadrat d'environ $7 \mathrm{~cm}$. Dans l'état actuel de conservation, c'est le texte le plus long et le plus étendu de la tombe d'Ouseramon. Pour sa part, le texte hiératique du papyrus se développe sur 9 lignes fragmentaires.

Dans ces deux documents, le cadre général, l'évènement unique de nomination et les acteurs sont identiques. La Nomination est une des rares Königsnovelle en contexte privé, dont l'objectif est certes d'afficher la grande distinction qu'Ouseramon reçoit de la part du roi, mais surtout de mettre en scène la performativité de la parole royale dans sa propre tombe ${ }^{87}$. Le papyrus de Turin n'est en outre pas le modèle hiératique ayant inspiré le texte de la tombe, ni un résumé de ce dernier. C'est un texte indépendant puisque le contenu et la forme stricto sensu sont différents, et seul le papyrus apporte la précision de la date de l'an 5 de Thoutmosis III. De par sa structure et son aspect littéraire, le papyrus de Turin n'est donc pas un document administratif, mais une élaboration littéraire ramesside (voir infra § 5).

Que ce soit dans le contexte d'auto-présentation de la tombe, ou dans celui d'une production littéraire sur papyrus, la promotion d'Ouseramon au poste de vizir s'effectue à titre individuel et est présentée comme étant méritocratique - ce qu'elle n'est bien sûr pas uniquement dans la réalité. La même insistance sur la continuité père/fils est également présente dans la phraséologie des deux textes avec par exemple : 
" déjà sous ton père j'ai exercé la fonction "

$j r\{r\}=j t 3 j 3 w . t n p z y=k j t$ gr (Pap. Turin Cat.

$\left.1878 \mathrm{v}^{0}, 1.5\right)$

« Tu as bien installé son fils dont le nom est

Ouser comme scribe du sceau divin dans le temple d'Amon. Son père était déjà dans l'entourage de ton (grand)-père le roi de Haute et Basse-Égypte (Âa-kheper-ka-Rê)|, justifié »

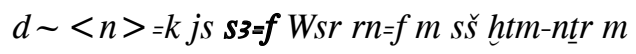
hw.t-ntr $n(y . t) J m n .[W] n$ jt=f $m h(3) w j t=k n s w . t-$ bjty (`3-hpr-k3-R`)| m3 ${ }^{\complement}-[h r] w$ (TT 131, Nomination, col. $24,2^{\mathrm{e}}$ discours des courtisans)

Celle-ci est aussi renforcée par l'iconographie de la scène et la structure du texte issu du papyrus dans lesquelles Âamtjou fait le lien entre son fils et le roi afin d'assurer la transmission de la fonction à sa descendance.

Les bribes du discours d'Âamtjou dans le papyrus de Turin ne sont pas sans rappeler certaines des formulations présentes dans les autres textes du cycle viziral :

l'Enseignement d'Âamtjou (TT 131) ${ }^{88}$, l'Installation $d u$ vizir (TT 131, TT 100, TT 66) ${ }^{89}$, les Devoirs du vizir (TT 131, TT 100, TT 29 et ostraca MANT, TT 106) ${ }^{90}$, le Bureau du vizir (TT 100) ${ }^{91}$, l'Autobiographie de Rekhmirê (TT 100) ${ }^{92}$, et surtout la Nomination $d u$ vizir (TT 131) qui fait directement écho à notre texte, comme nous l'avons souligné.

La stèle conservée au musée de Grenoble fait également référence, dans une moindre mesure, à la nomination d'Ouseramon au poste de vizir :

«(Mon) excellence ayant promu (ma) position, j'ai été initié à cette excellente fonction de directeur de la Ville et vizir » $s h n t \sim n j q r(=j) s . t(=j) b s=k w r j 3 . t$ tn mnh.t $r$ jmyr3 njw.t tzsty (Stèle Grenoble MG 1954,1.7) ) $^{93}$

Comme dans la deuxième ligne de notre papyrus, c'est le verbe $b s(j)$ qui est employé pour désigner cette nomination. L'initiation des substituts du pharaon, c'est-à-dire des prêtres et des vizirs, est exprimée au moyen de ce verbe, notamment dans ce qui est appelé « textes d'introduction » ainsi que dans leurs biographies. Ce terme porte en effet le sens d'une véritable " initiation ». Comme l'a très justement exposé et commenté Kruchten, c'est un verbe de mouvement, dont la signification primaire est « jaillir » au sens de « monter ». $B s(j)$ indique également une transformation, c'est-à-dire un changement d'état et/ou de statut autant que le passage d'un monde à un autre, notamment du monde profane au monde sacré ${ }^{94}$. Le sens de ce verbe affirme le droit désormais acquis de l'officiant qui a été initié d'exercer sa fonction ou de célébrer les rites auxquels il a été instruit.

Par ailleurs, dans le papyrus de Turin, l'expression - bien que partiellement lacunaire et donc hypothétiquement restituée - $[j b=j] h n(r / n)$ Wsr-Jmn $r$ - $3 . t$ exprime le fait que le roi s'appuie grandement sur les compétences d'Ouseramon. Le verbe $h n / h n n /$ hзn signifie littéralement " s'incliner, (se) pencher, (se) courber, approuver, avoir confiance, s'appuyer sur ${ }^{95}$. Dans les procédures oraculaires, ce terme est employé afin d'indiquer la réponse favorable de la part d'un dieu. Il se rapporte au geste effectué par la statue divine portée par les prêtres lors de la proces$\operatorname{sion}^{96}$. En outre, dans la biographie du grand-prêtre d'Amon Româ Roÿ préservée sur l'une de ses statues (XIXe dynastie, règnes de Ramsès II à Sethy II), le terme possède l'acception « s'en remettre à, s'appuyer sur $»^{97}$.

Ainsi cette tournure littéraire exprime et explicite, selon nous, le fait que Thoutmosis III est précisément en train de le nommer au poste de vizir. Dans ce cas, par extrapolation, la proposition pourrait être comprise d'une manière plus directe : " je (= le roi) nomme Ouseramon ». Cette formulation serait donc à ajouter à la longue liste des termes et expressions en relation avec la nomination et la promotion d'un individu existants dans l'Égypte antique. Černý semble lui aussi avoir compris ce passage dans ce même sens puisqu'il translittère [d] $h n$ en renvoyant à l'entrée correspondante dans le Wörterbuch, peut-être par rapprochement avec la version du texte de l'Installation présente dans la tombe du vizir Rekhmirê :

" Introduction ${ }^{2}$ ' du conseil dans la salle à colonnes de Pharaon, v.s.f. et faire que soit amené ${ }^{3}$ le vizir [Rekhmirê] nouvellement nommé »

st $3^{2}$ qnb.t $r$ wzhy $n(y)$ Pr-ç c.w.s. rd.t msw ${ }^{3}$ tsty $[R h-m j-R\urcorner d h n . w$ m mswt 
(TT 110, Installation, col. 1'-3' ' $^{98}$

Ce passage dans les versions des tombes des vizirs Ouseramon (TT 131) et Hapou (TT 66) est malheureusement en lacune, mais devait sans nul doute y figurer ${ }^{99}$.

Une autre mise en scène de nomination quelque peu postérieure à celle d'Ouseramon fait directement écho à cette dernière. Il s'agit de celle de Qenamon, l'intendant du port de Perou-nefer dans le Delta, en exercice durant le règne d'Amenhotep II. Sa sépulture se situe elle-aussi dans la nécropole thébaine de Cheikh Abd el-Gournah (TT 93), à quelques centaines de mètres à l'ouest de la TT 131 et surtout juste au-dessus de celle de son vizir contemporain Aménémopé (TT 29) ${ }^{100}$. Au sein de ce texte extrêmement fragmentaire, l'expression employée par le roi pour nommer Qenamon est $d h n$ (col. $7: h m=j r d . t$ $d h n[\ldots])$.

\subsubsection{Ouseramon et la pratique de la circoncision}

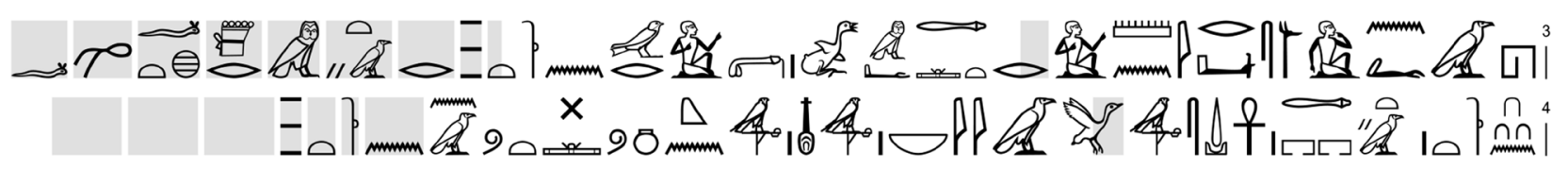

— " (...) Mt $\underline{3}$ wr $n$ rnp[.wt r t3y $m-\underline{d} r$ fh.t=f ?]

- $30 n(y)$ rnp.t tзy Pr-c3 c.w.s. pзy=j nb nfr. _ « (...) Le phallus, [depuis] combien de temps [a-t-il été défait ?]

_ ${ }^{4}$ Cela fait 30 ans, ô Pharaon v.s.f. mon maître parfait. (...)»

(Papyrus Turin Cat. 1878 v ${ }^{\circ}, 1.3-4$, extrait)

C'est à nouveau Helck qui propose la restitution de la fin de la ligne 3, sur la base du rapprochement du passage de notre texte avec l'inscription de la tombe de Khnoumhotep II à Beni Hassan (XII ${ }^{e}$ dynastie - Amenemhat II, Sésostris II et Sésostris III) ainsi que celle du rouleau de cuir de Berlin (milieu XVIII ${ }^{e}$ dynastie). Khnoumhotep parle de son père en ces termes :

« Il a gouverné sa ville, comme enfant, avant qu'il n’ait été défait du prépuce »

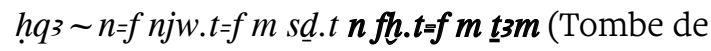
Khnoumhotep II, biographie, col. 184-186) $)^{101}$

Dans le rouleau de cuir, Sésostris $I^{\text {er }}$ dit à propos du dieu Rê-Horakhty :

« Il m’a promu comme maître des deux parties, comme enfant, avant que n'ait été délié pour moi le phallus » swsh $\sim n=f$ wj $r$ nb psš.t $2 \mathrm{~m}$ nhn $\boldsymbol{n}$ fh.t $n=\mathbf{j} \mathbf{m t z}$ (Rouleau de cuir de Berlin, Pap. Berlin 3029, I,9-10) ${ }^{102}$

Une autre attestation de cette expression est également à ajouter à ce dossier. La stèle Florence 2540, provenant du temple nord de la forteresse de Bouhen (XII ${ }^{\mathrm{e}}$ dynastie - Sésostris $\mathrm{I}^{\mathrm{er}}$ ), est un panégyrique royal vantant les qualités et les grandes actions qu'a réalisées le roi à une période pendant laquelle :

« il n’avait pas encore été défait de son prépuce »

$m$ nhn $\boldsymbol{n}$ fh.t $\mathbf{f} \boldsymbol{m} \mathbf{m} \mathbf{z} \boldsymbol{m}$ (Stèle Florence 2540, texte central, 1.6)

Selon Helck, le sens de la question-réponse du papyrus de Turin doit se comprendre ainsi : le roi demande à Ouseramon le temps écoulé depuis qu'il a été circoncis, et non depuis sa naissance. Ouseramon lui répond que cela fait 30 ans. Cette interprétation est celle retenue depuis ${ }^{104}$. Est-ce une tournure particulière pour s'enquérir de l'âge d'un individu ? Ou bien cet acte marque-t-il autre chose ?

La circoncision en Égypte antique est attestée tout au long de la période, et ce grâce à plusieurs types de sources provenant de différents contextes archéologiques : des restes humains momifiés, des statues, 
des documents iconographiques et textuels issus de parois de tombes et de temples, des stèles ou encore des papyrus. Nous constatons également une certaine diversité dans les termes et expressions employés afin de désigner cette pratique, de même que pour nommer l'absence de circoncision $\left(s^{e} b / s b . t\right.$, $\check{s}^{e} d$,

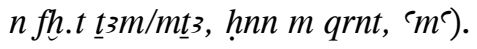

Le but de cette pratique ne semble pas être clairement explicité dans les sources égyptiennes et elle a suscité de nombreux débats dans la littérature égyptologique. Les données ne permettent pas de conclure qu'elle était pratiquée de façon régulière pour l'ensemble de la population masculine durant toute la période pharaonique. De ce fait, la circoncision ne semble pas correspondre à un rite obligatoire de passage à l'âge adulte. Les deux autres principales raisons avancées afin d'expliquer cet usage sont d'ordre médical et religieux (dans le cadre des thématiques de la naissance divine royale et des initiations rituelles), les deux n'étant, au demeurant, pas antinomiques. Lâge auquel cet acte était effectué reste difficile à définir avec précision et la ma- jorité des chercheurs le situe entre 10 et 15 ans $^{105}$. D'après la tournure de l'échange dans notre papyrus, la raison médicale semble, à priori, à exclure. La circoncision d'Ouseramon serait alors la marque de l'accession d'un individu à un statut particulier réservé aux initiés. La circoncision ne serait donc pas ici le résultat d'un traitement médical, ni un marqueur temporel, mais un marqueur social et statutaire.

De ce fait, le roi serait en train de demander à Ouseramon depuis quand il a été initié, sous-entendu peut-être à la prêtrise, et non de lui demander depuis quand il est adulte. Ouseramon lui répond que cela fait 30 ans, et précise qu'il exerçait déjà la fonction de [...] sous le père de Thoutmosis III, la fonction en question étant malheureusement en lacune dans le texte. En prenant en compte le fait que ce laps de temps est considérable en Égypte ancienne, il possède donc une grande expertise en matière de culte et des affaires divines - la quantité et la diversité des fonctions exercées au sein du domaine d'Amon vont dans ce sens ${ }^{106}$ - ainsi qu'une maturité suffisante afin de mener à bien sa future fonction de vizir.

\subsubsection{Comparaison métaphorique : l'argent et l'or pour le vizir et le roi ?}

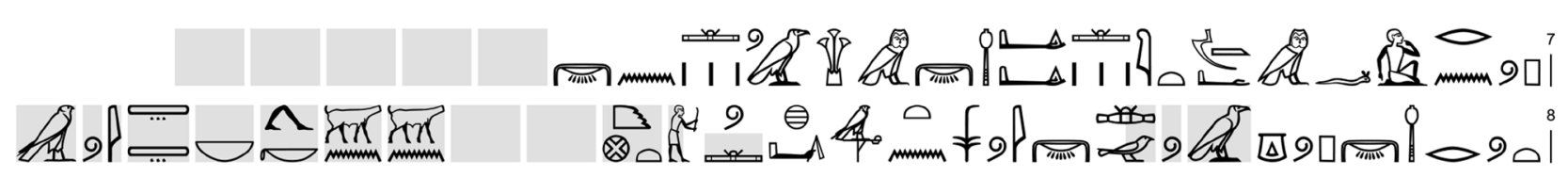

[Wsr-Jmn] ${ }^{7} p w r n=f m m_{3}{ }^{r} . t . \operatorname{Dd}(w) h \underline{h} m \underline{h} s w$ $n(y) n b w[\ldots]^{\mathbf{8}}=t w r h \underline{d} p w<m>g 3 w n(y) n b w$ jw nsw.t (hr) hww [Km.t].

[Ouseramon] ${ }^{7}$ est son nom en vérité. (C'est quelqu'un) qui accorde constamment de l'argent en excès par rapport à (/en plus de) l'or [...]. [C'est] ${ }^{\mathbf{8}}$ vers l'argent qu'il faut [se tourner] quand l'or est rare (/manquant) lorsque le roi protège [l’Égypte].

(Papyrus Turin Cat. 1878 v ${ }^{\circ}$ 1. 7-8)

La signification de la ligne 7 paraît, de prime abord, assez difficile à saisir. Helck note que la présence du nom Ouser devait certainement créer un effet de contraste entre la signification du mot wsr « (être) riche » (par opposition à « (être) pauvre »). Cette emphase continue directement dans la phrase suivante où il est précisé que le vizir, et dans le cas présent Ouseramon, dispense des richesses de manière abondante puisqu'il ajoute de l'argent à l'or, ou ajoute de l'argent en plus de l'or. La notion de richesse du vizir semble donc être mise en exergue ${ }^{107}$.

La ligne 8 est tout aussi problématique. Au-delà des difficultés de lecture et de compréhension dues aux lacunes, le contenu de ce passage est assez énigmatique. Helck propose de le comprendre ainsi : l'or est la métaphore du roi et l'argent celle du vizir. Labsence de l'or signifierait que le roi est en dehors du pays, en campagne militaire en train de protéger les frontières de l'Égypte, comme l'indique d'ailleurs le verbe $h w$ « protéger, garantir » et donc la restitution 
logique $h w[k m . t \text { ou } t 3 . w y]^{108}$. Cette protection- $h w$ semble exprimer l'idée d'immunité, mais ce terme peut avoir un caractère plus « offensif » que « défensif » lorsqu'il se trouve dans des contextes d'emploi se rapportant à des confrontations à l'étranger, et non en Égypte ${ }^{109}$. Cela pourrait être effectivement le cas ici, si cette interprétation devait être confirmée. La phrase est orientée vers un roi en train de guerroyer à l'étranger dans le but d'assurer la protection voire d'étendre son territoire. Dans ce cas, le vizir se substitue à lui afin d'assurer le bon fonctionnement et la bonne gestion du Double-Pays. Ainsi, la restitution du verbe en lacune à la fin de la ligne 7 pourrait vraisemblablement être « se tourner vers ».

L'assimilation métaphorique d'une divinité ou du roi à l'or est extrêmement courante dans les textes égyptiens ${ }^{110}$. La stèle de la tempête d’Âhmosis rapporte que :

« Sa Majesté a atteint le coeur de Thèbes, (c'est) l'or (qui) vient à la rencontre de [l'or] de cette image processionnelle » spr pw jr n hmm=fr $r$ hn W3s.t nbw hos $\boldsymbol{m}$ [nbw] sšm pn (Stèle de la tempête, $\left.\mathrm{r}^{\mathrm{o}} 12=\mathrm{v}^{\mathrm{o}} 14\right)^{111}$

À partir de la XVIII ${ }^{e}$ dynastie, le roi est fréquemment affublé de l'épithète « montagne d'or, électrum des rois $»^{112}$. Ainsi Amenhotep III est :

« le dieu parfait, montagne d'or » $n \underline{t} r n f r \underline{d} \boldsymbol{w} n(y) n b w$ (Temple de Soleb, embrasures nord et sud de la porte centrale IV-V) $)^{113}$ « le dieu parfait, montagne d’or, électrum des rois »

$n \underline{t r} n f r \underline{d} \boldsymbol{w} n(y)$ nbw $\underline{d}^{c} m n(y)$ nsw. wt (Temple de Louqsor, pièces sud, une des colonnes de la salle hypostyle) $)^{114}$

« le dieu parfait, montagne d'or » $n \underline{t r} n f r \underline{d} w n(y) n b w$ (Stèle et fragment de statue $)^{115}$

Ramsès II est lui aussi qualifié de :

« dieu parfait, montagne d'or, électrum de chaque roi » $n \underline{t r} n f r \underline{d} w \boldsymbol{n}(y)$ nbw $\underline{d}^{\top} m$ n(y) nsw.t $n b$ (Tanis, une des colonnes du « Temple de l'Est ») ${ }^{116}$
Pareillement, Ramsès IV, usurpé par Ramsès VI, est :

« le dieu parfait, montagne d'or, électrum des rois »

$n \underline{t} r n f r \underline{d} \boldsymbol{w} n(y) n b w \underline{d}{ }^{`} m n(y) n s w . w t$ (Temple de Medinet Habou) $)^{117}$

Terminons cette courte liste d'exemples avec celui d'Hatchepsout qui est qualifiée :

d' « électrum de la royauté »

$\underline{d} \boldsymbol{d} \boldsymbol{m} \boldsymbol{n}(\boldsymbol{y}) \boldsymbol{n s} . \boldsymbol{y t}$ (Grand temple de Karnak, obé-

lisque nord, soubassement sud, 1. 5) $)^{118}$

Dans le champ lexical de la métaphore aurifère, les caractéristiques attribuées aux êtres divins et royaux le sont aussi quelquefois aux hauts fonctionnaires reconnus et appréciés du souverain pour leurs qualités. Étant donné qu'il est aimé du roi, le chancelier, grand intendant du domaine royal et intendant du Double grenier Senneferi (XVIII ${ }^{\mathrm{e}}$ dynastie, Hatchepsout/Thoutmosis III), est :

« d'or et de lapis-lazuli »

$m r . t=f \boldsymbol{m} \boldsymbol{n b w}$ hr h $\mathrm{s} b d$

(Statues BM 48 et CGC 1013) $)^{119}$

De même, Aÿ et sa femme Tiy, richement récompensés et ornés de colliers d'or par le roi :

" ont été faits “personnes d'or" "

sy jry $m \boldsymbol{r m \underline { t }} n(\boldsymbol{y}) \mathrm{nbw}$

(Tell el-Amarna, TA 25) $)^{120}$

Bien que ce qualificatif n'a rien de surprenant puisqu'ils sont littéralement recouverts de colliers d'or, il y a là encore un lien entre ce qualificatif et le mérite de ces dignitaires.

En revanche, une telle assimilation cette fois-ci à l'argent semble plus rare, et nous possédons moins d'informations, de prime abord, sur la symbolique de l'argent en Égypte ancienne ${ }^{121}$.

Une connexion existe entre les os des dieux, des rois et des défunts et ce métal. Par exemple, les os de Rê :

« étaient d'argent, ses membres étaient d'or et ses cheveux étaient en lapis-lazuli véri- 
table »

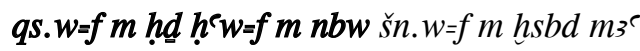

(Livre de la Vache Céleste 5-7) ${ }^{122}$

Dans le papyrus Jumilhac il est dit :

« l'or ce sont ses chairs et l'argent ce sont ses OS »

\section{$n b w$ jwf $p w$ hִd $q s . w=f p w$}

(Papyrus Jumilhac XIII,1) $^{123}$

Il en est de même dans les lamentations d'Isis et Nephthys dans lesquelles il est dit à propos d'Osiris :

« tes os sont fondus en argent »

(Papyrus Bremner-Rhind I,15,25) ${ }^{124}$

Enfin, deux des versions de la stèle du premier mariage hittite rapportent que les chairs de Ramsès II :

« sont en or, ses os sont en argent et tous ses membres sont en fer »

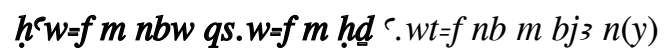

p.t (Stèle du premier mariage hittite, versions d’Abou Simbel et d'Éléphantine) ${ }^{125}$

Du point de vue religieux et symbolique, le couple or/argent, souvent indissociable, permet la cohésion des éléments par leurs propriétés minéralogiques respectives $^{126}$ et leur complémentarité. De même que l'argent est le métal qui représente l'ossature, structure invisible mais indispensable, permettant d'assurer le maintien, la mise en mouvement et la solidité du corps, le vizir est l'adjoint indispensable, qui assure la cohésion, la bonne gestion et le bon fonctionnement du pays, et ce d'autant plus lorsque le roi est occupé hors d'Égypte à garantir la protection des frontières du pays.

Indépendamment de ce système d'analogies or/ argent $=$ chairs $/$ os $=$ roi $/$ vizir $=$ visible $/$ invisible , Aufrère rappelle, dans son Univers minéral, que l'argent est associé à la pureté et qu'il constitue, dans l'architecture, le matériau privilégié des points de contact, c'est-à-dire par exemple les bases de colonnes et les architraves. Ainsi, lorsqu'il a rénové le portique du IV pylône de Karnak, Chabaka (XXVe dynastie) a refait :
« un grand portique plaqué d'or pur, les deux colonnes étant travaillées d'électrum et les deux bases les supportant d'argent pur » h3y.t ' 3.t mktj $m$ nbw nfr wh3.wy b3kw $m \underline{d}^{`} m$ sp.ty $\underline{h} \boldsymbol{r} . \boldsymbol{w} \boldsymbol{m} \boldsymbol{h} \underline{\boldsymbol{d}} \boldsymbol{w} \boldsymbol{w} \boldsymbol{b}$ (Grand temple de Karnak, porte du IVe pylône) ${ }^{127}$

Aufrère mentionne, par ailleurs, que l'argent est employé pour les parties de certains objets du mobilier funéraire de Toutânkhamon destinées à être en contact avec le sol. Il est apposé sur les poignées du sarcophage, sur les poignées de l'une des grandes chapelles, sur les patins du traîneau d'une petite chapelle ainsi que sur les poignées du traineau portant la boîte aux vases canopes ${ }^{128}$.

Largent apparait donc comme étant un matériau intermédiaire entre deux mondes destiné à marquer et à préserver la pureté de la partie sacrée qui est derrière la zone de contact. Ainsi, comme l'argent est le métal protecteur isolant le sacré pur du profane impur, le vizir assure le rôle d'intermédiaire protecteur entre le pharaon et le peuple.

Un autre exemple vient conforter l'idée que, de manière plus globale, le vizir et la fonction qu'il exerce sont assimilés à des métaux autres que l'or. Dans l'Installation, le pharaon identifie directement le vizirat à du cuivre ayant pour fonction de créer une interface protectrice autour du souverain :

${ }{ }^{\mathbf{1}}$ Sa Majesté lui dit : Regarde vers cet office du vizir, ${ }^{2}$ sois vigilant concernant [tout] ce qui s'y fait. Vois, c'est le pilier du pays tout entier. Vois, pour ce qui concerne le vizirat, vois ce n'est pas quelque chose de doux. Vois, c'est une chose aussi amère que la bile. Vois, ${ }^{3}$ c'est le cuivre qui fait rempart autour de l'or du domaine [de] son [maitre] »

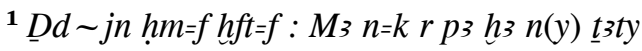
${ }^{2}$ rs-tp hr jrr[.t nb.t] jm=f. Mk smn pw n(y) t3 $r-\underline{d} r=f . M k j r \underline{t} 3 t y, m k n n$ bnr js pw. Mk dhr pw mj wdd. $M k^{3} h m t j p w \operatorname{mdr}(w) n b w n(y) \operatorname{pr}[n(y)$ $n b]=f(\text { TT 100, Installation, col. 1-3) })^{129}$

Ce passage, et plus généralement le programme textuel et iconographique du cycle viziral, témoigne du fait que le vizir est le protecteur et l'interlocuteur intermédiaire entre le souverain et le peuple. 
L'hypothèse émise par Helck sur la compréhension de ce passage du papyrus semble donc se confirmer, au moins du point de vue contextuel : l'or représente le roi et l'argent le vizir. Que ce soit sur les plans minéralogique, religieux et symbolique, architectural et manufacturier ainsi qu'économique et politique, le couple or/argent et roi/vizir permet d'assurer l'harmonie universelle et la cohésion des éléments, l'un complétant l'autre. L'assimilation plus globale du vizir à des métaux nous amène à nous interroger sur les raisons de ces assimilations du vizir à différents métaux ainsi que sur les critères sur lesquels repose le rapprochement avec tel métal ou tel autre.

\section{Datation}

Trois aspects de temporalité liée au papyrus Turin Cat. 1878 v $^{0}, 1.1-9$ sont à commenter : 1. la date de la nomination du vizir Ouseramon ; 2. la datation de la rédaction du texte et enfin 3. la datation de la composition originelle du texte.

\subsection{Date de la nomination du vizir Ous- eramon}

Notre papyrus mentionne "l'an $5,1^{\mathrm{er}}$ mois de la saison-akhet, le $1^{\text {er }}$ jour " de Thoutmosis III comme date de nomination d'Ouseramon en tant que vizir. En dépit du laps de temps séparant l'événement historique relaté ici et la période de sa rédaction sur le papyrus, la date exacte de la promotion d'Ouseramon devait être encore bien connue de la communauté de Deir al-Medineh de la période ramesside. Cependant, puisqu'il s'agit d'une oeuvre littéraire, il pourrait dès lors apparaître problématique de considérer cette date comme étant un fait historique certain. Elle pourrait également faire partie du processus de fiction littéraire déformant la réalité.

Néanmoins, une confirmation indirecte de la date de l'installation d'Ouseramon est donnée sur le verso d'une tablette en bois provenant probablement de Deir el-Bahari ${ }^{130}$. Il s'agit d'un registre comptable d'offrandes écrit en hiératique et daté du « $26^{\mathrm{e}}$ jour du $2^{\mathrm{e}}$ mois de la saison-péret de l'an 5 », vraisemblablement de Thoutmosis III. Une liste de contributeurs est mentionnée, dont le Pharaon, le domaine de la divine adoratrice, le héraut Antef, l'intendant Senenmout, le directeur du Trésor, le vizir Ouser et le domaine du vizir. Il s'agit probablement de la comp- tabilité préparatoire à une fête qui confirme donc qu'Ouseramon était déjà vizir au $26^{\mathrm{e}}$ jour du $2^{\mathrm{e}}$ mois de la saison-peret en l'an 5 de Thoutmosis III.

Ainsi, à l'aide de la tablette de Deir el-Bahari et sans argument contraire, Ouseramon était donc bien vizir, si ce n'est introduit comme tel, à compter de l'an 5 de Thoutmosis III, soit en 1472 av. J.-C. environ. Si nous prenons en compte les 30 ans de prêtrise qu'il semble avoir exercée auparavant, Ouseramon aurait débuté sa carrière au sein du temple d'Amon aux environs de 1502 av. J.-C., c'est-à-dire au mileu du règne de d'Amenhotep $\mathrm{I}^{\mathrm{er}}$.

Concernant le contexte politique, la transmission de la charge vizirale d'Âamtjou à son fils Ouseramon est directement liée à la situation inédite de l'exercice du pouvoir royal entre Thoutmosis III et Hatchepsout. En effet, de par ses nombreux membres et son réseau très étendu, la puissante famille d'Ouseramon a eu un poids politique et idéologique considérable dans la légitimation de la prise de pouvoir d'Hatchepsout, cette dernière alors régente devenant dès lors Pharaon, entre l'an 3 et l'an 7 de Thoutmosis III. Cette prise de pouvoir n'a en partie été possible qu'en s'appuyant, parmi d'autres, sur l'une des familles thébaines les plus influentes de l'époque, et cela principalement en maintenant leur haute position au sein des instances dirigeantes du pays. Ainsi, la promotion d'Ouseramon est pour Hatchepsout une façon de confirmer ses appuis à la cour et légitimer un peu plus sa prise de pouvoir ${ }^{131}$.

Cette famille continue de détenir le vizirat à la génération suivante lorsque Rekhmirê, le neveu d'Ouseramon, lui succède entre l'an 28 et l'an 34 de Thoutmosis III. Ouseramon a donc exercé ses fonctions vizirales durant 23 à 29 ans. Le floruit de cet homme est de ce fait considérable, le personnage étant au final l'un des acteurs majeurs des principaux événements politiques, économiques et religieux survenus au cours de cette période.

\subsection{Datation de la rédaction du texte et paléographie}

D'après l'espacement des signes, la fluidité des ligatures et le tracé des caractères individuels, notamment en ce qui concerne les signes D36 (1. 7, 9), D43 (1. 8), M4 (1. 1, 3, 4) et O11 (1. 9) de la Sign-List de Gardiner, Dorman, suivi par Dziobek, propose une 
paléographie ramesside (XIXe dynastie) ${ }^{132}$.

Cependant, aucun de ces signes ne présente réellement de caractéristiques justifiant une datation à la période suggérée. La datation de la rédaction de notre texte doit donc être requestionnée à la lumière des données issues de l'analyse matérielle et textuelle du document.

Tout d'abord, les dimensions du rouleau de papyrus étant celles couramment utilisées durant l'ensemble de la période ramesside, ces données ne constituent donc pas un critère discrimant permettant d'affiner la datation de la rédaction du texte (voir supra $\S 1.2$ ). Focalisons-nous ensuite sur les informations paléographiques. Bien que les principaux outils à notre disposition présentent certaines limites et doivent donc être maniés avec toute la prudence nécessaire ${ }^{133}$, il n'en demeure pas moins que le classement chronologique diachronique de la paléographie égyptienne réalisé par Möeller, complété depuis notamment par celui de Wimmer pour la période ramesside, reste l'instrument de référence afin de déterminer le cadre temporel plausible, plus ou moins fiable et précis, de la rédaction d'un texte.

Notre tableau paléographique du pap. Turin Cat. $1878 \mathrm{~V}^{0}$, 1. 1-9 (voir Annexe 1) ne semble pas confirmer l'hypothèse émise par Dorman et Dziobek. En effet, la forme des signes $g m(\mathrm{G} 28 ; 1.6), h$ (Aa1 ; 1.1 , 6 et 8), h3.t (F4; 1. 9), nbw (S12;1. 7 [1-2], 8 [1-2]) et $m$ (G17) dans la ligature $m^{-}$(1. 3), par exemple, sont caractéristiques de la $\mathrm{XX}^{\mathrm{e}}$ dynastie, et notamment de la première moitié de cette période.

Par ailleurs, les données issues de l'approche lexicale ${ }^{134}$ tendent à corroborer cette nouvelle proposition de datation mise en évidence au moyen du critère paléographique. Par exemple, à notre connaissance et en l'état actuel des sources à notre disposition, l'expression peu fréquente $\underline{d} d t$ jr.t (voir supra § 2, n. i) semble être attestée au plus tôt à la fin de la XIXe dynastie (règnes de Séthy II à Taousert) dans le pap. Anastasi I, 11, 4 (= pap. BM EA 10247). Elle se retrouve ensuite dans deux textes datés de la fin de la XXe dynastie (pap. Lansing, 3, 8 = pap. BM EA 9994 et pap. Bankes II, 4 = pap. BM EA 75017). Sa dernière mention apparaît à la fin de la XXVI dynastie dans le pap. Brooklyn 47.218.135, 5/17.

Par conséquent, nous concluons donc, d'une part, que la rédaction du papyrus n'est pas contemporaine de l'an 5 de Thoutmosis III, et d'autre part, qu'elle aurait été effectuée au cours de la première moitié de la $\mathrm{XX}^{\mathrm{e}}$ dynastie, c'est-à-dire entre les règnes de Sethnakht et Ramsès VII (1185-1123 av. J.-C. env.), soit sous l'un des vizirats suivants ${ }^{135}$ : Hori, Heouernef, To, Neferrenpet, Nehy ou Ramsesnakht, environ 260/320 ans après la mort d'Ouseramon.

\subsection{Datation de la composition originelle du texte}

Puisque nous concluons à une datation ramesside de rédaction du papyrus, se pose alors la question de la datation de la composition originelle du texte inscrit sur ce document.

Les mentions de l'an 5 de Thoutmosis III et du vizir Ouseramon, constituant uniquement un terminus post quem, et la paléographie ramesside ne permettent pas d'établir plus précisément la date de la composition originelle du texte. Il peut être la copie conforme ramesside d'un document administratif réalisé à l'occasion de l'accession d'Ouseramon au vizirat et datant donc de la XVIII ${ }^{e}$ dynastie - document pouvant, du reste, lui-même avoir été réalisé selon un modèle antérieur -, ou au contraire être une création originale ramesside ravivant le souvenir d'Ouseramon, et cela à partir d'une ou plusieurs sources d'information.

C'est selon la première hypothèse que Dorman a interprété le document qui, pour lui, est une copie ramesside exacte de ce qui était originellement un document administratif contemporain de la nomination d'Ouseramon ${ }^{136}$.

Pourtant, même si une date est mentionnée sur le papyrus, plusieurs éléments remettent en question l'hypothèse qu'il soutient :

- Le contenu (la circoncision, l'emploi métaphorique de l'or et de l'argent),

- Le registre linguistique (le discours direct, le registre de langue parlé, le jeu de question-réponse ${ }^{137}$,

- La langue (un aspect hybride mêlant néo et moyen-égyptien). Ce dernier point est d'ailleurs un élément caractéristique de certains registres littéraires du début de l'époque ramesside.

Ainsi, au vu de ces divers traits littérarisants, le texte ne peut pas être une copie ramesside exacte d'un document administratif contemporain de la nomination d'Ouseramon. 
Néanmoins, nous ne pouvons pas exclure qu'un acte administratif officiel ait pu servir de source d'information pour la rédaction de notre papyrus.

En complément, nous pouvons nous demander si les décors de la tombe d'Ouseramon (TT 131) n'ont pas aussi servi de source d'information pour la rédaction postérieure de notre texte. Or, comme nous l'avons précédemment souligné (voir supra § 3.1 et 3.3.1), la résonance entre le contenu de la version imagée et celui de la version littéraire ne laisse que peu de doute quant à l'utilisation de la sépulture du vizir.

Le papyrus est par conséquent une création littéraire originale ramesside ayant vraisemblablement été composée à partir de plusieurs sources d'informations contemporaines d'Ouseramon, et notamment à partir d'un acte administratif relatant effectivement sa nomination, mais aujourd'hui perdu, ainsi qu'à partir de la scène de la Nomination présente dans sa tombe (TT 131).

\section{Fonction du texte : magnifier la postérité du vizir Ouseramon}

Si nous postulons que notre texte n'est pas un document administratif, quelle est donc la véritable fonction d'une telle production littéraire, cela environ $260 / 320$ ans après la mort d'Ouseramon ${ }^{138}$ ?

De manière générale, il existe une tradition du souvenir pour les vizirs ayant précédé l'époque ramesside. Celle-ci apparaît au sein de différentes sphères : postérité dans le cercle familial, hommage rendu par un subalterne dans le cadre professionnel et mémoire des grandes figures de l'Histoire.

Se pose alors la question de savoir quel vizir est une figure historique et quel autre ne l'est pas. En l'état actuel de la documentation, il reste difficile d'établir précisément l'ensemble des critères justifiant la persistance du souvenir historique pour certains, et non pour d'autres. Citons deux exemples d'évocation du souvenir de figures historiques.

Au tout début de la XIX dynastie (règnes de Ramsès $\mathrm{I}^{\mathrm{er}}$ et Séthy I $\left.{ }^{\mathrm{er}}\right)$, le grand prêtre du $k a$ royal de Thoutmosis I ${ }^{\mathrm{er}}$, Ouserhat, affirme être le descendant du vizir Imhotep (début XVIII ${ }^{e}$ dynastie, règne de Thoutmosis ${ }^{\mathrm{er}}$ ), dans la généalogie « fictive » représentée dans sa tombe, située dans la nécropole thébaine de Cheikh abd el-Gournah (TT 51) ${ }^{139}$.

Le vizir Imhotep apparait aussi sur le fragment
Daressy XIX $^{\mathrm{e}}$ dynastie, daté vraisemblablement entre l'an 26 et l'an 55 du règne de Ramsès $\mathrm{II})^{140}$ provenant d'une tombe ramesside située dans la nécropole de Saqqâra. Le document expose une sélection d' " hommes illustres », figurés et/ou mentionnés, répartis en cinq séries hiérarchiquement ordonnées, dont une série de huit vizirs (seulement cinq sont conservés), parmi lesquels figurent les vizirs Imhotep et Ousermonthou pour la XVIII ${ }^{e}$ dynastie. Considérant l'importance d'Ouseramon, nous pouvons nous étonner du fait qu'il ne soit pas représenté sur le fragment Daressy, et de ce fait, nous interroger sur la postérité de ce dernier.

Toutefois, à Cheikh Abd el-Gournah, sa sépulture est une tombe double monumentale (TT 131 et TT 61), dont les deux entités sont respectivement situées dans la partie inférieure et supérieure la colline. En outre, la façade de la TT 131 est à redans et la tombe était surmontée d'une pyramide, ce qui sans aucun doute signe l'importance du personnage et a contribué à faire perdurer le souvenir d'Ouseramon bien au-delà du seul règne de Thoutmosis III $^{141}$.

De surcroît, l'élaboration littéraire dont a fait l'objet Ouseramon à l'époque ramesside dans notre papyrus est directement associée au souvenir du souverain sous lequel il a servi, c'est-à-dire Thoutmosis III. Les exploits militaires du roi lors de ses nombreuses campagnes menées en Syro-Palestine sont notamment relatés dans les Annales gravées sur les murs du grand temple de Karnak. La première expédition, effectuée en l'an 23 à destination de Megiddo, y est particulièrement développée et une Königsnovelle est même insérée au sein de la section correspondante à cet événement ${ }^{142}$.

Ce remarquable passé a particulièrement retenu l'attention de Ramsès II, puisque ce dernier a largement repris les éléments de la phraséologie créée pour Thoutmosis III dans ses propres monuments. De même, c'est Thoutmosis III qui est choisi lorsqu'il s'agit d'évoquer un souverain de référence dans les élaborations littéraires de l'époque ramesside, comme l'attestent le récit de La Prise de Joppé (papyrus Harris 500 = pap. BM EA 10060, rédigé au début de la XIXe dynastie) ainsi que celui de Thoutmosis III en Asie (pap. Turin Cat. 1940-1941, rédaction estimée au cours des XIX'-XX' dynasties) ${ }^{143}$. Par ailleurs, le prince Khâemouaset ${ }^{144}$ comme le vizir Paser - 
qui reprend, par exemple, les Devoirs du vizir dans sa tombe (TT 106) $)^{145}$-, ont eux aussi manifesté un intérêt historique marqué pour le milieu de la XVIII dynastie.

Or, quand au cours de la première moitié de la $\mathrm{XX}^{\mathrm{e}}$ dynastie, il est question de se remémorer un vizir au sein d'une production littéraire, le choix de recourir à celui de la première partie du règne de Thoutmosis III - et pas un autre - témoigne de la persistance du souvenir du vizir Ouseramon jusqu’à l'époque ramesside. De même que Thoutmosis III est perçu comme un souverain de référence, Ouseramon est le vizir retenu afin d'être relié à cet inoubliable passé. De plus, le choix d'associer cette élaboration littéraire à l'Hymne à Hâpi n'est peut-être pas uniquement dû au hasard, eu égard au fait que l'hymne semble avoir été créé au début de la XVIII dynastie ${ }^{146}$.

La Nomination du vizir Ouseramon consignée sur ce papyrus relève donc des textes qui ont transcendé les faits historiques. Ceux-ci deviennent alors des éléments constitutifs d'oeuvres fictionnelles puisant dans un répertoire de références historiques et culturelles communes connues et sans cesse réadaptées $^{147}$.

La mise en scène de la nomination du vizir Ouseramon relatée au verso du pap. Turin Cat. 1878 est donc une élaboration littéraire ramesside, au contenu original (circoncision, métaphore or/argent) et sans exemple analogue direct, s’inscrivant, de manière plus générale, dans le mouvement ramesside du « rapport au passé » selon l'expression employée par Assmann ${ }^{148}$. Cette création, associée au glorieux règne de Thoutmosis III et centrée sur son vizir, s'inspire essentiellement de sources d'information contemporaines (acte administratif officiel et TT 131), et constitue un témoin majeur de l'importance d'Ouseramon dans l'histoire de son pays et du vizirat.

\section{Notes}

${ }^{1}$ Maruéjol, Thoutmosis III, 2014², p. 168-79 ; B.M. Bryan, dans Cline et O'Connor (éd.), Thutmose III, 2006, p. 69-77 ; Dziobek, Denkmäler Vezirs User-Amun, 1998, (notamment p. 85-101 pour la liste des monuments appartenant au vizir ou le mentionnant) ; Dziobek, Gräber Vezirs User-Amun, 1994 ; Hornung, Grabkammer Vezirs User, 1961 ; Helck, Verwaltung, 1958, p. 290-93, 436-37 [5].

${ }^{2}$ Il le mentionne dans sa seconde lettre adressée au duc de Blacas lorsqu'il visite le musée de Turin en 1824, voir Champollion, Lettres à M. le duc de Blacas d'Aulps, II, 1826, p. 57-58 et pl. IX [8].

${ }^{3}$ Ce travail a bénéficié du soutien du LabEx ARCHIMEDE au titre du programme « Investissement d'Avenir » ANR-11-LABX-0032-01. Je tiens, en premier lieu, à remercier chaleureusement $\mathrm{A}$. Stauder pour nos enrichissantes discussions ainsi que pour ses suggestions des plus précieuses, particulièrement en ce qui concerne les commentaires de traduction. Je remercie également vivement M. Gabolde pour l'encadrement de ma recherche doctorale au sein de laquelle s'insère directement cet article. F. Contardi a toujours été prompt à répondre à toutes mes questions. Enfin, que soient chaudement remerciées mes collègues et avant tout amies Virginie, Magali et Charlène pour leurs conseils et corrections. Les erreurs et oublis sont la seule responsabilité de l'auteur.

${ }^{4}$ Pleyte et Rossi, Les papyrus hiératiques de Turin, I-II, 1869-1876, p. 4-6 et pl. I.

${ }^{5}$ Fabretti et al., Catalogo Generale dei Musei di Antichità, I, 1882, p. 240 [Cat. 1878].

${ }^{6}$ Bellion, Catalogue des manuscrits hiéroglyphiques et hiératiques, 1987, p. 266-67, 283.
${ }^{7}$ DZA 24.458.560 et 24.458.570 (mts ; TLA Lemma $\mathrm{n}^{\circ} 77840$ ) et DZA 31.444 .790 ( $d h n$ ? ; TLA Lemma $\left.\mathrm{n}^{\text {os }} 854587,180280,180290\right)$, accessibles sur le site internet du Thesaurus Linguae Aegyptiae (TLA) : http://aaew.bbaw.de/tla/ (consulté le 12/03/2018).

${ }^{8}$ Helck, Urk. IV, $1984{ }^{2}$, 1384, 3-20 ; Helck, dans Firchow (éd.), Ägyptologische Studien, 1955, p. 112, fig. 3.

9 Černý, Notebook MSS 17.151, p. 35 (rédigé durant l'entre-deux-guerres ou juste après la Seconde Guerre mondiale). Voir http://archive.griffith.ox.ac.uk/index. php/notebooks-2 et http://archive.griffith.ox.ac.uk/ index.php/notebook-cerny-mss-17-151 (consultées le 29/01/2018).

${ }^{10}$ Helck, Urk. IV. Übersetzung, 1961, p. 71 = Cumming, Egyptian Historical Records, II, 1984, p. 97 ; Helck, Verwaltung, 1958, p. 291 (trad. 1. 1-5), 436 [5.7] ; Helck, dans Firchow (éd.), Ägyptologische Studien, 1955, p. 111-12, 114-15 (notes de traduction).

${ }^{11}$ Dziobek, Denkmäler Vezirs User-Amun, 1998, p. 98-99.

${ }^{12}$ Dorman, dans Cline et O'Connor (éd.), Thutmose III, 2006, p. 46 ; Dziobek, Denkmäler Vezirs User-Amun, 1998, p. 98 ; Dorman, The Monuments of Senenmut, 1988, p. 33-34.

${ }^{13}$ Auenmüller, Die Territorialität der Ägyptischen Elite(n), 2013, p. 819-20 [WS18-5, s] ; Hagen, Ancient Egyptian Literary Text in Context, 2012, p. 195 ; Černý, Community of Workmen, $2001^{2}$, p. 308, n. 2 ; Laboury, La statuaire de Thoutmosis III, 1998, p. 18 ; Anonyme, dans Helck et Westendorf (éd.), LÄ IV, 1982, col. 734 [12.], s.v. « Papyri Turin »; Vernus, RdE 33 (1981), p. 89-124 ; Möller, ZÄS 56 (1920), p. 37 [H. 1.] ; Petrie, A History of Egypt, II, 1899³, p. 100 ; Erman, Die Märchen des Papyrus Westcar, II, 1890, p. 35 [6.] ; Wiedemann, Ägyptische Geschichte, I, 1884, p. 346. 
${ }^{14} \mathrm{Je}$ tiens à remercier S. Töpfer, conservatrice et responsable de la collection des papyrus du Museo Egizio pour m'avoir communiqué les photographies nécessaires à la réalisation de cet article. Les photographies seront également librement accessibles sur internet via le projet « The Turin Papyrus Online Platform » (TPOP). Lire à ce sujet Töpfer, Rivista del Museo Egizio 2 (2018), p. 1-11 (accessible sur le site internet de la revue à l'adresse : https://doi. org/10.29353/rime.2018.1916, dernière consultation le 24/12/2018); Del Vesco et Poole, dans Dorn et Polis (éd.), Outside the Box, 2018, p. 97-130.

${ }^{15}$ Notons que nous avons délibérément omis les traces de signes n'appartenant pas au texte de la Nomination du vizir Ouseramon afin de présenter un fac-similé le plus lisible possible dans le cadre de cet article centré sur ce texte en particulier.

${ }^{16}$ Anonyme, dans Helck et Westendorf (éd.), LÄ IV, 1982, col. 734 [12.], s.v. « Papyri Turin »; Bacchi, L'Inno al Nilo, [s.d.], p. 1 ; Fabretti et al., Catalogo Generale dei Musei di Antichità, I, 1882, p. 264 [2027].

${ }^{17}$ Fragment $n^{\circ} \mathrm{c}$ (= pap. Tur. Cat. 1878 vo IV,1-2) $: 5 \mathrm{~cm}$ de hauteur et $2,4 \mathrm{~cm}$ de largeur. Fragment no b (= pap. Tur. Cat. 1878 vo IV,6-9) $: 8,2 \mathrm{~cm}$ de hauteur et $5,4 \mathrm{~cm}$ de largeur.

${ }^{18}$ Roccati, dans Donadoni Roveri (éd.), Passato e futuro del Museo Egizio di Torino, 1989, p. 120-28 ; Roccati, BSFE 99 (1984), p. 9-27. Sur l'histoire du musée et de ses collections, voir aussi Curto, Storia del Museo Egizio di Torino, 1976 (notamment p. 41-48). Pour la provenance, voir également Demichelis, VicOr 11 (1999), p. 103 et n. 1 ; Valbelle, "Les ouvriers de la tombe", 1985, p. 28.

${ }^{19}$ Voir notamment Stauder, Linguistic Dating of Middle Egyptian Literary Texts, 2013, p. 215-36 (§ 3.4) ; Van der Plas, dans Moers et al. (éd.), Dating Egyptian Literary Texts, 2013, p. 471-82 (avec la bibliographie mise à jour depuis la publication de référence datant de 1986) ; Van der Plas, L'hymne à la crue du Nil, I-II, 1986 (avec toute la bibliographie antérieure, vol. I, p. 3-9); Assmann, dans Helck et Westendorf (ed.), LÄ IV, 1982, col. 489-96, s.v. « Nilhymnus »; Barucq et Daumas, Hymnes et prières de l'Égypte ancienne, 1980, p. 493-501 [156] ; Lichtheim, Ancient Egyptian Literature, I, 1973, p. 204-10 ; Helck, Der Text des "Nilhymnus", 1972 (et compte-rendu dans Meeks, BiOr 32/1 [1975], p. 18-25) ; Erman, ZÄS 85 (1960), p. 35-42 ; Wilson, dans Pritchard (éd.), $A N E T^{2}, 1955^{2}$, p. 372-73. Pour la version des papyrus de Turin en particulier, voir Anonyme, dans Helck et Westendorf (éd.), LÄ IV, 1982, col. 733 [4.-5.], 734 [12.], s.v. "Papyri Turin »; Roccati, OrAnt 14/3 (1975), p. 245 [7] (= pap. CGT 54016) ; Bacchi, L'Inno al Nilo, [s.d.] ; Grapow, ZÄS 52 (1914), p. 103-6 (= pap.Tur.Cat. 1968 $\mathrm{r}^{\circ}$ et $1890 \mathrm{r}^{\circ}$, en se référant, à tort, à Pleyte et Rossi, Les papyrus hiératiques de Turin, II, 1869-1876, pl. CLIV b pour la transcription du $1^{\mathrm{er}}$ texte) ; Maspero, Hymne au Nil, 1912, p. XII, 20 [III] (= pap.Tur.Cat. 1968 ro d'après la transcription de Gardiner communiquée, à la demande de celui-ci, par Grapow) ; Fabretti et al., Catalogo Generale dei Musei di Antichità, I, 1882, p. 243 [1890] (= pap.Tur.Cat. 1890 ro), 257 [1968] (= pap.Tur.

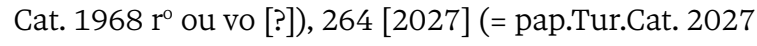
$\mathrm{r}^{\circ}$ ou vo [?]) ; Pleyte et Rossi, Les papyrus hiératiques de Turin, I-II, 1869-1876, p. 82 et pl. LXIV (= pap.Tur.Cat. $1890 \mathrm{r}^{\circ}$ ). Consulter aussi l'édition de P. Dils, accessible en ligne sur le TLA : http://aaew.bbaw.de/tla/servlet/ OTPassport? $\mathrm{u}=$ guest $\& \mathrm{f}=0 \& \mathrm{l}=0 \& \mathrm{OC}=544 \& \mathrm{db}=0$ et http://aaew.bbaw.de/tla/servlet/GetCtxt?u=guest\&f= $0 \& \mathrm{l}=0 \& \mathrm{tc}=545 \& \mathrm{db}=0 \& \mathrm{ws}=0 \& \mathrm{mv}=1$ (dernière consultation le 12/12/2018).

${ }^{20}$ Anonyme, dans Helck et Westendorf (éd.), LÄ IV, 1982, col. 734 [12.], s.v. " Papyri Turin »; et cité par Dorman, The Monuments of Senenmut, 1988, p. 33-34.

${ }^{21}$ Informations communiquées par Federico Poole, Susanne Töpfer et Juan José Archidona Ramirez suite à l'examen de l'original.

${ }^{22}$ Identification datant du 14 septembre 1983 . Voir Roccati, Archaeogate (2008), p. 1 [i.] (article publié en ligne le 16/06/2008 - http://www.archaeogate. org/egittologia/article.php?id=283 [URL non valide à présent]. Je remercie vivement $\mathrm{F}$. Contardi pour la communication de cette référence bibliographique); Roccati, dans Pennacchietti et Roccati (éd.), Atti della terza giornata di studi camito-semitici e indoeuropei, 1984, p. 113-14 note qu'il avait récemment « identifié un autre fragment appartenant à une copie différente de la même oeuvre (i.e. Satire des Métiers) et qui rapporte un passage proche de celui étudié ici (i.e. CGT 54017) ». Il fait vraisemblablement allusion au texte du papyrus CGT 54018.

${ }^{23}$ Vernus, Sagesses de l'Égypte pharaonique, 2010², p. 239-64 (notamment la bibliographie antérieure p. 242, n. 3-6, 255, n. 1) ; Jäger, Altägyptische Beruftypologien, 2004 ; Mathieu, Grafma Newsletter 3-4 (2001), p. 65-73 ; Mathieu, Grafma Newsletter 2 (1998), p. 37-40.

${ }^{24}$ Pleyte et Rossi, Les papyrus hiératiques de Turin, I-II, 1869-1876, p. 215 et pl. CLIV b (= pap.Tur.Cat. $\left.1968 \mathrm{v}^{0}\right)$.

${ }^{25}$ DZA 23.795.950 (concernant les entrées TLA Lemma nos 67570/67590, s.v. «m3t $/ m 3 w \underline{t} »)$, accessible à l'adresse : http://aaew.bbaw.de/tla/ (consulté le 06/08/2018).

${ }^{26}$ Pleyte et Rossi, Les papyrus hiératiques de Turin, II, 1869-1876, pl. I.

${ }^{27}$ Concernant les problématiques liées à la matérialité, consulter notamment les récentes publications de Hoogendijk et van Gompel (éd.), The Materiality of Texts from Ancient Egypt, 2018 ; Krutzsch, dans Feder et al. (éd.), Ägypten begreifen, 2017, p. 213-22 ; Krutzsch, BMSAES 23 (2016), p. 57-69; Krutzsch, dans Fischer-Elfert, Magika Hieratika, 2014, p. 1-74 ; Piquette et Whitehouse (éd.), Writing as Material Practice, 2013 ; Bülow-Jacobsen, dans Bagnall (éd.), The Oxford Handbook of Papyrology, 2009, p. 3-29 (part. p. 4-10, 18-23); Krutzsch, dans Backes et al. (éd.), Totenbuch-Forschungen, 2006, p. 167-95.

${ }^{28}$ Ménei, dans Laroque (dir.), Autour des papiers asiatiques, 2017, p. 138-60 (mis en ligne à l'adresse https://hicsa.univ-paris1.fr/documents/pdf/ PublicationsLigne/Actes\%20Laroque\%202017/08_ Menei.pdf, dernière consultation le 17/12/2018); Parkinson et Quirke, Papyrus, 1995, p. 13-14 ; 
Grandet, Le papyrus Harris I (BM 9999), I, 1994, p. 29 ; Ménei, $R d E 44$ (1993), p. 185-88 ; Turner, dans Bingen et Nachtergael (éd.), Actes du XV Congrès International de Papyrologie I, 1978 ; Černý, Papers and Books, 1947, p. 10, 17. Lire également Leach et Tait, dans Nicholson et Shaw (éd.), Ancient Egyptian Materials and Technology, 2000, p. 227-53.

${ }^{29}$ Parkinson et Quirke, Papyrus, 1995, p. 16-17 ; Černý, Papers and Books, 1947, p. 16-17.

${ }^{30}$ Grandet, Le papyrus Harris I (BM 9999), I, 1994, p. 29 ; Černý, Papers and Books, 1947, p. 9.

${ }^{31}$ Sur cette problématique lire, entre autres, Friedrich et Schwarke (éd.), One-Volume Libraries. Composite and Multiple-Text Manuscripts, 2016.

${ }^{32}$ Sur la culture scribale écrite et orale en Égypte antique, et plus particulièrement ici à propos de la communauté de lettrés du village de Deir al-Medineh ayant vécu au cours de la première moitié de la $\mathrm{XX}^{\mathrm{e}}$ dynastie, consulter, entre autres, Dorn et al., dans Dorn et Polis (éd.), Outside the Box, 2018, p. 7-16; Vernus, dans Dorn et Polis (éd.), Outside the Box, 2018, p. 477-92 ; Winand, dans Dorn et Polis (éd.), Outside the Box, 2018, p. 493-524 ; Grossman et Cromwell, dans Cromwell et Grossman (éd.), Scribal Repertoires in Egypt, 2018, p. 1-19; Ryholt, dans Cromwell et Grossman (éd.), Scribal Repertoires in Egypt, 2018, p. 153-83; Hagen, dans Bausi et al. (éd.), Manuscripts and Archives, 2018, p. 71-170 ; Maltomini, dans Derda et al. (éd.), Proceedings of the $27^{\text {th }}$ International Congress of Papyrology, 2016, p. 1097-112 ; Pinarello, Archaeological Discussion of Writing Practice, 2015 ; Hagen, JARCE 49 (2013), p. 82-91; Enmarch et Lepper (éd.), Ancient Egyptian Literature, 2013 ; Goelet Jr., dans Cline et O’Connor (éd.), Ramesses III, 2012, p. 305-403 ; Schmidt, dans Somers et Coulie (éd.), Palimpsestes et édition de textes, 2009, p. 83-99; Goelet Jr., dans D’Auria (éd.), Servant of Mut, 2008, p. 102-10 ; Schmidt, dans Frösén et al. (éd.), Proceedings of the $24^{\text {th }}$ International Congress of Papyrology, II, 2007, p. 979-90 ; Allon et Navratilova, Ancient Egyptian Scribes, 2007 ; Baines, dans Baines, Visual and Written Culture in Ancient Egypt, 2007, p. 146-78 ; Hagen, dans Mairs et Stevenson (éd.), Current Research in Egyptology 2005, 2007, p. 38-51 ; Hagen, dans Dann (éd.), Current Research in Egyptology 2004, 2006, p. 8-99 ; Haring, dans Dorn et Hofmann (éd.), Living and Writing in Deir el-Medine, 2006, p. 107-12 ; Haring, JESHO 46 (2003), p. 249-72 ; Donker van Heel et Haring, Scribal Practice in Ramesside Deir el-Medina, 2003 ; Quirke, dans Loprieno (éd.), Ancient Egyptian Literature, 1996, p. 379-401 ; Janssen, dans Demarée et Egberts (éd.), Village Voices, 1992, p. 81-94; Goody, The Interface Between the Written and the Oral, 1987 ; Caminos, dans Bierbrier (éd.), Papyrus. Structure and Usage, 1986, p. 43-61.

${ }^{33}$ Gauthier, Le livre des Rois, II, 1912, p. 254 [III] et n. 2.

${ }^{34}$ Helck, Urk. IV, $1984{ }^{2}$, 1384, 4 ; Helck, dans Firchow (éd.), Ägyptologische Studien, 1955, p. 111-12 fig. 3. Dziobek, Denkmäler Vezirs User-Amun, 1998, p. 98 suit la transcription de Helck et traduit $s \underline{t} z$ par « Hereinführen » (« introduction »). Pour st 3 voir Wb IV, 353, 18-354, 4 ; AnLex 77.3994, 78.3959 ;
Wortdiskussionen, https://aegyptologie.philhist.unibas. ch/fileadmin/user_upload/aegyptologie/Forschung/ Werkzeuge/Wortdiskussionen/Wortdiskussionen_ Gesamtdokument.pdf, s.v. «stż »; FCD, 1962, p. 255 ; Möller II, 519.

${ }^{35}$ Tombe d'Ouseramon (TT 131), Nomination du vizir, 1.2 = Helck, Urk. IV, 1984², 1380, 14 ; Dziobek, Denkmäler Vezirs User-Amun, 1998, p. 3 et pl. 1.

${ }^{36}$ Pleyte et Rossi, Les papyrus hiératiques de Turin, I, 1869-1876, p. 4 ; Černý, Notebook MSS 17.151, p. 35. Pour $b s$ voir VÉgA ID 2246 (pour « Vocabulaire de l'Égyptien Ancien », consultable à l'adresse http://vega-vocabulaire-egyptien-ancien.fr/ acceder-a-loutil/) ; Wb I, 473, 1-18 ; AnLex 77.1308, 78.1358, 79.0928; Wortdiskussionen, https://aegyptologie.philhist.unibas.ch/ fileadmin/user_upload/aegyptologie/Forschung/ Werkzeuge/Wortdiskussionen/Wortdiskussionen_ Gesamtdokument.pdf, s.v. « bs »; FCD, 1962, p. 84 ; TLA Lemma $n^{\circ} 57160$ (dernier accès le 02/02/2018); Möller II, 255.

${ }^{37} \mathrm{TT}$ 131, Nomination du vizir, col. 24 et légende d'Ouseramon (= Dziobek, Denkmäler Vezirs UserAmun, 1998, p. 7 et pl. 1 ; Dziobek, Gräber Vezirs User-Amun, 1994, p. 75 et pl. 72 [texte 5h]) et cône funéraire Macadam 370 (= Dziobek, Denkmäler Vezirs User-Amun, p. 88, 101 ; Davies et Macadam, Funerary Cones, I, 1957, p. 21 [370]). Pour ce titre, voir Al. Ayedi, Index, 2006, p. 570 [1911-1913] ; Quirke, Titles and Bureaux, 2004, p. 78, 122, 127 ; Eichler, Verwaltung des " Hauses des Amun », 2000, p. 135-37, 265 [175] ; Jones, Titles, II, 2000, p. 865 [3167] ; Ward, Index, $1982, n^{\text {os }} 1434-36$. Voir Möller II, 422 pour le signe htm. Son fils Merymaât porte également le titre de š̌ htm-ntr (ny Jmn) sur le cône funéraire Macadam 1 (= Davies et Macadam, Funerary Cones, I, 1957, p. 21 [1]) et dans les graffiti E.2.10 et O.2.7 inscrits dans la grotte des scribes (tombe MMA 504) à Deir el-Bahari (= Ragazzoli, La grotte des scribes à Deir el-Bahari, 2017, p. 63, 154, fig. 4.11, 155-156 [E.2.10], 186-188 [O.2.7] et fig.). Son autre fils Samenkhet porte lui aussi le titre de $s \breve{s}$ htm-ntr $n(y)$ Jmn, cette fois, dans la tombe de son cousin le vizir Rekhmirê (= Davies, Tomb of Rekh-mi-Rēe, 1943, pl. IX [bas]). Enfin, Merymaât ou Samenkhet porte le titre de š̌ htm-ntr $[n(y) J m n]$ dans la TT 131 (= Dziobek, Denkmäler Vezirs User-Amun, 1998, p. 122 et pl. 96 [scène 131-16b]).

${ }^{38}$ Helck, Urk. IV, 1984² , 1384, 6 ; Helck, dans Firchow (éd.), Ägyptologische Studien, 1955, p. 112 fig. 3, 115. Voir également les expressions $j b=j r$ jr.t $s p[. .$.$] et r h n$ $j b=j h r[. .$.$] prononcées par le roi dans la scène de la$ Nomination de Qenamon (TT 93, règne d'Amenhotep II) : Davies, The Tomb of Ken-Amūn at Thebes, I, 1930 pl. VIII, col. 5-6.

${ }^{39}$ Dziobek, Denkmäler Vezirs User-Amun, 1998, p. 98-99.

${ }^{40}$ Helck, Urk. IV, 1984² , 1384, 7 ; Helck, dans Firchow (éd.), Ägyptologische Studien, 1955, p. 112 fig. 3 ; Černý, Notebook, MSS 17.151, p. 35 ; Gardiner, Egyptian Grammar, $1957^{3}, \S 502$.

${ }^{41}$ « [...] zustimmen <zu> User-Amun, Sohn des Amos. Wie viele Jahre [...]? » : Quack, dans Berlejung et al. (éd.), Menschenbilder und Körperkonzepte, 2012, p. 595-96 
d'après l'étude étymologique de Schneider, Asiatische Personennamen, 1992, p. 71-72 [N 129] pour le nom Âamtjou. Quack reconnait et concède toutefois que le signe problématique en question est endommagé sur sa partie droite par un petit trou.

${ }^{42} \mathrm{Grammaticalement}$, cette proposition serait plutôt à analyser comme suit : $\varnothing \boldsymbol{\zeta}_{3}(=w): m \underline{t} 3(=f)$, littéralement « c'est grand : (son) phallus ». Voir par ailleurs la fiche manuscrite du Wörterbuch TLA DZA 24.458.570, accessible à l'adresse http://aaew.bbaw.de/tla/ (consultée le 10/12/2018), concernant l'entrée $m \underline{t} 3$ «phallus » (TLA lemma ${ }^{\circ} 77840$ ) répertoriée pour le papyrus Turin Cat. 1878 v , 1. 1-9 (TLA DZA 24.458.560). Le rédacteur de la fiche se demandait déjà si l'expression écrite sous la forme '3-mt3, signifiant pour lui « gros phallus », ne ferait pas également penser au nom personnel Âamtjou ( $3 m \underline{t} w$ ).

${ }^{43}$ Se référer également à Wimmer, Hieratische Paläographie, 1995, p. 32 (signe $r$ ), 356 (signe $t$ sur celui de l'oeuf). Notons toutefois que la partie gauche du premier oeuf du pap. Abbott recensé par Möller ressemble également à celui présent dans notre manuscrit. La rédaction du texte du papyrus Abbott est en revanche postérieure à celui étudié dans le cadre de cet article puisqu'elle date du règne de Ramsès IX, soit à la toute fin de la XX dynastie. ${ }^{44}$ Černý, Notebook, MSS 17.151, p. 35.

${ }^{45}$ VÉgA ID 1224, s.v. « 3.t» ; Wb I, 163, 12-14, s.v. « 3.t»; AnLex 77.0561, 78.0614, 79.0415, s.v. « अ3.t»; FCD, 1962, p. 37, s.v. « 3.t»; TLA Lemma n 34860, s.v. « $3 . t »$ (dernier accès le 04/03/2019).

${ }^{46}$ VÉgA ID 3480, s.v. «r/jr»; ID 1224, s.v. « $3 . t »$ et ID

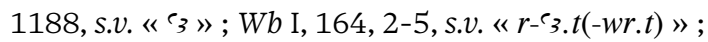
AnLex 77.0563 (r-?.t-wr.t), $79.0416\left(r-c_{3}-w r(. t)\right) ; F C D$, 1962, p. 37, s.v. « 3.t»; Lesko, DLE', II, 2004, p. 48, s.v. «r-3.t-wr.t»; Hannig, $\ddot{A} W b, \mathrm{I}, 2003$, p. $257\{4725\}$, s.v. «r-`3.t(-wr.t)»; Hannig, $\ddot{A} W b, \mathrm{II} / 1,2006$, p. 479

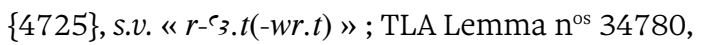
s.v. « '3.w » et 34860, s.v. « 3.t» (dernier accès le 04/03/2019) ; Gardiner, Egyptian Grammar, 1957³, p. 156 [§ 205,5] (r-§.t); Grundriss VII/1, 1961, p. 122, s.v. «r-? 3.t» et " $r$-`3.t-wr.t». Voir par exemple les légendes de la scène $\mathrm{X}$ du mythe de la naissance divine à Deir el-Bahari dans laquelle l'enfant (la future Hatchepsout roi) est présenté à son père divin Amon par la déesse Hathor. Légende du dieu Amon : « Ce noble dieu vient pour voir sa fille qu'il aime, le roi de Haute et Basse-Égypte (Maâtkarê)|, vivante, après qu'elle est née. Et alors son coeur se réjouit grandement » ([jj $n$ ntr pn spš $]$ r m33 s3.t=s $\operatorname{mrr}(w) . t=f$

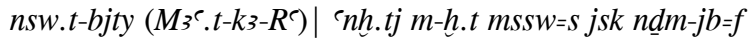
$r$-3.t-wr.t). Légende de la déesse Hathor : «Qu'elle place/présente son bras devant Sa Majesté (afin de) présenter/dévoiler/révéler(?) l'oisillonne (i.e. l'enfant féminin héritière) grandement " $\left(d=s \varsigma^{c} s h f t h m=f s n z(?)\right.$ $\underline{t}$ 3.t $r$ - $3 . t$-wr.t $)=$ Coyette, dans Cannuyer et Vialle (éd.), Les naissances merveilleuses en Orient, 2015, p. 100 ; Sethe, Urk. IV, 1961 3 , 228, 4 et 7 (r-3.t-wr.t) = Sethe, Urk. IV. Übersetzung, 1914, p. 106 ; Naville, Deir el Bahari II, 1895, pl. LII. Voir également comme deuxième exemple, dans la tombe de Iamounedjeh (premier héraut royal et directeur de la porte, TT
84, règne de Thoutmosis III), la légende du tableau mettant en scène des Asiatiques apportant leurs tributs au roi : "Apparition du roi sur le grand trône dans le palais d'Héliopolis du Sud. Son coeur se réjouit grandement en bravoure et en victoire. Après cela, apporter les tributs (...) » (he.t nsw.t $m$ s.t wr.t $m$ `h nyw Jwnw $\breve{S} m$ r. $3 w-j b=f r-e_{3} . t-w r . t m$ qn.t $m$ nh.t. M-h.t nn jn.t jn.w (...)) = Sethe, Urk. IV, 1961 3 , 951, 4 = Blumenthal et al., Urk. IV, Übersetzung, 1984, p. 349-51 ; PM I/1², 1960, p. 168-69 (9).

${ }^{47} \mathrm{~Wb}$ I, 164, 2-3 = Wb Beleg I, p. 30 [3]. Notons qu'ils considèrent que notre papyrus date de la XVIII dynastie.

${ }^{48}$ Dziobek, Gräber Vezirs User-Amun, 1994, p. 73-75 et pl. 81 [scène 131-5], p. 75-77 et pl. 82 [scène 131-6], p. 98 et pl. 97 [131-17] et p. $98-99$ et pl. 98.

${ }^{49}$ Polz, Der Beginn des Neuen Reiches, 2007, p. 282-84 ; Dziobek, Denkmäler Vezirs User-Amun, 1998, p. 103-06 et pl. 10 ; Kampp, Thebanische Nekropole, I, 1996, p. 330-33 et fig. 213-214 ; PM I/12 , 1960, p. 167 et plan p. 160 ; Helck, Verwaltung, 1958, p. 435 [4, a]. Les principales dégradations modernes proviennent notamment du fait que, durant le XIX siècle, la tombe a longtemps servi de maison de fouilles ainsi que de résidences à plusieurs égyptologues tels que Wilkinson, Hay, Hogg et son ami Fresnel, puis Lepsius suivi par Rhind et ensuite par l'artiste américain Smith. La tombe reste d'ailleurs de nos jours connue comme étant « la maison de Wilkinson ».

${ }^{50}$ Dans les autres sources concernant Ouseramon, lorsque sa filiation paternelle sous la forme Âamtjou est précisée, Ouseramon est soit $j r \sim n j m y-r 3$ njw.t $\underline{t}$ żty soit sz jmy-r3 njw.t țzty : Dziobek, Denkmäler Vezirs User-Amun, 1998, pl. 11a-c, e, g ; Dziobek, Gräber Vezirs User-Amun, 1994, pl. 98 ; Caminos et James, Gebel es-Silsilah, I, 1963, pl. 47 ; Davies et Macadam, Funerary Cones, I, 1957, p. 21 [358, 370].

${ }^{51} \mathrm{~Wb}$ II, 175,5 , s.v. « $m \underline{t} 3$ »; TLA Lemma no 77840, s.v. « $m \underline{t} 3$ » et DZA 24.458.560, 24.458.570, accessible à l'adresse http://aaew.bbaw.de/tla/ (consultée le 15/03/2019).

${ }^{52}$ Demarée, Ramesside Ostraca, 2002, p. 20 [5672] et pl. 44 ; KRI VI, 1983, 660, 13 [A.27] = KRITA VI, 2012, p. 468 [A.27] ; Ward, dans Leonard Jr. et Williams (éd.), Essays in Ancient Civilization, 1989, p. 296 [5.]. Remarquons cependant que la graphie ne présente pas le signe $t$, détail qui a son importance dans la discussion problématique de cet article.

${ }^{53}$ Schneider, Asiatische Personennamen, 1992, p. 290 [F 8]. ${ }^{54}$ O. CGC 25209 ro 1.2 (provient de la tombe de Ramsès VI, usurpé de Ramsès V, KV 9) = Quack, dans Janowski et Schwemer (éd.), Hymnen, Klagelieder und Gebete, 2013, p. 177 [5.6] ; Quack, dans Grund et al. (éd.), Festschrift für Bernd Janowski, 2013, p. 572-73 ; Barucq et Daumas, Hymnes et prières, 1980, p. 105-06 [19] ; Daressy, Ostraca, 1901, p. 41 [25209] et pl. XXXVII [25209] ; Erman, ZÄS 38 (1900), p. 30-33 ; TLA DZA 24.458.450, accessible à l'adresse http://aaew.bbaw. de/tla/ (consultée le 18/03/2019). Se référer aussi à la graphie de $m \underline{t} z$ avec le déterminatif divin présente sur un ostracon répertorié sur une fiche manuscrite du Wörterbuch: TLA DZA 24.458.500, accessible 
à l'adresse http://aaew.bbaw.de/tla/ (consultée le 18/03/2019).

${ }^{55}$ À corriger en faucon sur le pavois (Gardiner G7) d'après la photo de l'objet sur la planche de Daressy. Notons que la graphie hiératique du phallus est très proche de celle répertoriée par Möeller dans le papyrus Abbott (daté de Ramsès IX).

${ }^{56}$ Pap. Brooklyn 47.218.84, page $[\mathrm{X}+12,1]$ : « $(\mathrm{XI}, 11)(\ldots)$ Les êtres sont placés sur le djed, un vagin en métal près de lui, car les deux soeurs (XII,1) sont à proximité du phallus (...) »= Meeks, Mythes et Légendes du Delta, 2006, p. 25, 65-66 n. 105, 119 n. 382 et p. 480 pl. 12.

${ }^{57} \mathrm{La}$ permutation entre le déterminatif divin (dieu assis ou faucon sur le pavois : A40 ou G7) et celui de l'homme assis (A1) pour un même mot pouvant qualifier des entités de natures différentes suivant le contexte est bien connu dans la documentation égyptienne, notamment pour le mot $j t$ « père », renvoyant tantôt au père divin, tantôt au père humain : Wb I, 141, 10-13 ; AnLex 77.0493, 78.0535, 79.0366 ; TLA Lemma $\mathrm{n}^{\circ} 32820$ et les fiches manuscrites correspondantes (dernier accès le 11/04/2019); Lesko, $D L E^{2}, 2002$, p. 50 . Il en est de même pour le mot wtt $/ w t t w / w t \underline{t} w$ " géniteur, père » : VÉgA ID 6558 (consultable à l'adresse http://vegavocabulaire-egyptien-ancien.fr/acceder-a-loutil/, dernier accès le 11/04/2019); Wb I, 382, 10-12 ; AnLex 77.1097 ; TLA Lemma $\mathrm{n}^{\circ} 51310$ et les fiches manuscrites correspondantes (dernier accès le 11/04/2019) ; FCD, 1962, p. 72 ; Hannig, ÄWb, I, 2003, p. 387 \{8696\}; Hannig, $\ddot{A} W b, \mathrm{II} / 1,2006$, p. 745 \{8696\}.

${ }^{58}$ Pour d'autres cas dans lesquels le déterminatif de l'homme assis (A1) qualifie des éléments qui appartiennent au grand ensemble « être humain masculin », voir par exemple la graphie du mot jḥwn " enfant, adolescent, jeune homme » dans le papyrus Anastasi I, 1, 3 (pap. BM EA 10247 : lettre satirique envoyée par un scribe à un autre scribe). Dans cet exemple hiératique, le mot est, entre autres, déterminé par le signe A1 : VÉgA ID 7525 (dernier accès le 11/04/2019); Wb I, 122 ; Lesko, $D L E^{2}$, I, 2002, p. 44 [A1, 1, 3] ; Gardiner, Egyptian Hieratic Texts, 1911, p. 2a [1. 13]. Se référer également à la graphie du mot ‘ $d d$ « jeune homme, adolescent, serviteur » ainsi qu’à celle de son pendant féminin ‘ $\underline{d} d . t$ " jeune femme, adolescente » présentant respectivement, entre autres, les déterminatifs de l'homme assis (A1) et de la femme assise (B1). Pour ` $\underline{d} d$ : VÉgA ID 6773 (dernier accès le 11/04/2019); Wb I, 242, 11-17 ; AnLex 77.0797, 78.0842, 79.0574 ; Lesko, $D L E^{2}$, I, 2002, p. 84-85 ; TLA Lemma n 42290 (dernier accès le 11/04/2019); Wortdiskussionen, https://aegyptologie.philhist.unibas. ch/fileadmin/user_upload/aegyptologie/Forschung/ Werkzeuge/Wortdiskussionen/Wortdiskussionen_ Gesamtdokument.pdf, s.v. « ‘ $\underline{d} d$; ‘ $\underline{d} d$... ». Pour `d dd.t : VÉgA ID 6775 (dernier accès le 11/04/2019);Wb I, 242, 18-19 ; AnLex 79.0575 ; Lesko, DLE 2 I, 2002, p. 85 ; TLA Lemma $\mathrm{n}^{\circ} 42300$ (dernier accès le 11/04/2019). Sur ces deux termes, voir aussi Feucht, Das Kind im alten Ägypten, 1995, p. 515-18.

${ }^{59}$ Schneider, Asiatische Personennamen, 1992, p. 71-72 [N 129]; PN I, 1935-1977, p. 61 [6]. Sous cet anthroponyme, tous deux répertorient uniquement le vizir Âamtjou. Une troisième possibilité, permettant de concilier les deux points de vue, pourrait peutêtre être envisagée. Il s'agirait de considérer que c'est bien le signe $r$ qui est écrit, et non celui de l'oeuf, tout en étant suivi par le nom propre Âamtjou inscrit au moyen de la graphie pseudo-étymologique. La phrase serait alors : " [Sa Majesté a dit : “[J’]approuve grandement ${ }^{\mathbf{3}}$ Ouseramon plus que/à la place d'Âamtjou (i.e. "c'est considérable : (son) phallus"). [Depuis] combien de temps [...]" » ([Dd jn hmm=f: "jb=j] ${ }^{3} h n(r)$ Wsr-Jmn $r$ C $3 . t m \underline{t} 3(=f)$. Wr $n$ rnp [.wt $r$ $t 3 y]$ ” »). Cette solution ne résoudrait cependant pas entièrement les deux problèmes majeurs que sont le substantif féminin C $_{\text {. }} t$ ainsi que le sens produit par la para-étymologie ainsi créée afin de nommer Âamtjou.

${ }^{60}$ Comme suggéré par Federico Poole, communication personnelle.

${ }^{61}$ De notre point de vue, si la filiation directe entre Ouseramon et Âamtjou était effectivement exprimée ici, elle ne le serait uniquement qu'à travers une évocation de type sonore par le biais de la figure de style de l'assonance, jeu bien connu dans la littérature égyptienne et serait ainsi un marqueur supplémentaire de la qualité rédactionnelle de cette composition. Federico Poole nous fait cependant pertinemment remarquer que, dans le choix de traduction que nous privilégions, $3 . t$ et míz sont séparés non seulement comme mots mais qu'ils appartiennent également à deux phrases indépendantes séparées prosodiquement. Il ajoute que l'on peut même imaginer que le lecteur fasse une pause après '3.t avant de débuter la lecture de la phrase suivante commençant par $m \underline{t} 3$. L'hypothèse d'une résonnance exclusivement auditive qui aurait alors pour objectif d'évoquer en filigrane la légitimité héréditaire d'Ouseramon à exercer le vizirat pose donc problème et s'en trouve de fait fragilisée.

${ }^{62}$ Helck,Urk. IV, 1984² , 1384, 8 ; Helck, dans Firchow (éd.), Ägyptologische Studien, 1955, p. 112 fig. 3 ; Kruchten, Le grand texte oraculaire de Djéhoutymose, 1986, p. 43, 44 et n. 1 ; Černý, BIFAO 72 (1972), p. 67-68 [91-93] ; Théodoridès, RIDA 14 (1967), p. 112 et n. 3 ; Černý, BIFAO 41 (1942), p. 19-21 [36-37] ; Černý, BIFAO 35 (1935), p. 54-55.

${ }^{63}$ Stauder, Annuaire de l'EPHE, Section des sciences historiques et philologiques 147 (2016), http://ashp. revues.org/1799. Pour le signe šps, voir Möller II, 26.

${ }^{64} \mathrm{Je}$ remercie l'un de mes deux relecteurs anonymes pour cette suggestion de transcription.

${ }^{65}$ Voir sa restitution du nom du père d'Ouseramon dans sa traduction et ses notes de traduction mais pas dans sa transcription hiéroglyphique (Helck, dans Firchow [éd.], Ägyptologische Studien, 1955, p. 112, $115)$ et sa restitution de $\underline{d} d=f$ seulement dans sa transcription publiée dans les Urkunden (Helck, Urk. IV, $\left.1984^{2}, 1384,11\right)$.

${ }^{66}$ Stauder, dans Allen et al. (éd.), Coping with Obscurity, 2016, p. 169-99 ; Stauder, The Earlier Egyptian Passive, 2014, p. 324-34 ; Junge, Late Egyptian Grammar, 2001, p. 97-99 [§ 2.3.2 (1)], 131-131 [3.3.1 (3)] ; Winand, Études de néo-égyptien 1, 1992, p. 264-65 [§ 418-419] ; 
Silverman, dans Posener-Kriéger (éd.), Mélanges Gamal eddin Mokhtar, II, 1985, p. 276-77 ; Depuydt, OLP 14 (1983), p. 13-54 ; Polotsky, IOS 6 (1976), p. 14-25 ; de Cenival, RdE 24 (1972), p. 40-45 (en part. p. 41) ; Westendorf, ZÄS 84 (1959), p. 147-55 ; Gardiner, Egyptian Grammar, 1957³, § 129-30, 189, 1, 440, 1, 442, 3, 452, 4.

${ }^{67}$ Černý, Notebook, MSS 17.151, p. 35.

${ }^{68}$ h'y : Helck,Urk. IV, 1984², 1384, 13 = Helck, Urk. IV. Übersetzung, 1961, p. 71 (Es glänzen die Augen); Helck, dans Firchow (éd.), Ägyptologische Studien, 1955, p. 112 fig. 3, 115 (Es glänzen seine Augen); suivi par Cumming, Egyptian Historical Records, II, 1984, p. 97 (He has been found as a man of whom men say, 'The eyes shine being full of ideas...) et Dziobek, Denkmäler Vezirs User-Amun, 1998, p. 99 (es erstrahlen [seine] Augen);Wb III, 239, 4-241, 2 ; AnLex 77.3008, 78.2957, 79.2158.

${ }^{69} \mathrm{~Wb} \mathrm{~V}, 636,1-2$; AnLex 77.5300 ; TLA Lemma ${ }^{\circ}$ 186360 (geblendet sein [von den Augen], durchbohren [mit den Augen]) et DZA 31.879.390 et 31.879 .400 (dernier accès le 03/02/2018) ; Devaud, Kêmi 2 (1929), p. 17 [14].

${ }^{70}$ Wente, Letters from Ancient Egypt, 1990, p. 98-110 ; Fischer-Elfert, Die satirische Streitschrift des Papyrus Anastasi I, 1986, p. 95, 98 ; Gardiner, Egyptian Hieratic Texts, 1911, p. 14* [VIII.], 20 ; Birch, Select Papyri, I/2, 1842, pl. XLV.

${ }^{71}$ Caminos, LEM, 1954, p. 381-83 ; Gardiner, LEM, 1937 , p. 102 (10-11) ; Blackman et Peet, JEA 11 (1925), p. 287 et n. 14 ; Erman et Lange, Papyrus Lansing, 1925, p. 20-21, 48 ; Budge, Hieratic Papyri in the British Museum, 1923, pl. 17-18.

${ }^{72}$ Demarée, The Bankes Late Ramesside Papyri, 2006, p. 10-11 et pl. 7 ; Wente, Letters from Ancient Egypt, 1990, p. 203 [330] ; Edwards, JEA 68 (1982), p. 132-33 et n. a et pl. XIII. Consulter également l'édition d'I. Hafemann, accessible en ligne sur le TLA : http://aaew.bbaw.de/tla/servlet/ GetTextDetails? $\mathrm{u}=$ guest $\& \mathrm{f}=0 \& \mathrm{l}=0$ \& $\mathrm{tc}=18953 \& \mathrm{db}=0$ (dernière consultation le 09/04/2019).

${ }^{73}$ Vernus, Sagesses de l'Égypte pharaonique, $2010^{2}$, p. 44152 (notamment p. 448 et n. 31 " un éblouissement des pupilles, un éblouissement des yeux »); Quack, dans Hoffmann et Quack (éd.), Anthologie der demotischen Literatur, 2007, p. 237, 360-61 ; Winand, ChronEg LXXIII/145 (1998), p. 42-53 (notamment p. 52 [17] « regard étincelant »); Quack, WdO 24 (1993), p. 17 et n. 74 ; Jasnow, A Late Period Hieratic Wisdom Text, 1992, p. 96 [17.], 109 [5/17, n. FF] et fig. 10-11.

${ }^{74}$ Pour hn « discours, paroles, affaire, plainte, pétition, savoir cognitif » : Wb III, 289, 1-14 ; AnLex 77.3090, 78.3034, 79.2216 ; TLA Lemma no 117520 (dernier accès le 04/03/2018) ; Wortdiskussionen, https://aegyptologie.philhist.unibas.ch/ fileadmin/user_upload/aegyptologie/Forschung/ Werkzeuge/Wortdiskussionen/Wortdiskussionen Gesamtdokument.pdf, s.v. « hn »; FCD, 1962, p. 192 ; Ockinga, JEA 69 (1983), p. 89-90.

${ }^{75}$ Helck,Urk. IV, 1984² 1384,14 ; Helck, dans Firchow (éd.), Ägyptologische Studien, 1955, p. 112 fig. 3, 115.

${ }^{76}$ Helck, dans Firchow (éd.), Ägyptologische Studien, 1955, p. 115 ; Stauder, Annuaire de l'EPHE, Section des sciences historiques et philologiques 147 (2016), http:// ashp.revues.org/1799.

${ }^{77}$ Helck, Urk. IV, 1984² 1384,18 ; Helck, dans Firchow (éd.), Ägyptologische Studien, 1955, p. 112 fig. 3, 115 ; Černý, Notebook MSS 17.151, p. 35. L'ostracon CGC 25339 mentionne le vizir de Ramsès II Thoutmose, daté de la fin de son règne (entre l'an 21 et l'an 30 ?) : Raedler, dans Gundlach et Klug (éd.), Das ägyptische Königtum, 2004, p. 292 et n. 111, p. 297 ; Helck, Verwaltung, 1958, p. 320-21, 456 [31,b] ; Weil, Veziere, 1908, p. 119 [§ 45] ; Daressy, Ostraca, 1901, p. 87-88 et pl. LX.

${ }^{78}$ Helck, dans Firchow (éd.), Ägyptologische Studien, 1955, p. 115 ; Sethe, Urk. IV, $1961^{3}$, 1076, 14-16 (Rekhmirê) ; Sethe, Urk. IV, 19613, 968, 8-9 (Antef).

${ }^{79}$ Kruchten, Les annales des prêtres de Karnak (XXIXXIII $^{\text {mes }}$ dynasties), 1989, p. 12-23.

${ }^{80}$ En suivant, selon toute vraisemblance, la note de traduction et la restitution de Helck (Helck, Urk. IV, 1984² , 1384, 5 ; Helck, dans Firchow [éd.], Ägyptologische Studien, 1955, p. 112 fig. 3, 114).

${ }^{81}$ Signalons que nous retrouvons un exemple du même type de question-réponse postérieurement dans Le voyage d'Ounamon : wr $r$ p 3 hrw $m-\underline{d} r j w=k n$ p $n t j$ Jmn jm ? 5 sbd hrw r pзjj « Combien de jours jusqu’à maintenant depuis que tu es venu de la place où est Amon ? 5 mois entiers jusqu’à maintenant » : Gardiner, LES, 1932, p. 66, 7-9.

${ }^{82}$ Winand, SAK 13 (1986), p. 306-7. Pour les traces de néo-égyptien présentes dans notre texte, voir Neveu, La langue des Ramsès, 1996, p. 4-5, 10-11, 13 ; Černý, JEA 23 (1937), p. 57-59.

${ }^{83}$ Chantrain et Winand, $R d E 63$ (2012), p. 43-66 ; Oréal, Les particules en égyptien ancien, 2011, p. 437-86.

${ }^{84}$ Comparez avec la description des trois qualités éminentes du canditat dans la Nomination (TT 131) : $h m w$-jb htp $p(w) q d$-nfr « c'est quelqu'un à l'esprit expert, quelqu'un d'apaisé, quelqu'un à l'excellent caractère » (col. 25, $2^{\mathrm{e}}$ discours des courtisans = Dziobek, Denkmäler Vezirs User-Amun, 1998, pl. I, col. 25).

${ }^{85}$ Dziobek, Denkmäler Vezirs User-Amun, 1998, p. 3-21 et pl. 1 ; Dziobek, Gräber Vezirs User-Amun, 1994, p. 7375 et pl. 17a, 42-43, 72, 81 [scène 131-5] ; Helck, Urk. IV, 1984² 1380-83 [420] = Helck, Urk. IV. Übersetzung, 1961, p. 70-71 [420] ; $\mathrm{PM} \mathrm{I}^{2} / 1,1960^{2}$, p. 246 [8].

${ }^{86}$ Helck, dans Firchow (éd.), Ägyptologische Studien, 1955, p. 107-17.

${ }^{87}$ Stauder, dans Vuilleumier et Meyrat (éd.), Mélanges pour une collègue distinguée, (à paraître), § 8 ; Gnirs, dans Bickel (éd.), Studien zum historischen Bewusstsein in der Thutmosidenzeit, 2013, p. 138-43. Sur la Königsnovelle d'une manière plus générale, lire entre autres Loprieno, dans Loprieno (éd.), Ancient Egyptian Literature, 1996, p. 277-95.

${ }^{88}$ Vernus, Sagesses de l'Égypte ancienne, 2010², p. 59-62, 71-72 ; Dziobek, Denkmäler Vezirs User-Amun, 1998, p. 23-54 et pl. 2 ; Dziobek, Gräber Vezirs User-Amun, 1994, p. 75-77 [scène 131-6] et pl. 18-19, 72, 82 ; PM $\mathrm{I} / 1^{2}, 1960^{2}$, p. 246 [9.II].

${ }^{89}$ Dziobek, Denkmäler Vezirs User-Amun, 1998, p. 55-66 et pl. III a-b ; Dziobek, Gräber Vezirs User-Amun, 1994, p. 77 [scène 131-8] et pl. 17b-c, 74, 84 ; Lichtheim, 
Ancient Egyptian Literature, II, 1976, p. 21-24 ; Faulkner, JEA 41 (1955), p. 18-29; PM I/12 $1960^{2}$, p. 209 [5] (R) ; PM I/12 $1960^{2}$, p. 246 [12] (W) ; PM $\mathrm{I} / 1^{2}, 1960^{2}$, p. 132 [6] (H) ; Davies, Tomb of Rekh-mi$R \bar{e} e, 1943$, p. 15-17, 84-88 et pl. XIII-XV, CXVI-CXVIII (synopses des versions de Rekhmirê, d'Ouseramon et d'Hapou).

${ }^{90}$ Tallet, dans Warmenbol et Angenot (éd.), Thèbes aux 101 portes, 2010, p. 153-63 ; Tallet, ChronEg LXXX/159 (2005), p. 66-75; Quirke, Titles and Bureaux, 2004, p. 18-23 ; Lorton, dans Bryan et Lorton (éd.), Essays in Egyptology, 1994, p. 147-55 ; Van Den Boorn, Duties of the Vizier, 1988 ; Davies, Tomb of Rekh-mi-Rēe, 1943, pl. XXVI-XXVIII, CXIX-CXXII (synopses des versions de Rekhmirê, d'Ouseramon, d'Aménémopé et de Paser).

${ }^{91}$ Davies, Tomb of Rekh-mi-Rée, 1943, pl. XXIV.

${ }^{92}$ Sethe, Urk. IV, 196033, 1071-85 [325] ; Davies, Tomb of Rekh-mi-Rēe, 1943, pl. XI-XII ; Gardiner, ZÄS 60 (1925), p. 62-76.

${ }^{93}$ Cardin et Goyon (dir.), Trésors d'Égypte, 2004, p. 24-29 [HC 1]; Dziobek, Denkmäler Vezirs User-Amun, 1998, p. 88-91 et pl. 12 ; Eichler, SAK 25 (1998), p. 62 ; Kruchten, Les annales des prêtres de Karnak (XXIXXIII ${ }^{\text {mes }}$ dynasties), 1989, p. 188-89 ; Sethe, Urk. IV, $1960^{3}, 1031,15-16$

${ }^{94}$ Kruchten, Les annales des prêtres de Karnak (XXI-XXIII ${ }^{\text {mes }}$ dynasties), 1989, p. 16-18, 147-204 (en particulier p. 147-49, 161-62, 186-92). Voir plus récemment Quack, SAK 36 (2007), p. 249-95 ; Quack, dans Assmann et Bommas (éd.), Ägyptische Mysterien?, 2002, p. 95-108.

${ }^{95}$ VÉgA ID 5282 ; Wb II, 494, 10-495, 12 ; AnLex 77.2510, 78.2504, 79.1833; Wortdiskussionen, https://aegyptologie.philhist.unibas.ch/ fileadmin/user_upload/aegyptologie/Forschung/ Werkzeuge/Wortdiskussionen/Wortdiskussionen Gesamtdokument.pdf, s.v. «hnn»; FCD, 1962, p. 159.

${ }^{96}$ « Faire une inclinaison de la tête, approuver ». Voir notamment Kruchten, Le grand texte oraculaire de Djéhoutymose, 1986, p. 46 et n. 5, 47 et n. 1-3, 105 et n. 1 ; Kákosy, dans Helck et Westendorf (éd.), LÄ IV, 1982, col. 600-6, s.v. « Orakel »; Vernus, CahKarn 6 (1973-1977), p. 229 (ar) ; Černý, dans Parker, A Saite Oracle Papyrus, 1962, p. 35-48 (notamment p. 44-45) ; Černý, BIFAO 35 (1935), p. 56-57 ; Černý, BIFAO 30 (1930), p. 491-96.

${ }^{97}$ Statue CGC 42185, inscription I, $b$ (côté gauche de la statue) : KRI IV, 1982, 129-131 = KRITA IV, 2003, p. 98-99 = KRITANC IV, 2014, p. 112-13.

${ }^{98}$ Černý, Notebook MSS 17.151, p. 35 ; Faulkner, JEA 41 (1955), p. 19 fig. 1 (1. 2) ; Davies, Tomb of Rekh$m i-R \bar{e} e, 1943, \mathrm{pl}$. XIV. Pour $d h n$ " promouvoir, désigner, nommer, mettre en place, insérer dans un bureau/une fonction " : Wb V, 479, 6-21; AnLex 77.5082, 79.3581; Wortdiskussionen, https://aegyptologie.philhist.unibas.ch/ fileadmin/user_upload/aegyptologie/Forschung/ Werkzeuge/Wortdiskussionen/Wortdiskussionen_ Gesamtdokument.pdf, s.v. «dhn »; TLA Lemma $\mathrm{n}^{\circ}$ 180290 (dernier accès le 04/03/2018) ; FCD, 1962, p. 315.

${ }^{99}$ Dziobek, Gräber Vezirs User-Amun, 1994, pl. 84 ; Davies,
Tomb of Rekh-mi-Rée, 1943 , pl. CXVI.

${ }^{100}$ Helck, Urk. IV, $1984^{2}$, 1385-90 ; PM I/1² $1960^{2}$, p. 192

(17) ; Davies, The Tomb of Ken-Amūn at Thebes, I, 1930, p. 17-19 et pl. VIII.

${ }^{101}$ Willems, dans Flossmann-Schütze et al. (éd.), Kleine Götter - Grosse Götter, 2013, p. 553-58 ; Lloyd, dans Lloyd (éd.), Studies in Pharaonic Religion and Society, 1992, p. 24 (2cIX) ; Sethe, Urk. VII, 1935, 34, 1 ; Newberry et Griffith, Beni Hassan, I, 1893, p. 65 [7] et pl. XXVI, col. 184-186.

${ }^{102}$ Willems, dans Flossmann-Schütze et al. (éd.), Kleine Götter - Grosse Götter, 2013, p. 553-58 ; Zonhoven, ZÄS 125 (1998), p. 84-85 (§ 5); Derchain, RdE 43 (1992), p. 35-47 ; Goedicke, dans Anonyme (éd.), Festschrift zum 150 jährigen Bestehen des Berliner Ägyptischen Museums, 1974, p. 87, 94 (r) et pl. 8 ; de Buck, dans Blackman et al. (éd.), Studia Aegyptiaca, I, 1938, p. 49 (1. 9-10). Attention cependant : mts désignerait peut-être ici un certain type de tissu. Pour la datation du texte, voir en dernier lieu Stauder, Linguistic Dating of Middle Egyptian Literary Text, 2013, p. 249-57.

${ }^{103}$ Obsomer, Sésostris $I^{\text {er }}$, 1995, p. 676-81 [137] (et bibliographie antérieure) ; Smith, The Fortress of Buhen, 1976, p. 39-41 et pl. LXIX.1 ; Bosticco, Museo Archeologico di Firenze, I, 1959, p. 31-33 [29] et pl. 29b.

${ }^{104}$ Dziobek, Denkmäler Vezirs User-Amun, 1998, p. 99 , 101 ; Feucht, Das Kind im Alten Ägypten, 1995, p. 248 ; Janssen et Janssen, Growing Up and Getting Old in Ancient Egypt, 1990, p. 94 ; Westendorf, dans Helck et Otto (éd.), LÄ I, 1975, col. 728, s.v. " Beschneidung »; Helck, dans Firchow (éd.), Ägyptologische Studien, 1955, p. 115. Contra : Quack, dans Berlejung et al. (éd.), Menschenbilder und Körperkonzepte, 2012, p. 595-96.

${ }^{105}$ Willems, dans Flossmann-Schütze et al. (éd.), Kleine Götter - Grosse Götter, 2013, p. 553-58; Quack, dans Berlejung et al. (éd.), Menschenbilder und Körperkonzepte, 2012, p. 561-51 ; Megahed et Vymazalovà, Anthropologia 49/2 (2011), p. 155-64 ; Spigelman, BACE 8 (1997), p. 91-100 ; Bailey, BACE 7 (1996), p. 15-28 ; Feucht, Das Kind im Alten Ägypten, 1995, p. 245-55 ; Westendorf, dans Helck et Otto (éd.), LÄ I, 1975, col. 727-29, s.v. « Beschneidung »; de Wit, ZÄS 99 (1972), p. 41-48; Jonckheere, Centaurus 1/3 (1951), p. 212-34.

${ }^{106}$ Dziobek, Denkmäler Vezirs User-Amun, 1998, p. 163-64.

${ }^{107}$ Afin de prolonger l'explication de ce passage, mentionnons, en dernière hypothèse, qu'en prenant en compte le sens plus abstrait de valeur d'échange qu'a l'argent, la phrase pourrait peut-être aussi se comprendre de cette manière : le vizir est quelqu'un qui accorde plus d'échange que le roi, il est plus accessible que le roi. Cette formulation viendrait donc illustrer le rôle d'intermédiaire qu'accomplit le vizir. La fragilité de l'argument rend cependant cette proposition d'interprétation discutable en l'état actuel de conservation du texte.

${ }^{108}$ Helck, dans Firchow (éd.), Ägyptologische Studien, 1955, p. 115.

${ }^{109} \mathrm{~Wb}$ III, 244, 10-245, 22 ; AnLex 77.3020, 78.2961, 79.2166; Wortdiskussionen, https:// aegyptologie.philhist.unibas.ch/fileadmin/ 
user_upload/aegyptologie/Forschung/

Werkzeuge/Wortdiskussionen/Wortdiskussionen_ Gesamtdokument.pdf, s.v. « hww »; Grandet, DiscEg 8 (1987), p. 89-90, n. 17 (pour la bibliographie supplémentaire). Pour hw Km.t/tz.wy voir notamment Galán, JNES 59/4 (2000), p. 255-64 (en particulier p. 260-61 et n. 39); Grimal, Les termes de la propagande royale égyptienne, 1986, p. 75, 328-29. Voir quelques exemples d'emplois de $h w$ dans la stèle de Kouban (1. 2 = KRI II, 1979, 354, 2 [126] : hw $\sim n=f$ Km.t $m \underline{d} n h=f)$, dans la version d'Abou Simbel de la stèle du premier mariage hittite (1. 12-13 = KRI II, 1979, 238, 3 [66] : hw Km.t $m k j d b$. wy jr t3̌s=f $r \quad m r r=f)$ ainsi que dans la stèle Ismaïlia 2758 (côté gauche, 1.3 = KRI II, 1979, 304, $3: \underline{h}=\underline{t} m k 3$ nrp r hww Km.t).

${ }^{110}$ VÉgA ID 1183, s.v. « $n b w »$; Ogden, dans Nicholson et Shaw (éd.), Ancient Egyptian Materials and Technology, 2000, p. 161-66 ; Aufrère, L'univers minéral, II, 1991, p. 367-74 (et plus généralement le chapitre consacré à « la théologie de l'or et des divers alliages aurifères » p. 353-406. Sur la nature des dieux et du souverain sous leur aspect minéral, voir plus précisément Aufrère, L'univers minéral, I, 1991, p. 311-19) ; Grimal, Les termes de la propagande royale, 1986, p. 126-28; Coche-Zivie, BIFAO 74 (1974), p. 105-9 ; Grapow, Die bildliche Ausdrücke des Aegyptischen, 1924, p. 53, 5758. Plus généralement, sur les métaphores et figures de styles égyptiennes, voir Guglielmi, dans Loprieno (éd.), Ancient Egyptian Literature, 1996, p. 465-97 ; Brunner, dans Helck et Otto (éd.), LÄ I, 1975, col. 80511, s.v. " Bildliche Ausdrücke und Übertragungen »; Osing, dans Helck et Westendorf (éd.), LÄ II, 1977, col. 618-24, s.v. « Gleichnis »; Guglielmi, dans Helck et Westendorf (éd.), L $\ddot{A}$ VI, 1986, col. 22-41, s.v. " Stilmittel »; Guglielmi, dans Helck et Westendorf (éd.), LÄ VI, 1986, col. 986-89, s.v. « Vergleich ». Voir aussi la toute récente publication de la thèse de doctorat de Hsu, Bilder für den Pharao, 2017.

${ }^{111}$ Stèle de la Tempête, $\mathrm{r}^{\circ} 12=\mathrm{V}^{0} 14$ : Biston-Moulin, CahKarn 15 (2015), p. 46-49 (§ 2); Beylage, Aufbau der königlichen Stelentexte, 2002, p. 77-85 (notamment p. 83) ; Klug, Königliche Stelen, 2002, p. 35-46 (notamment p. 40) et p. 501-2 (bibliographie); Ritner et Moeller, JNES 73/1 (2014), p. 7 ; Allen et Wiener, JNES 57/1 (1998), p. 3-5, 14, 20 ; Helck, Historischbiographische Texte, 1975, p. 108 [124] ; Vandersleyen, RdE 19 (1967), p. 125.

${ }^{112} \mathrm{~Wb}$ V, 538, 20 ; AnLex 77.5170, s.v. « $\underline{d} \mathrm{c} m n(y)$ nsw.wt » et $W b \mathrm{~V}, 544,18$; AnLex 77.5172, 78.4888, s.v. « $\underline{d} w$ $n(y) n b w »$.

${ }^{113}$ Schiff Giorgini, Soleb V, 1998, pl. 302-303.

${ }^{114}$ Brunner, Die südlichen Räume des Tempels von Luxor, 1977, p. 70, fig. 21.

${ }^{115}$ Helck, Urk. IV, 1984² 1658,13 [563] et Helck, Urk. IV, $1984^{2}, 1961,11$ [744].

${ }^{116} \mathrm{KRI}$ II, 1979, 433, 11 [158.B.4] ; Coche-Zivie, BIFAO 74 (1974), p. 105 ; AnLex 79.3632.

${ }^{117}$ The Epigraphic Survey, Medinet Habu, VIII, 1970, p. 12 et pl. 623.

${ }^{118}$ KIU 1730 (pour « Karnak Identifiant Unique » qui permet d'accéder aux notices complètes des documents de Karnak [textes hiéroglyphiques, photographies, bibliographie, etc.] dans le projet Karnak, CNRS, USR 3172-CFEETK/UMR 5140Univ. Montpellier, Équipe ENiM - Programme « Investissement d'Avenir » ANR-11-LABX-0032-01 LabEx Archimede) à l'adresse http://sith.huma-num. fr/karnak - consultée le 20/03/2018 ; Sethe, Urk. IV, $1961^{3}, 362,8$.

${ }^{119}$ Statue BM 48, 1.10 (= Salt 1829): $\mathrm{PM} \mathrm{II}^{2}, 1972^{2}$, p. 454 ; Sethe, Urk. IV, $1961^{3}, 546,11$; Edwards, Hieroglyphic Texts, 1939, p. 5 et pl. V. Statue CGC 1013 : Borchardt, Statuen und Statuetten, IV, 1934, p. 26.

${ }^{120}$ Binder, The Gold of Honour, 2008, exergue et p. 286 [004] ; Murnane, Texts from the Amarna Period, 1995, p. 116 [58-B.5] ; Helck, Urk. IV, 1984², 2000, 8 = Helck, Urk. IV. Übersetzung, 1961, p. 352 ; Sandman, Texts from the Time of Akhenaten, 1938, p. 96 ; Davies, The Rock Tombs of el Amarna, VI, 1908, p. 23 et pl. XXX.

${ }^{121}$ Ogden, dans Nicholson et Shaw (éd.), Ancient Egyptian Materials and Technology, 2000, p. 170-71 ; Schwarz, ZÄS 123 (1996), p. 69-84 ; Aufrère, L'univers minéral, II, 1991, p. 407-28 (chapitre consacré à la « théologie de l'argent »); Grapow, Die bildliche Ausdrücke des Aegyptischen, 1924, p. 57-58.

${ }^{122}$ Hornung, Der ägyptische Mythos von der Himmelskuh, $1991^{2}$, p. 1, 37,5, 52 n. 6 ; Maystre, BIFAO 40 (1941), p. 53-115.

${ }^{123}$ Vandier, Le papyrus Jumilhac, 1961, p. 124, 180-81 [370].

${ }^{124}$ Faulkner, JEA 22 (1936), p. 131 ; Faulkner, The Papyrus Bremner-Rhind, 1933, p. 29, 6.

${ }^{125} \mathrm{KRI}$ II, 1979, 233 [66] (versions connues du texte), 237, 14-15; Kuentz, ASAE 25 (1925), p. 197, 228.

${ }^{126}$ Gale et Stos-Gale, JEA 67 (1981), p. 103-15 ; Lucas, Ancient Egyptian Materials and Industries, 1962, p. 490-92 ; Harris, Lexicographical Studies in Ancient Egyptian Minerals, 1961, p. 32-50 ; Lucas, JEA 14 (1928), p. 313-19.

${ }^{127}$ Yoyotte, ChronEg XXVIII/55 (1953), p. 34-35 ; Leclant, $R d E 8$ (1951), p. 104-13 et fig. 5 et pl. 4.

${ }^{128}$ Lucas, Ancient Egyptian Materials and Industries, 1962 p. 252 ; Lucas, dans Carter, The Tomb of Tut-ankhAmen, III, 1933, p. 173-75 ; Lucas, dans Carter, The Tomb of Tut-ankh-Amen, II, 1927, p. 174-75.

${ }^{129}$ Davies, Tomb of Rekh-mi-Rée, 1943, pl. XIV. La version de l'Installation dans la tombe d'Ouseramon (TT 131) est en lacune à cet endroit (Dziobek, Denkmäler Vezirs User-Amun, 1998, pl. 3). Voir cependant la planche synoptique de Davis (Davies, Tomb of Rekh-mi-Rée, 1943, pl. CXVI) qui a copié une partie de ce passage. Sur le métal-ḥmtj et son utilisation métaphorique, notamment du roi comme rempart, voir $\mathrm{Wb}$ III, 99 et Wb I, 436-438; Aufrère, L'univers minéral, II, 1991, p. 438, 449-57 (chapitre consacré au « métaux cuivreux, plomb et étain ", notamment p. 450); Grimal, Les termes de la propagande royale, 1986, p. 331-35 ; Græfe, Untersuchungen zur Wortfamiliebj3, 1971 ; Grapow, Die bildliche Ausdrücke des Aegyptischen, 1924, p. 59, 163-64.

${ }^{130}$ Tablette hiératique DeB, $\mathrm{v}^{\mathrm{o}} \mathrm{II}=$ Vernus, $R d E 33$ (1981), p. 89, n. 1, p. 107-8 et p. 110, n. a, p. 114, n. v, x ; Laboury, La statuaire de Thoutmosis III, 1998, p. 18. ${ }^{131}$ Shirley, dans Galán et al. (éd.), Creativity and 
Innovation in the Reign of Hatshepsut, 2014, p. 173-245 (notamment p. 176, 184-86) ; Bryan, dans Cline et O'Connor (éd.), Thutmose III, 2006, p. 69-77 ; Dorman, dans Cline et O'Connor (éd.), Thutmose III, 2006, p. 39-58 ; Dziobek, dans Assmann et al. (éd.), Thebanische Beamtennekropolen, 1995, p. 129-40.

${ }^{132}$ Dorman, dans Cline et O’Connor (éd.), Thutmose III, 2006, p. 46 ; Dziobek, Denkmäler Vezirs User-Amun, 1998, p. 98 ; Dorman, The Monuments of Senenmut, 1988, p. 33-34, n. 81.

${ }^{133}$ Megally, Les variations et la transformation des formes hiératiques, 1971, p. XVII-XXII. Se référer également à Davies et Laboury (éd.), Oxford Handbook of Egyptian Epigraphy and Palaeography, 2019.

${ }^{134}$ Schweitzer, dans Moers et al. (éd.), Dating Egyptian Literary Texts, 2013, p. 177-90 ; Quack, dans Moers et al. (éd.), Dating Egyptian Literary Texts, 2013, p. 405-69 ; Stauder, Linguistic Dating of Middle Egyptian Literary Text, 2013, en part. p. 57-68, 136-55, 499-522.

${ }^{135}$ Dresbach, Das Wesirat, 2012, p. 69-72 ; Helck, Verwaltung, 1958, p. 460-64.

${ }^{136}$ Dorman, The Monuments of Senenmut, 1988, p. 33-34, 44.

${ }^{137}$ Loprieno, dans Loprieno (éd.), Ancient Egyptian Literature, 1996, p. 515-29.

${ }^{138}$ À titre de comparaison temporelle avec l'Histoire de France, c'est comme si une élaboration littéraire faisait actuellement référence (en 2019) à l'un des (premiers) ministres en exercice entre la $56^{\mathrm{e}}$ année du règne de Louis XIV (1699) et la $44^{\mathrm{e}}$ année du règne de Louis XV (1759) environ.

${ }^{139} \mathrm{KRI}$ I, 1969, 336, 2-4 = KRITA I, 1993, p. 273 ; PM I², $1960^{2}$, p. 97 [4] ; Helck, Verwaltung, 1958, p. 285, 433 [2] ; Davies, Two Ramesside Tombs, 1927, p. 20-22 et pl. XV.

${ }^{140}$ Mathieu, dans Zivie-Coche et Guermeur (éd.), "Parcourir l'éternité", 2012, p. 819-52 (notamment p. 823-24 et bibliographie antérieure p. 819, n. 1) ; KRI III, 1980, 492, 14-494, 6 = KRITA III, 2000, p. 349-50 [221] = KRITANC III, 2013, p. 358-61 [221] ; PM III $/ 2^{2}$, 1974², p. 571-72.

${ }^{141}$ Dziobek, Gräber Vezirs User-Amun, 1994 ; Dziobek, MDAIK 45 (1989), p. 109-32.

${ }^{142}$ Maruéjol, Thoutmosis III, 20142, p. 137-46, 204 ; Grandet, Les pharaons du Nouvel Empire, 2008, p. 81-94, 295-303 ; Redford, The Wars in Syria and Palestine of Thutmose III, 2003, p. 3-43 et fig. 1-4 ; PM $\mathrm{II}^{2}, 1972^{2}$, p. $97-98$ (280-282) ; Sethe, Urk. IV, $1961^{3}$, p. 647-75.

${ }^{143}$ Manassa, Imagining the Past, 2013, p. 66-101, 177 86 (Prise de Joppé) et 102-16, 187-94 (Thoutmosis III en Asie) ; Gnirs et Loprieno, dans Gundlach et Vogel (éd.), Militärgeschichte des pharaonischen Ägypten, 2008, p. 243-308 ; Spalinger, The Transformation of an Ancient Egyptian Narrative, 2002, en part. p. 317-46 et p. 347-65. À cela peut être ajouté La Querelle d'Apophis et Seqenenrê (papyrus Sallier I = pap. BM EA 10185) qui illustre, de manière plus générale, les productions littéraires ramessides d'une certaine mémoire de l'histoire. Ces textes sont néanmoins quelque peu différents de celui du papyrus Turin Cat. 1878 v ${ }^{\circ}, 1.1-9$ puisqu'ils apparaissent dans un contexte de guerres à l'étranger et développent un registre martial, alors que notre papyrus se situe, quant à lui, dans un contexte de nomination d'un individu.

${ }^{144}$ En dernier lieu, voir notamment Collombert, dans Charron et Barbotin (dir.), Savoir et pouvoir à l'époque de Ramsès II, 2016, p. 42-43 ; Snape, dans Snape et Collier (éd.), Ramesside Studies in Honour of K.A. Kitchen, 2011, p. 465-73.

${ }^{145}$ Pour le vizir Paser, voir notamment Obsomer, Ramsès II, 2012, p. 286-91 ; Raedler, dans Gundlach et Klug (éd.), Das ägyptische Königtum, 2004, p. 309-54 ; PM $\mathrm{I} / 1^{2}, 1960^{2}$, p. 221 [8].

${ }^{146}$ Stauder, Linguistic Dating of Middle Egyptian Literary Texts, 2013, p. 215-36 (§ 3.4); Van der Plas, dans Moers et al. (éd.), Dating Egyptian Literary Texts, 2013, p. 471-82 ; Hagen, JARCE 49 (2013), p. 77-79, 91 ; Van der Plas, GöttMisz 73 (1984), p. 51-52.

${ }^{147}$ Sur ce sujet, consulter par exemple Manassa, Imagining the Past, 2013, p. 143-63 ; Gnirs, dans Moers et al. (éd.), Dating Egyptian Literary Texts, 2013, p. 367-403 (part. p. 372-76, 384-94); Gnirs, dans Bickel (éd.), Studien zum historischen Bewusstsein in der Thutmosidenzeit, 2013, p. 127-86 ; FischerElfert, dans Tait (éd.), Egypt's View of its Past, 2003, p. 119-37 ; Manassa, dans Melville et Slotsky (éd.), Opening the Tablet Box, 2010, p. 245-69 ; Moers, dans Assmann et Blumenthal (éd.), Literatur und Politik im pharaonischen und ptolemäischen Ägypten, 1999, p. 37-52 ; Eyre, dans Loprieno (éd.), Ancient Egyptian Literature, 1996, p. 415-33 ; Baines, dans Loprieno (éd.), Ancient Egyptian Literature, 1996, p. 157-74 (part. p. 166-67).

${ }^{148}$ Assmann, La mémoire culturelle, 2010, (en part. p. 29-43); Baines, dans Baines, Visual and Written Culture in Ancient Egypt, 2007, p. 179-201 ; Vernus, Essai sur la conscience de l'histoire, 1995 ; McDowell, dans Demarée et Egberts (éd.), Village Voices, 1992, p. 95-109.

\section{Bibliographie}

Al. Ayedi, A.B., Index of Egyptian Administrative, Religious and Military Titles of the New Kingdom, Ismailia 2006.

Allen, J.P. et M.H. Wiener, « Separate Lives: The Ahmose Stela and the Theran Eruption », JNES 57/1 (1998), p. 1-28.

Allon, N. et H. Navratilova, Ancient Egyptian Scribes. A Cultural Exploration, London 2007.

AnLex = Meeks, D., Année lexicographique I-III, Paris $1998^{2}$.

Anonyme, dans W. Helck et W. Westendorf (éd.), LÄ IV, 1982, col. 732-44, s.v. « Papyri Turin ».

Assmann, J., La mémoire culturelle. Écriture, souvenir et imaginaire politique dans les civilisations antiques, Paris 2010.

Assmann, J., dans W. Helck et W. Westendorf (éd.), LÄ IV, 1982, col. 489-96, s.v. « Nilhymnus ».

Auenmüller, J.S.G., « Die Territorialität der Ägyptischen Elite(n) des Neuen Reiches » (thèse de doctorat, Freien Universität Berlin), Berlin 2013.

Aufrère, S., L'univers minéral dans la pensée égyptienne, I-II (BiEtud 105), Le Caire 1991.

Bacchi, B., L'Inno al Nilo (PERMT 4), [s.d]. 
Bailey, E., « Circumcision in Ancient Egypt », BACE 7 (1996), p. 15-28.

Baines, J., « Ancient Egyptian Concepts and Uses of the Past: Third to Second Millennium Evidence », dans J. Baines, Visual and Written Culture in Ancient Egypt, Oxford 2007, p. 179-201.

Baines, J., " Classicism and Modernism in the Literature of the New Kingdom », dans A. Loprieno (éd.), Ancient Egyptian Literature. History and Forms (ProblÄg 10), Leiden 1996, p. 157-74.

Baines, J., " Orality and Literacy », dans J. Baines, Visual and Written Culture in Ancient Egypt, Oxford 2007, p. 146-78.

Barucq, A. et Fr. Daumas, Hymnes et prières de l'Égypte ancienne (LAPO 10), Paris 1980.

Bellion, M., Égypte ancienne : catalogue des manuscrits hiéroglyphiques et hiératiques et des dessins, sur papyrus, cuir ou tissu, publiés ou signalés, Paris 1987.

Beylage, P., Aufbau der königlichen Stelentexte vom Beginn der 18. Dynastie bis zur Amarnazeit (̈̈AT 54), Wiesbaden 2002.

Binder, S., The Gold of Honour in New Kingdom Egypt (ACE-Stud. 8), Sydney 2008.

Biston-Moulin, S., «À propos de deux documents d'Ahmosis à Karnak. Karnak Varia (§ 1-2) ", CahKarn 15 (2015), p. 23-38.

Birch, S., Select Papyri in the Hieratic Character from the Collection of the British Museum, I/2, London 1842.

Blackman, A.M. et T.E. Peet, « Papyrus Lansing: A Translation with Notes », JEA 11 (1925), p. 284-98.

Boorn, G.P.F. van den, The Duties of the Vizier. Civil Administration in the Early New Kingdom, London New York 1988.

Borchardt, L., Statuen und Statuetten von Königen und Privatleuten im Museum von Kairo, Nr. 1-1294, Teil 4: Text und Tafeln zu Nr. 951-1294 (Catalogue général des antiquités égyptiennes du Musée du Caire), Berlin 1934.

Bosticco, S., Museo Archeologico di Firenze: le stele egiziane dall'Antico al Nuovo Regno, Firenze 1959.

Brunner, H., Die südlichen Räume des Tempels von Luxor (ArchVer 18), Berlin-Mainz 1977.

Brunner, H., dans W. Helck et E. Otto (éd.), $L \ddot{A} \mathrm{I}$, 1975, col. 805-11, s.v. « Bildliche Ausdrücke und Übertragungen $»$.

Bryan, B.M., " Administration in the Reign of Thutmose III », dans E.H. Cline et D.B. O’Connor (éd.), Thutmose III. A New Biography, Ann Arbor 2006, p. 69-122.

Buck, A. de, « The Building Inscription of the Berlin Leather Roll », dans A.M. Blackman, E. Otto, J. Vandier et A. de Buck (éd.), StudAeg I (AnOr 17), Roma 1938, p. 48-57.

Budge, E.A.W., Facsimiles on Egyptian Hieratic Papyri in the British Museum with Descriptions, Summaries of Contents etc., London 1923.

Bülow-Jacobsen, A., « Writing Materials in the Ancient World », dans R.S. Bagnall (éd.), The Oxford Handbook of Papyrology, Oxford-New York 2009, p. 3-29.

Blumenthal, E., A. Burkhardt, I. Müller et W.F. Reineke, Urkunden der 18. Dynastie. Übersetzung zu den Heften 5-16 [IV,315-1226] (Urk. IV), Berlin 1984.

Caminos, R.A., " Some Comments on the Reuse of
Papyrus », dans M.L. Bierbrier (éd.), Papyrus. Structure and Usage (BMOP 60), London 1986, p. 43-61.

Caminos, R.A., Late-Egyptian Miscellanies (BEStud 1), London 1954.

Caminos, R.A. et T.G.H. James, Gebel es-Silsilah, I. The Shrines (ASE 31), London 1963.

Cardin, Chr. et J.-Cl. Goyon (dir.), Trésors d'Égypte. La " cachette " de Karnak (1904-2004). Exposition en hommage à Georges Legrain à l'occasion du IXe Congrès International des égyptologues. Musée Dauphinois, 4 septembre 2004 - 5 janvier 2005 (catalogue de l'exposition, musée Dauphinois, Grenoble), Grenoble 2004.

Cenival, J.L. de, « Sur la forme $s \underline{d m} . f$ à redoublement ou $m r r . f », R d E 24$ (1972), p. 40-45.

Černý, J., A Community of Workmen at Thebes in the Ramesside Period (BiEtud 50), Le Caire $2001^{2}$.

Černý, J., « Troisième série de questions adressées aux oracles », BIFAO 72 (1972), p. 49-69.

Černý, J., « Egyptian Oracles », dans R.A. Parker (éd.), A Saite Oracle Papyrus from Thebes in the Brooklyn Museum (Papyrus Brooklyn 47.218.3) (BEStud 4), Providence (RI) 1962, p. 35-48.

Černý, J., Papers and Books in Ancient Egypt. An Inaugural Lecture Delivered at University College, London, 29 May 1947, London 1947.

Černý, J., « Nouvelle série de questions adressées aux oracles », BIFAO 41 (1942), p. 13-24.

Černý, J., « The Gender of Tens and Hundreds in Late Egyptian », JEA 23 (1937), p. 57-59.

Černý, J., « Questions adressées aux oracles », BIFAO 35 (1935), p. 41-58.

Černý, J., « Une expression désignant la réponse négative d'un oracle », BIFAO 30 (1931), p. 491-96.

Černý, J., Notebook, MSS 17.151.

Champollion, J.-Fr., Lettres à M. le duc de Blacas d'Aulps, premier gentilhomme de la chambre, pair de France, etc. relatives au musée royal égyptien de Turin, II. Suite des monuments historiques, Paris 1826.

Chantrain, G. et J. Winand, « L'adverbe gr en néoégyptien », RdE 63 (2012), p. 43-66.

Coche-Zivie, Chr., « Les colonnes du “ Temple de l'Est” à Tanis. Épithètes royales et noms divins », BIFAO 74 (1974), p. 93-121.

Collombert, Ph., « Khâemouaset, grand prêtre de Ptah et "prince archéologue" », dans A. Charron et Chr. Barbotin (dir.), Savoir et pouvoir à l'époque de Ramsès II. Khâemouaset le prince archéologue (catalogue de l'exposition, musée de l'Arles antique, Arles) Arles 2016, p. 42-43.

Coyette, A., « La naissance merveilleuse d'Hatshepsout dans les reliefs de Deir el-Bahari », dans Chr. Cannuyer et C. Vialle (éd.), Les naissances merveilleuses en Orient. Jacques Vermeylen (1942-2014) in memoriam, (AOB 28), Bruxelles 2015, p. 87-112.

Cumming, B., Egyptian Historical Records of the Later Eighteenth Dynasty, II, Warminster 1984.

Curto, S., Storia del Museo Egizio di Torino, Torino 1976.

Daressy, G., Ostraca, (CGC nos 25001-25385), Le Caire 1901.

Davies, N de G., The Tomb of Rekh-mi-Rée at Thebes, I-II (PMMA 11), New York 1943. 
Davies, N. de G., The Tomb of Ken-Amūn at Thebes, I-II (PMMA 5), New York 1930.

Davies, N. de G., Two Ramesside Tombs at Thebes (PMMA 2), New York 1927.

Davies, N. de G., The Rock Tombs of el Amarna, VI (ASEg 18), London 1908.

Davies, N. de G. et M.F.L. Macadam, A Corpus of Inscribed Egyptian Funerary Cones, I. Plates, Oxford 1957.

Davies, V. et D. Laboury (éd.), The Oxford Handbook of Egyptian Epigraphy and Palaeography, Oxford 2019.

von Deines, H. et W. Westendorf 1961 (éd.), Wörterbuch der medizinischen Texte. Erste Hälfte (3-r) (Grundriss der Medizin der alten Ägypter VII/1), Berlin 1961.

Del Vesco, P. et F. Poole, « Deir el-Medina in the Egyptian Museum of Turin: An Overview, and the Way Forward ", dans A. Dorn et St. Polis (éd.), Outside the Box. Selected Papers from the Conference "Deir el-Medina and the Theban Necropolis in Contact". Liège, 27-29 October 2014 (AegLeod 11), Liège, 2018, p. 97-130.

Demarée, R.J., The Bankes Late Ramesside Papyri (British Museum Research Publication 155), London 2006.

Demarée, R.J., Ramesside Ostraca, London 2002.

Demichelis, S., « Papiri calendariali del Museo Egizio di Torino », VicOr 11 (1999), p. 103-12.

Derchain, Ph., « Les débuts de l'histoire. Rouleau de cuir Berlin 3029 », RdE 43 (1992), p. 35-47.

Donker van Heel, K. et B.J.J. Haring, Writing in a Workmen's Village. Scribal Practice in Ramesside Deir el-Medina (EgUit 16), Leiden 2003.

Dorman, P.F., « The Early Reign of Thutmose III: An Unorthodox Mantle of Coregency », dans E.H. Cline et D. O'Connor (éd.), Thutmose III. A New Biography, Ann Arbor 2006, p. 39-68.

Dorman, P.F., The Monuments of Senenmut. Problems in Historical Methodology, London-New York 1988.

Dorn, A., T.J. Gillen et St. Polis, « Deir el-Medina Studies: Current Situation and Future Perspectives », dans A. Dorn et St. Polis (éd.), Outside the Box. Selected Papers from the Conference "Deir el-Medina and the Theban Necropolis in Contact”. Liège, 27-29 October 2014 (AegLeod 11), Liège 2018, p. 7-16.

Depuydt, L., " The Standard Theory of the "Emphatic" Forms in Classical (Middle) Egyptian: A Historical Survey », OLP 14 (1983), p. 13-54.

Devaud, E., « Études de lexicographie égyptienne et copte », Kêmi 2 (1929), p. 3-18.

Dresbach, G., Zur Verwaltung in der 20. Dynastie. Das Wesirat (KSG 9), Wiesbaden 2012.

Dziobek, E., Denkmäler des Vezirs User-Amun (SAGA 18), Heidelberg 1998.

Dziobek, E., « Theban Tombs as a Source for Historical and Biographical Evaluation: The Case of UserAmun ", dans J. Assmann, E. Dziobek, H. Guksch et Fr. Kampp (éd.), Thebanische Beamtennekropolen. Neue Perspektiven archäologischen Forschung (SAGA 12), Heidelberg 1995, p. 129-40.

Dziobek, E., Gräber des Vezirs User-Amun: Theben Nr. 61 und 131 (ArchVer 84), Berlin - Mainz 1994.

Edwards, I.E.S., " The Bankes Papyri I and II », JEA 68 (1982), p. 126-33.

Edwards, I.E.S., Hieroglyphic Texts from Egyptian Stelae, etc. in the British Museum, VIII, London 1939.
Eichler, S.S., Die Verwaltung des « Hauses des Amun » in der 18. Dynastie (BSAK 7), Hamburg 2000.

Eichler, S.S., « Amtseinsetzung und Beförderung von Beamten in der 18. Dynastie », SAK 25 (1998), p. 47-69.

Enmarch, R. et V.M. Lepper (éd.), Ancient Egyptian Literature. Theory and Practice. Proceedings of the Conference held at All Souls College, Oxford, from 1-3 September 2006 (PBA 188), Oxford 2013.

Erman, A., « Die Ankunft des Nils », ZÄS 85 (1960), p. 35-42.

Erman, A., « Gebete eines ungerecht Verfolgten und andere Ostraka aus den Königsgräbern », ZÄS 38 (1900), p. 19-41.

Erman, A., Die Märchen des Papyrus Westcar II (MOS 6), Berlin 1890.

Erman, A. et H. Grapow, Wörterbuch der Ägyptische Sprache I-V, Berlin - Leipzig 1926-1963.

Erman, A. et H.O. Lange, Papyrus Lansing. Eine ägyptische Schulhandschrift der 20. Dynastie, Copenhagen 1925.

Eyre, Chr.J., « Is Egyptian Historical Literature "Historical” or 'Literary"? ", dans A. Loprieno (éd.), Ancient Egyptian Literature. History and Forms (Problëg 10), Leiden 1996, p. 415-33.

Fabretti, A., Fr. Rossi, et R.V. Lanzone, Regio Museo di Torino. Antichità Egizie (Catalogo generale dei musei di antichità e degli oggetti d'arte raccolti nelle gallerie e biblioteche del regno, Serie Prima Piemonte, 1), I, Torino 1882.

Faulkner, R.O., A Concise Dictionary of Middle Egyptian, Oxford 1962.

Faulkner, R.O., « The Installation of the Vizier », JEA 41 (1955), p. 18-29.

Faulkner, R.O., « The Bremner-Rhind Papyrus - I », JEA 22 (1936), p. 121-40.

Faulkner, R.O., The Papyrus Bremner-Rhind (British Museum No. 10188) (BiAeg 3), Brussels 1933.

Feucht, E., Das Kind im Alten Ägypten. Die Stellung des Kindes in Familie und Gesellschaft nach altägyptischen Texten und Darstellungen, Frankfurt - New York 1995.

Fischer Elfert, H.-W., « Representations of the Past in New Kingdom Literature », dans J. Tait (éd.), "Never Had the Like Occurred". Egypt's View of its Past, London 2003, p. 119-37.

Fischer-Elfert, H.-W., Die satirische Streitschrift des Papyrus Anastasi I. Übersetzung und Kommentar

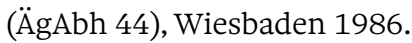

Friedrich, M., et C. Schwarke (éd.), One-Volume Libraries. Composite and Multiple-Text Manuscripts (Studies in Manuscript Cultures 9), Berlin 2016.

Galán, J.M., « The Ancient Egyptian Sed-Festival and the Exemption from Corvée ", JNES 59/4 (2000), p. 255-64.

Gale, N.H. et Z.A. Stos-Gale, « Ancient Egyptian Silver », JEA 67 (1981), p. 103-15.

Gardiner, A.H., Egyptian Grammar. Being an Introduction to the Study of Hieroglyphs, London $1957^{3}$.

Gardiner, A.H., Late-Egyptian Miscellanies (BiAeg 7), Brussels 1937.

Gardiner, A.H., Late-Egyptian Stories (BiAeg 1), Brussels 1932.

Gardiner, A.H., « The Autobiography of Rekhmerē », ZÄS 
60 (1925), p. 62-76.

Gardiner, A.H., Egyptian Hieratic Texts. Series I. Literary Texts of the New Kingdom. Part I. The Papyrus Anastasi I and the Papyrus Koller, Together with the Parallel Texts, Leipzig 1911.

Gauthier, H., Le livre des rois d'Égypte. Recueil des titres et protocoles royaux, noms propres de rois, reines, princes, princesses et parents de rois, suivi d'un index alphabétique II. De la XIII à la fin de la XVIII dynastie (MIFAO 18), Le Caire 1912.

Gnirs, A.M., « Zum Verhältnis von Literatur und Geschichte in der 18. Dynastie », dans S. Bickel (éd.), Vergangenheit und Zukunft. Studien zum historischen Bewusstsein in der Thutmosidenzeit (AegHelv 22), Basel 2013, p. 127-86.

Gnirs, A.M., « Geschichte und Literatur: wie 'historisch' sind ägyptische literarische Texte? ", dans G. Moers, K. Widmaier, A. Giewekemeyer, A. Lümers et R. Ernst (éd.), Dating Egyptian Literary Texts. "Dating Egyptian Literary Texts”, Göttingen, 9-12 June 2010 (LingAeg, Studia Monographica 11), Hamburg 2013, p. 367-403.

Gnirs, A.M., et A. Loprieno, « Krieg und Literatur », dans R. Gundlach et C. Vogel (éd.), Militärgeschichte des pharaonischen Ägypten. Altägypten und seine Nachbarkulturen im Spiegel der aktuellen Forschung, Paderborn 2008, p. 243-308.

Goedicke, H., « The Berlin Leather Roll (P Berlin 3029) », dans Festschrift zum 150jährigen Bestehen des Berliner Ägyptischen Museums (MÄS 8), Berlin - Munich 1974, p. 87-104.

Goelet Jr., O., « The Literary Environment of the Age of Ramesses III », dans E.H. Cline et D. O’Connor (éd.), Ramesses III. The Life and Times of Egypt's Last Hero, Ann Arbor 2012, p. 305-403.

Goelet Jr., O., « Writing Ramesside Hieratic: What the Late Egyptian Miscellanies Tell Us About Scribal Education ", dans S.H. D’Auria (éd.), Servant of Mut. Studies in Honor of Richard A. Fazzini (Probläg 28), Leiden 2008, p. 102-10.

Goody, J., The Interface Between the Written and the Oral, Cambridge 1987.

Graefe, E., Untersuchungen zur Wortfamilie bj3 : InauguralDissertation zur Erlangung des Doktorgrades der Philosophischen Fakultät der Universität zu Köln, Köln 1971.

Grandet, P., Les pharaons du Nouvel Empire. Une pensée stratégique (1550-1069 avant J.-C.), Paris 2008.

Grandet, P., Le papyrus Harris I (BM 9999), I-II (BiEtud 109/1-2), Le Caire 1994.

Grandet, P., « L’Égypte, comme institution, à l'époque ramesside », DiscEg 8 (1987), p. 72-92.

Grapow, H., Die bildlichen Ausdrücke des Ägyptischen, Leipzig 1924.

Grapow, H., « Zwei Fragmente einer Handschrift des Nilhymnus in Turin », ZÄS 52 (1914), p. 103-6.

Grimal, N., Les termes de la propagande royale égyptienne de la XIX dynastie à la conquête d'Alexandre (MAIBL n.s. 6), Paris 1986.

Grossman, E. et J. Cromwell, « Scribes, Repertoires, and Variation », dans J. Cromwell et E. Grossman (éd.), Scribal Repertoires in Egypt from the New Kingdom to the Early Islamic Period, Oxford 2018, p. 1-19.
Guglielmi, W., « Der Gebrauch rhetorischer Stilmittel in der ägyptischen Literatur », dans A. Loprieno (éd.), Ancient Egyptian Literature. History and Forms (Problëg 10), Leiden 1996, p. 465-97.

Guglielmi, W., dans W. Helck et E. Otto (éd.), LÄ VI, 1986, col. 22-41, s.v. « Stilmittel».

Guglielmi, W., dans W. Helck et E. Otto (éd.), LÄ VI, 1986, col. 986-89, s.v. « Vergleich ».

Hagen, Fr., « Archives in Ancient Egypt, 2500-1000 BCE », dans A. Bausi, Chr. Brockmann, M. Friedrich et S. Kienitz (éd.), Manuscripts and Archives. Comparative Views on Record-Keeping (Studies in Manuscript Cultures 11), Berlin - Boston 2018, p. 71-170.

Hagen, Fr., « An Eighteenth Dynasty Writing Board (Ashmolean 1948.91) and The Hymn to the Nile », JARCE 49 (2013), p. 73-91.

Hagen, Fr., An Ancient Egyptian Literary Text in Context. The Instruction of Ptahhotep (OLA 218), Leuven 2012.

Hagen, Fr., « Ostraca, Literature and Teaching at Deir elMedina », dans R. Mairs et A. Stevenson (éd.), Current Research in Egyptology 2005. Proceedings of the Sixth Annual Symposium, University of Cambridge, 6-8 January 2005, Oxford 2007, p. 38-51.

Hagen, Fr., « Literature, Transmission, and the Late Egyptian Miscellanies », dans R.J. Dann (éd.), Current Research in Egyptology 2004. Proceedings of the Fifth Annual Symposium Which Took Place at the University of Durham January 2004, Oxford 2006, p. 84-99.

Hannig, R., Ägyptisches Wörterbuch II. Mittleres Reich und Zweite Zwischenzeit (Hannig-Lexica 5, Kulturgeschichte der Antiken Welt 112), Mainz 2006.

Hannig, R., Ägyptisches Wörterbuch I. Altes Reich und Erste Zwischenzeit (Hannig-Lexica 4, Kulturgeschichte der Antiken Welt 98), Mainz 2003.

Haring, B.J.J., « Scribes and Scribal Activity at Deir elMedina », dans A. Dorn et T. Hofmann (éd.), Living and Writing in Deir el-Medine. Socio-Historical Embodiment of Deir el-Medine Texts (AegHelv 19), Basel 2006, p. 107-12.

Haring, B.J.J., « From Oral Practice to Written Record in Ramesside Deir el-Medina ", JESHO 46 (2003), p. 249-72.

Harris, J.R., Lexicographical Studies in Ancient Egyptian Minerals, Berlin 1961.

Helck, W., Urkunden der 18. Dynastie (Urkunden des ägyptischen Altertums IV, fasc. 17-22), Berlin $1984^{2}$.

Helck, W., Historisch-biographische Texte der 2. Zwischenzeit und neue Texte der 18. Dynastie (KÄT 6/1), Wiesbaden 1975.

Helck, W., Der Text des “Nilhymnus” (KÄT 4), Wiesbaden 1972.

Helck, W., Urkunden der 18. Dynastie. Übersetzung zu den Heften 17-22 [IV,1227-2179] (Urkunden des ägyptischen Altertums IV), Berlin 1961.

Helck, W., Zur Verwaltung des Mittleren und Neuen Reichs (Problëg 3), Leiden 1958.

Helck, W., « Die Berufung des Vezirs Wsr », dans O. Firchow, (éd.), Ägyptologische Studien. Hermann Grapow zum 70. Geburtstag gewidmet, Berlin 1955, p. 107-17.

Hintze, Fr., « Die statistische Struktur des Wortschatzes ägyptischer Literaturwerke », ZÄS 102 (1975), 
p. 100-22.

Hoogendijk, Fr.A.J. et St. Van Gompel (éd.), The Materiality of Texts from Ancient Egypt. New Approaches to the Study of Textual Material from the Early Pharaonic to the Late Antique Period (P.L.Bat. 35), Leiden-Boston 2018.

Hornung, E., Der ägyptische Mythos von der Himmelskuh. Eine Ätiologie des Unvollkommenen (OBO 46), Freiburg - Göttingen $1991^{2}$.

Hornung, E., Die Grabkammer des Vezirs User (NAWG 5), Göttingen 1961.

Hsu, Sh-W., Bilder für den Pharao. Untersuchungen zu den bildlichen Ausdrücken des Ägyptischen in den Königsinschriften und anderen Textgattungen (Probläg 36), Leiden 2017.

Jäger, St., Altägyptische Beruftypologien (LingAeg, Studia Monographica 4), Hamburg 2004.

Janssen, J.J., « Literacy and Letters at Deir el-Medîna », dans R.J. Demarée et A. Egberts (éd.), Village Voices. Proceedings of the Symposium "Texts from Deir elMedîna and Their Interpretation", Leiden, May 31 - June 1, 1991 (Centre of Non-Western Studies Publications 13), Leiden 1992, p. 81-94.

Janssen, R.M. et J.J. Janssen, Growing Up in Ancient Egypt, London 1990.

Jasnow, R., A Late Period Hieratic Wisdom Text (P. Brooklyn 47.218.135) (SAOC 52), Chicago 1992.

Jonckheere, Fr., « La circoncision des anciens égyptiens », Centaurus 1/3 (1951), p. 212-34.

Jones, D., An Index of Ancient Egyptian Titles, Epithets and Phrases of the Old Kingdom, I-II (BAR-IS 866), London 2000.

Junge, Fr., Late Egyptian Grammar. An Introduction, Oxford 2001.

Kákosy, L., dans W. Helck et W. Westendorf (éd.), $L \ddot{A} I V$, 1982, col. 600-6, s.v. « Orakel ».

Kampp-Seyfried, F., Die thebanische Nekropole. Zum Wandel des Grabgedankens von der 18. bis zur 20. Dynastie I-II (Theben 13), Mayance 1996.

Klug, A., Königliche Stelen in der Zeit von Ahmose bis Amenophis III (MonAeg 8), Brussels 2002.

$\mathrm{KRI}=$ Kitchen, K.A., Ramesside Inscriptions, Historical and Bibliographical, I-VIII, Oxford 1969-1990.

KRITA = Kitchen, K.A. puis Davies, B.G., Ramesside Inscriptions, Translated and Annotated. Notes and Comments, I-IV, Oxford - Cambridge (MA) - Malden (MA) 1993-2014.

KRITA = Kitchen, K.A., Ramesside Inscriptions, Translated and Annotated. Translations, I-VII, Malden (MA) Oxford 1993-2014.

KRITANC = Kitchen, K.A., Ramesside Inscriptions. Translated and Annotated. Series B: Annotations, Oxford 1993-1999.

Kruchten, J.-M., Les annales des prêtres de Karnak (XXIXXIII ${ }^{\text {mes }}$ dynasties) et autres textes contemporains relatifs à l'initiation des prêtres d'Amon (OLA 32), Leuven 1989.

Kruchten, J.-M., Le grand texte oraculaire de Djéhoutymose. Intendant du domaine d'Amon sous le pontificat de Pinedjem II (MRE 5), Brussels 1986.

Krutzsch, M., " Einzelblatt und Rolle: zur Anatomie von Papyrushandschriften », dans Fr. Feder, G. Sperveslage et Fl. Steinborn (éd.), Ägypten begreifen. Erika
Endesfelder in Memoriam, London 2017, p. 213-22.

Krutzsch, M., « Reading Papyrus as Writing Material », BMSAES 23 (2016), p. 57-69.

Krutzsch, M., « Materialtechnische Beobachtungen während der Restaurierung », dans H.W. Fischer-Elfert (éd.), Magika Hieratika in Berlin, Hannover, Heidelberg und München, Berlin - München - Boston 2014, p. 1-74.

Krutzsch, M., « Falttechniken an Handschriften aus dem alten Ägypten », dans B. Backes, I. Munro et S. Stöhr (éd.), Totenbuch-Forschungen. Gesammelte Beiträge des 2. Internationalen Totenbuch-Symposiums, Bonn, 25. bis 29. September 2005 (SAT 11), Wiesbaden 2006, p. 167-95.

Graf, J. et M. Krutzsch (éd.), Ägypten lesbar machen. Die klassische Konservierung/Restaurierung von Papyri und neuere Verfahren. Gesammelte Beiträge des 1. Internationalen Workshops der Papyrusrestauratoren, Leipzig 7.-9. September 2006 (APF 24), Berlin-Boston 2008.

Kuentz, Ch., « La "stèle du mariage" de Ramsès II », ASAE 25 (1925), p. 181-238.

Laboury, D., La statuaire de Thoutmosis III. Essai d'interprétation d'un portrait royal dans son contexte historique (AegLeod 5), Liège 1998.

Leach, Br. et J. Tait, « Papyrus », dans P.T. Nicholson et I. Shaw (éd.), Ancient Egyptian Materials and Technology, Cambridge 2000, p. 227-53.

Leclant, J., « Les inscriptions “éthiopiennes” sur la porte du IVe pylône du grand temple d'Amon à Karnak ", $R d E 8$ (1951), p. 104-13.

Lefebvre, G., Inscriptions concernant les grands prêtres d'Amon Romê-Rö̈ et Amenhotep (WZKM 41), Paris 1929.

Lepper, V.M., « Language and “Text” », LingAeg 14 (2006), p. $375-88$.

Lesko, L.H. et B.S. Lesko, A Dictionary of Late Egyptian, I-II, Providence 2002-2004 (2e éd.).

Lichtheim, M., Ancient Egyptian Literature. A Book of Readings, I-III, Berkeley (CA) - London 1973-1980.

Lloyd, A.B., « The Great Inscription of Khnumhotpe II at Beni Hasan », dans A.B. Lloyd (éd.), Studies in Pharaonic Religion and Society in Honour of J. Gwyn Griffith (EES Occasional Publication 8), London 1992, p. 21-36.

Loprieno, A., " The "King's Novel” ", dans A. Loprieno (éd.), Ancient Egyptian Literature. History and Forms (Probläg 10), Leiden 1996, p. 277-95.

Loprieno, A., « Linguistic Variety and Egyptian Literature », dans A. Loprieno, A. (éd.), Ancient Egyptian Literature. History and Forms (Problëg 10), Leiden 1996, p. 515-29.

Lorton, D., " Sections 7 to 11 of "The Duties of the Vizier" ", dans B.M. Bryan et D. Lorton (éd.), Essays in Egyptology in Honor of Hans Goedicke, San Antonio 1994, p. 147-55.

Lucas, A., Ancient Egyptian Materials and Industries, London $1962^{4}$.

Lucas, A., " The Chemistry of the Tomb », dans H. Carter (éd.), The Tomb of Tut-ankh-Amen. Discovered by the late Earl of Carnarvon and Howard Carter, III, London 1933, p. 170-83. 
Lucas, A., « Silver in Ancient Times », JEA 14 (1928), p. 313-19.

Lucas, A., " The Chemistry of the Tomb », dans H. Carter (éd.), The Tomb of Tut-ankh-Amen. Discovered by the late Earl of Carnarvon and Howard Carter, II, London 1927, p. 162-88.

Maltomini, F., « Use and Reuse of Papyrus Rolls and Scraps: Some Bibliological Matters », dans T. Derda, A. Lajtar, J. Urbanik (éd.), Proceedings of the $27^{\text {th }}$ International Congress of Papyrology. Warsaw, 29 July-3 August 2013 II, Warsaw 2016, p. 1097-112.

Manassa, C., Imagining the Past. Historical Fiction in New Kingdom Egypt, Oxford 2013.

Manassa, C., « Defining Historical Fiction in New Kingdom Egypt », dans S.C. Melville et A.L. Slotsky (éd.), Opening the Tablet Box. Near Eastern Studies in Honor of Benjamin R. Foster, Leiden 2010, p. 245-69.

Maruéjol, Fl., Thoutmosis III et la corégence avec Hatchepsout, Paris $2014^{2}$.

Maspero, G., Hymne au Nil (BiEtud 5), Le Caire 1912.

Mathieu, B., « La “Satire des Métiers”. Dossier bibliographique », Grafma Newsletter 2 (1998), p. 37-40.

Mathieu, B., « La "Satire des Métiers” (2) », Grafma Newsletter 3-4 (2001), p. 65-73.

Mathieu, B., «Réflexions sur le "Fragment Daressy" et ses hommes illustres », dans Chr. Zivie-Coche et I. Guermeur (éd.), "Parcourir l'éternité". Hommages à Jean Yoyotte 2 (BEHE 156), Paris 2012, p. 819-52.

Maystre, Ch., « Le Livre de la Vache du Ciel dans les tombeaux de la Vallée des Rois », BIFAO 40 (1941), p. 53-115.

McDowell, A., « Awareness of the Past in Deir el-Medîna », dans R.J. Demarée et A. Egberts (éd.), Village Voices. Proceedings of the Symposium "Texts from Deir elMedina and their Interpretation", Leiden, May 31 - June 1, 1991 (Centre of Non-Western Studies 13), Leiden 1992, p. 95-109.

Meeks, D., Mythes et légendes du Delta d'après le papyrus Brooklyn 47.218.84 (MIFAO 125), Le Caire 2006.

Meeks, D., review of Helck, W., Der Text des “Nilhymnus" (KÄT 4), Wiesbaden 1972, in: BiOr 32/1 (1975), p. 18-25.

Megahed, M. et H. Vymazalová, « Ancient Egyptian Royal Circumcision from the Pyramid Complex of Djedkare », Anthropologia 49/2 (2011), p. 155-64.

Megally, M., Considérations sur les variations et la transformation des formes hiératiques du papyrus E. 3226 du Louvre (BiEtud 49), Le Caire 1971.

Ménei, E., « Le papyrus comme support d'écriture », dans Laroque, $\mathrm{Cl}$. (dir.), Autour des papiers asiatiques. Actes des colloques D'est en Ouest. Relations bilatérales autour du papier en l'Extrême-Orient et l'Occident (organisé le 10 octobre 2014) et Papiers et protopapiers. Les supports de l'écrit ou de la peinture (organisé le 30 octobre 2015), Paris, site de l'HiCSA, mis en ligne en février 2017 (https://hicsa.univ-paris1.fr/documents/ pdf/PublicationsLigne/Actes\%20Laroque\%20 2017/08_Menei.pdf, dernière consultation le 17/12/2018), p. 138-60.

Ménei, E., « Remarques sur la fabrication des rouleaux de papyrus : précisions sur la formation et l'assemblage des feuillets », RdE 44 (1993), p. 185-88.
Moers, G., « Fiktionalität und Intertextualität als Parameter ägyptologischer Literaturwissenschaft. Perspektiven und Grenzender Anwendung zeitgenössischer Literaturtheorie », dans J. Assmann et E. Blumenthal (éd.), Literatur und Politik im pharaonischen und ptolemäischen Ägypten. Vorträge der Tagung zum Gedenken an Georges Posener, 5.-10. September 1996 in Leipzig (BEC 127), Le Caire 1999, p. 37-52.

Möller, G., « Zur Datierung literarischer Handschriften aus der ersten Hälfte des Neuen Reichs », ZÄS 56 (1920), p. 34-43.

Möller, G., Hieratische Paläographie. Die ägyptische Buchschrift in ihrer Entwicklung von der fünften Dynastie bis zur römischen Kaiserzeit, I-III, Leipzig 1909-1912.

Murnane, W.J., Texts from the Amarna Period in Egypt (Writings from the Ancient World 5), Atlanta 1995.

Naville, E., The Temple of Deir el Bahari I-VI (MEEF 13, 14, 16, 19, 27, 29), London 1895.

Neveu, Fr., La langue des Ramsès. Grammaire du Néoégyptien, Paris 1996.

Newberry, P.E. et F. Ll. Griffith, Beni Hassan I (ASEg 1), London 1893.

Obsomer, Cl., Sésostris I Ir. Étude chronologique et historique du règne (Connaissance de l'Egypte Ancienne 5), Bruxelles 1995.

Obsomer, Cl., Ramsès II: [Abou Simbel, Louxor, Néfertary, Qadech]. Les grands pharaons, Paris 2012.

Ockinga, B., « The burden of Kha'kheperrē'sonbu », JEA 69 (1983), p. 88-95.

Ogden, J., « Metals », dans P.T. Nicholson et I. Shaw (éd.), Ancient Egyptian Materials and Technology, Cambridge 2000, p. 148-76.

Oréal, E., Les particules en égyptien ancien. De l'ancien égyptien à l'égyptien classique (BiEtud 152), Le Caire 2011, p. 437-86.

Osing, J., dans W. Helck et E. Otto (éd.), LÄ II, 1977, col. 618-24, s.v. « Gleichnis ».

Parkinson, R. et St. Quirke, Papyrus, London 1995.

Petrie, W.M.Fl., A History of Egypt, II. During the XVII th and XVIII ${ }^{\text {th }}$ Dynasties, London $1899^{3}$.

Pinarello, M.S., Archaeological Discussion of Writing Practice. Deconstruction of the Ancient Egyptian Scribe, London 2015.

Piquette, K.E. et R.D. Whitehouse (éd.), Writing as Material Practice. Substance, Surface and Medium, London 2013.

Pleyte, W. et Fr. Rossi, Les papyrus hiératiques de Turin, I-II, Leiden 1869-1876.

PN = Ranke, H., Die ägyptischen Personennamen, I-III, Glückstadt - Hambourg 1935-1977.

Polis, St., « Linguistic Variation in Ancient Egyptian: An Introduction to the State of the Art (with Special Attention to the Community of Deir el-Medina) », dans J. Cromwell et E. Grossman (éd.), Scribal Repertoires in Egypt from the New Kingdom to the Early Islamic Period, Oxford 2018, p. 60-88.

Polotsky, H.J., « Les transpositions du verbe en égyptien classique », IOS 6 (1976), p. 1-50.

Polz, D., Der Beginn des Neuen Reiches. Zur Vorgeschichte einer Zeitenwende (SDAIK 31), Berlin-New York 2007. Porter, B. et R.L.B. Moss, Topographical Bibliography 
of Ancient Egyptian Hieroglyphic Texts, Reliefs and Paintings, I-VI, Oxford 1960-1974².

Quack, J.Fr., « Lobpreis der Gottheit und Hoffnung auf Beistand im spätramessidischen Ägypten: eine Neubearbeitung der sogenannten "Gebete eines ungerecht Verfolgten », dans A. Grund, A. Krüger et Fl. Lippke (éd.), Ich will dir danken unter den Völkern. Studien zur israelitischen und altorientalischen Gebetsliteratur. Festschrift für Bernd Janowski zum 70. Geburtstag, Gütersloh 2013, p. 557-93.

Quack, J.Fr., " Hymnus an den chthonischen Osiris », dans B. Janowski et D. Schwemer (éd.), Hymnen, Klagelieder und Gebete (Texte aus der Umwelt des Alten Testaments, Neue Folge 7), Gütersloh 2013, p. 145-272.

Quack, J.Fr., « Irrungen, Wirrungen? Forscherische Ansätze zur Datierung der älteren ägyptischen Literatur », dans G. Moers, K. Widmaier, A. Giewekemeyer, A. Lümers et R. Ernst (éd.), Dating Egyptian Literary Texts. "Dating Egyptian Literary Texts”, Göttingen, 9-12 June 2010 (LingAeg-Studia Monographica 11), Hamburg 2013, p. 405-69. Quack, J.Fr., « Zur Beschneidung im alten Ägypten », dans A. Berlejung, J. Dietrich et J.Fr. Quack (éd.), Menschenbilder und Körperkonzepte im Alten Israel, in Ägypten und im Alten Orient (Orientalische Religionen in der Antike 9), Tübingen 2012, p. 561-651.

Quack, J.F., « Die Lehre des Papyrus Brooklyn 47.218.135 », dans F. Hoffmann et J.F. Quack (éd.), Anthologie der demotischen Literatur (EQA 4), Münster 2007, p. 230-38.

Quack, J.F., « Die Initiation zum Schreiberberuf im Alten Ägypten », SAK 36, 2007, p. 249-95.

Quack, J.F., « Königsweihe, Priester, Isisweihe », dans J. Assmann et M. Bommas (éd.), Ägyptische Mysterien?, München, 2002, p. 95-108.

Quack, J.F., « Ein neuer ägyptischer Weisheitstext », WdO 24 (1993), p. 5-19.

Quirke, St., Titles and Bureaux of Egypt 1850-1700 BC (GHP Egyptology 1), London 2004.

Quirke, St., « Archive », dans A. Loprieno (éd.), Ancient Egyptian Literature. History and Forms (Problëg 10), Leiden 1996, p. 379-401.

Raedler, Chr., « Die Wesire Ramses’ II.: Netzwerke der Macht », dans R. Gundlach et A. Klug (éd.), Das ägyptische Königtum im Spannungsfeld zwischen Innenund Aussenpolitik im 2. Jahrtausend v. Chr. (Königtum, Staat und Gesellschaft früher Hochkulturen, Band 1), Wiesbaden 2004, p. 277-416.

Ragazzoli, Chl., La grotte des scribes à Deir el-Bahari. La tombe MMA 504 et ses graffiti (MIFAO 135), Le Caire 2017.

Redford, D.B., The Wars in Syria and Palestine of Thutmose III (Culture and History of the Ancient Near Eeast 16), Leiden 2003.

Ritner, R.K. et N. Moeller, « The Ahmose “Tempest Stela”, Thera and Comparative Chronology », JNES 73/1 (2014), p. 1-19.

Roccati, A., " Scavi nel Museo Egizio di Torino (VII. TRA I PAPIRI TORINESI) », Archaeogate (2008), p. 1-9 (article publié en ligne le 16/06/2008, http://www. archaeogate.org/egittologia/article.php?id=283 [URL non valide à présent]).
Roccati, A., « Papiri », dans A.M. Donadoni Roveri (éd.), Passato e futuro del Museo Egizio di Torino. Dal Museo al Museo. Archivi di archeologia, Torino 1989, p. 120-28.

Roccati, A., « Conservatività dell'egiziano », dans F.A. Pennacchietti et A. Roccati (éd.), Atti della terza giornata di studi camito-semitici e indoeuropei, Roma 1984, p. 107-15.

Roccati, A., « Les papyrus de Turin », BSFE 99 (1984), p. 9-27.

Roccati, A., « Scavi nel Museo di Torino VII. Tra i papiri Torinesi », OrAnt 14/3, (1975), p. 243-53.

Ryholt, K., « Scribal Habits at the Tebtunis Temple Library: On Materiality, Formal Features, and Palaeography », dans J. Cromwell et E. Grossman (éd.), Scribal Repertoires in Egypt from the New Kingdom to the Early Islamic period, Oxford 2018, p. 153-83.

Sandman, M., Texts from the Time of Akhenaten, (BiAeg 8), Brussels 1938.

Schmidt, Th., « Les palimpsestes littéraires grecs sur papyrus », dans V. Somers et B. Coulie (éd.), Palimpsestes et édition de textes. Les textes littéraires. Actes du colloque tenu à Louvain-la-Neuve, 5-6 septembre 2003 (PIOL 56), Leuven 2009, p. 83-99.

Schmidt, Th., « Greek Palimpsest Papyri: Some Open Questions », dans J. Frösén, T. Purola et E. Salmenkivi (éd.), Proceedings of the $24^{\text {th }}$ International Congress of Papyrology. Helsinki, 1-7 August, 2004, II, Helsinki 2007, p. 979-90.

Schneider, Th., Asiatische Personennamen in ägyptischen Quellen des Neuen Reiches (OBO 114), FreiburgGöttingen 1992.

Schwarz, St., « Zur Symbolik weisser und silberner Sandalen », ZÄS 123 (1996), p. 69-84.

Schweitzer, S.D., « Dating Egyptian Literary Texts: Lexical Approaches », dans G. Moers, K. Widmaier, A. Giewekemeyer, A. Lümers et R. Ernst (éd.), Dating Egyptian Literary Texts, Göttingen, 9-12 June 2010 (LingAeg-Studia Monographica 11), Hamburg 2013, p. 177-90.

Schiff Giorgini, M., Soleb V. Le temple. Bas-reliefs et inscriptions (BiGen 19), Le Caire 1998.

Sethe, K., Urkunden der 18. Dynastie. HistorischBiographische Urkunden (Urk. IV/1-4, fasc. 1-16), Berlin 1961².

Sethe, K., Historisch-biographische Urkunden des Mittleren Reiches [VII,1-66] (Urk. VII/1), Leipzig 1935.

Sethe, K., Urkunden der 18. Dynastie. [Übersetzung zu IV,1313] (Urk. IV), Leipzig 1914.

Shirley, J.J., " The Power of the Elite: The Officials of Hatshepsut's Regency and Coregency », dans J.M. Galán, B.M. Bryan et P.F. Dorman (éd.), Creativity and Innovation in the Reign of Hatshepsut. Papers from the Theban Workshop 2010 (SAOC 69), Chicago 2014, p. 173-245.

Silverman, D.P., « Verbal Nominal Clauses in Middle Egyptian », dans P. Posener-Kriéger (éd.), Mélanges Gamal eddin Mokhtar, II, Le Caire 1985, p. 269-85.

Smith, H.S., The Fortress of Buhen. The Inscriptions (ExcMem 48), London 1976.

Snape, S., « Khaemwese and the Present Past: History and the Individual in Ramesside Egypt », dans S. Snape et M. Collier (éd.), Ramesside Studies in Honour of K.A. 
Kitchen, Bolton 2011, p. 465-73.

Spalinger, A.J., The Transformation of an Ancient Egyptian Narrative. P. Sallier III and the Battle of Kadesh (GOF 40), Wiesbaden 2002.

Spigelman, M., « The Circumcision Scene in the Tomb of Ankhmahor: The First Record of Emergency Surgery? », BACE 8 (1997), p. 91-100.

Stauder, A., «Égyptien », Annuaire de l'EPHE, Section des sciences historiques et philologiques 147 (2016), http:// ashp.revues.org/1799.

Stauder, A., « La Königsnovelle : indices génériques, significations, écarts intertextuels », dans S. Vuilleumier et P. Meyrat (éd.), Mélanges pour une collègue distinguée, § 8 (à paraître dans la collection BiEtud, Le Caire).

Stauder, A., Linguistic Dating of Middle Egyptian Literary Texts. "Dating Egyptian Literary Texts", Göttingen, 9-12 June 2010 (LingAeg- Studia Monographica 12), Hamburg 2013.

Stauder, A., « The Earlier Egyptian "Emphatic” Construction: An Alternative Analysis ", dans J.P. Allen, M.A. Collier et A. Stauder (éd.), Coping with Obscurity. The Brown Workshop on Earlier Egyptian Grammar, Atlanta 2016, p. 169-99.

Stauder, A., The Earlier Egyptian Passive. Voice and Perspective (LingAeg-Studia Monographica 14), Hamburg 2014.

Tallet, P., « La fin des Devoirs du vizir », dans E. Warmenbol et V. Angenot (éd.), Thèbes aux 101 portes. Mélanges à la mémoire de Roland Tefnin (MonAeg 12, Série IMAGO n 3), Bruxelles 2010, p. 153-63.

Tallet, P., « Un nouveau témoin des “Devoirs du vizir” dans la tombe d'Aménémopé (Thèbes, TT 29) », ChronEg LXXX (159) (2005), p. 66-75.

The Epigraphic Survey, Medinet Habu VIII. The Eastern High Gate, with Translations of Texts (OIP 94), Chicago 1970.

Théodoridès, A., « À propos de la loi dans l'Égypte pharaonique », RIDA 14 (1967), p. 107-52.

Töpfer, S., « The Turin Papyrus Online Platform (TPOP): An Introduction », Rivista del Museo Egizio 2 (2018), p. 1-11 (DOI : 10.29353/rime.2018.1916).

Turner, E.G., « The Terms Recto and Verso: The Anatomy of the Papyrus Roll », dans J. Bingen et G. Nachtergael (éd.), Actes du XVe Congrès International de Papyrologie I (PapBrux 16), Bruxelles 1978.

Valbelle, D., "Les ouvriers de la tombe". Deir el-Médineh à l'époque Ramesside (BiEtud 96), Le Caire 1985.

Van der Plas, D., « Dating the Hymn to Hapi. An Update of the Late Date », dans G. Moers, K. Widmaier, A. Giewekemeyer, A. Lümers et R. Ernst (éd.), Dating Egyptian Literary Texts. Göttingen, 9-12 June 2010 (LingAeg-Studia Monographica 11), Hamburg 2013, p. 471-82.

Van der Plas, D., L’hymne à la crue du Nil, I-II (EgUit 4), Leiden 1986.

Van der Plas, D., « On Criteria for the Dating of Egyptian Texts », GöttMisz 73 (1984), p. 49-56.

Vandersleyen, $\mathrm{Cl}$. , « Une tempête sous le règne d'Amosis », RdE 19 (1967), p. 123-59.

Vandier, J., Le papyrus Jumilhac, Paris 1961.

Vernus, P., « The Circulation of "Literary” Texts in the Deir
el-Medina Community: Two Opposite Cases ", dans A. Dorn et St. Polis (éd.), Outside the Box. Selected Papers from the Conference "Deir el-Medina and the Theban Necropolis in Contact”. Liège, 27-29 October 2014 (AegLeod 11), Liège 2018, p. 477-92.

Vernus, P., Sagesses de l'Égypte pharaonique, Paris $2010^{2}$.

Vernus, P., Essai sur la conscience de l'histoire dans l'Égypte pharaonique (BEHE 332), Paris 1995.

Vernus, P., « Omina calendériques et comptabilité d'offrandes sur une tablette hiératique de la XVIII dynastie », RdE 33 (1981), p. 89-124.

Vernus, P., « Inscriptions de la Troisième Période Intermédiaire (IV) : le texte oraculaire réemployé dans le passage axial du IIIe pylône dans le temple de Karnak », CahKarn 6 (1973-1977), p. 215-33.

Ward, W.A., « Some Foreign Personal Names and LoanWords from the Deir el-Medineh Ostraca », dans Jr.A. Leonard et B.B. Williams (éd.), Essays in Ancient Civilization Presented to Helene J. Kantor (SAOC 47), Chicago 1989, p. 287-303.

Ward, W.A., Index of Egyptian Administrative and Religious Titles of the Middle Kingdom, Beyrouth 1982.

Weil, A., Die Veziere des Pharaonenreiches. Chronologisch Angeordnet von Arthur Weil, Strassburg 1908.

Wente, E.F., Letters from Ancient Egypt (Writings from the Ancient World 1), Atlanta 1990.

Westendorf, W., dans W. Helck, et E. Otto (éd.), L $2 \ddot{A}$ I, 1975, col. 727-29, s.v. « Beschneidung ».

Westendorf, W., « Das geminierende passive $s \underline{d m} . f(m r r . f)$ : imperfektisch oder emphatisch ? », Z̈̈S 84 (1959), p. 147-55.

Wiedemann, A., Ägyptische Geschichte 1. Von den ältesten Zeiten bis zum Tode Tutmes' III., Gotha 1884.

Willems, H., " A Note on Circumcision in Ancient Egypt », dans M.C. Flossmann-Schütze, M. Goecke-Bauer, Fr. Hoffmann, A. Hutterer, K. Schlüter, A. Schütze et M. Ullmann (éd.), Kleine Götter - Grosse Götter Festschrift für Dieter Kessler zum 65 (Tuna el-Gebel 4), Vaterstetten 2013, p. 553-58.

Wilson, J.A., « Egyptian Hymns and Prayers », dans J.B. Pritchard, (éd.), Ancient Near Eastern Texts Relating to the Old Testament, Princeton $1955^{2}$, p. 372-73.

Wimmer, St., Hieratische Paläographie der nichtliterarischen Ostraka der 19. und 20. Dynastie. Teil 1: Text. Teil 2: Tafeln (ÄAT 28), Wiesbaden 1995.

Winand, J., « Dialectal, Sociolectal and Idiolectal Variations in the Late Egyptian Texts from Deir elMedineh and the Theban Area », dans A. Dorn et St. Polis (éd.), Outside the Box. Selected Papers from the Conference "Deir el-Medina and the Theban Necropolis in Contact”. Liège, 27-29 October 2014 (AegLeod 11), Liège 2018, p. 493-524.

Winand, J., « Une nouvelle sagesse en hiératique de la Basse Époque (Papyrus Brooklyn Museum 47.218.135) », ChronEg 73 (145) (1998), p. 42-53.

Winand, J., Études de néo-égyptien 1. La morphologie verbale (AegLeod 2), Liège 1992.

Winand, J., « Champ sémantique et structure en égyptien ancien. Les verbes exprimant la vision », SAK 13 (1986), p. 293-314.

Wit, C. de, « La circoncision chez les anciens Égyptiens », ZÄS 99 (1972), p. 41-48. 
Yoyotte, J., « Un porche doré : la porte du IVe pylône au grand temple de Karnak », ChronEg 28 (55) (1953), p. 28-38.

Zonhoven, L., « Studies on the $s \underline{d} m . t=f$ Verb Form in

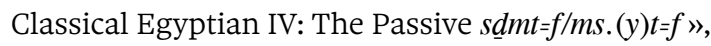
Z̈̈S 125 (1998), p. 78-92.
Sources en ligne

Griffith Institute, http://archive.griffith.ox.ac.uk. Projet Karnak, http://sith.huma-num.fr/karnak.

Thesaurus Linguae Aegyptiae (TLA), http://aaew.bbaw.de/ tla/.

Vocabulaire de l'Égyptien Ancien (VÉgA), http://vegavocabulaire-egyptien-ancien.fr/acceder-a-loutil/. Wortdiskussionen (par B. Lüscher et G. Lapp), https:// aegyptologie.philhist.unibas.ch/de/forschung/ werkzeuge/wortdiskussionen/.

;

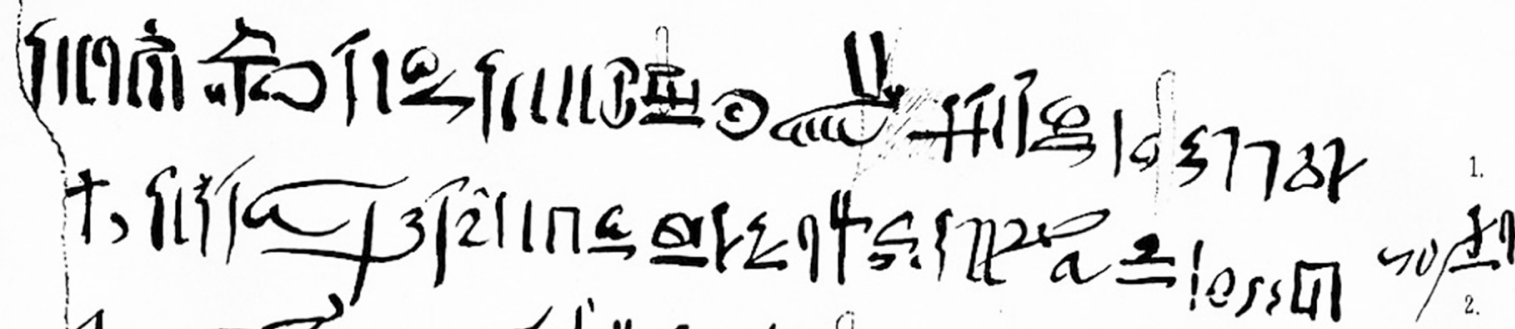
r

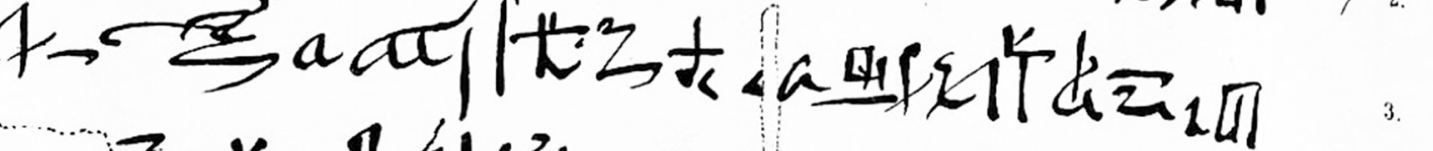

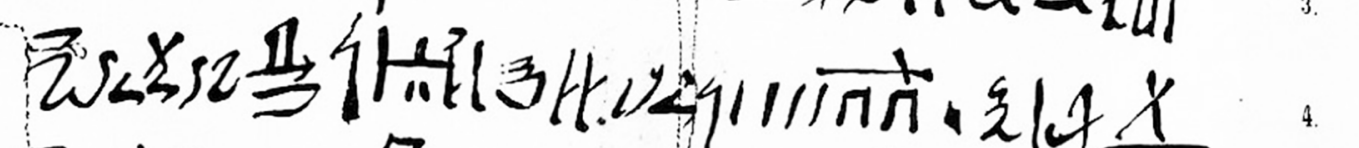

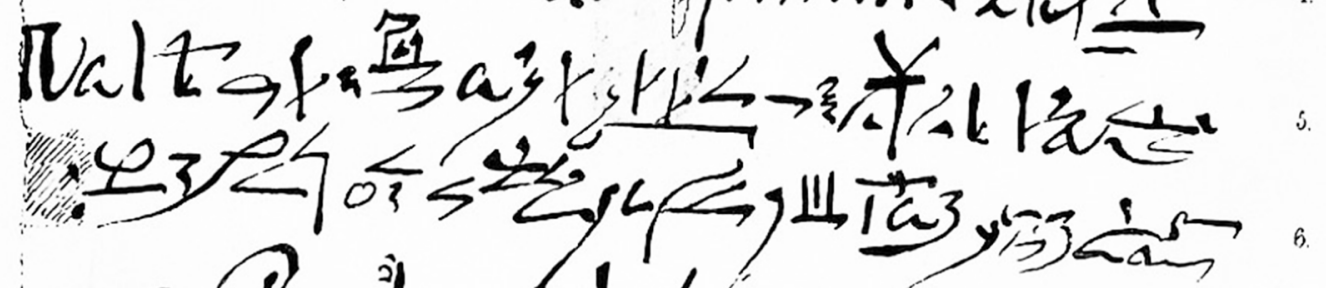

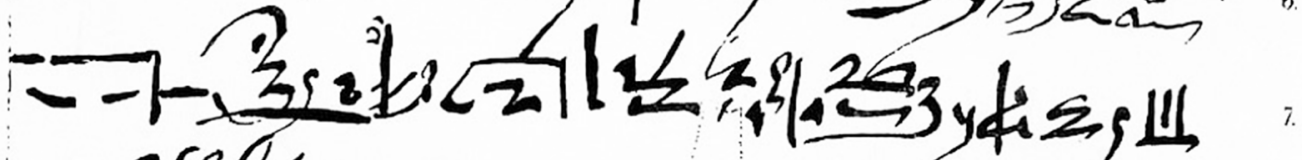

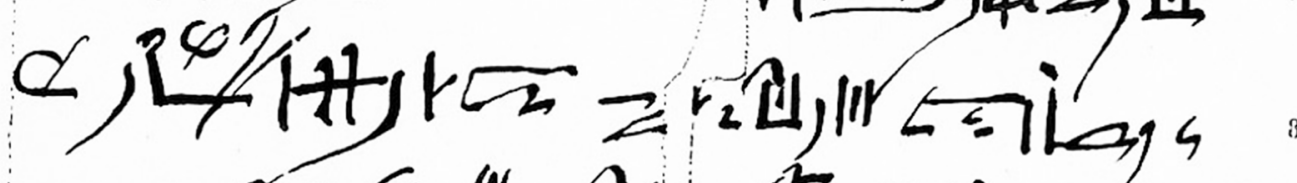

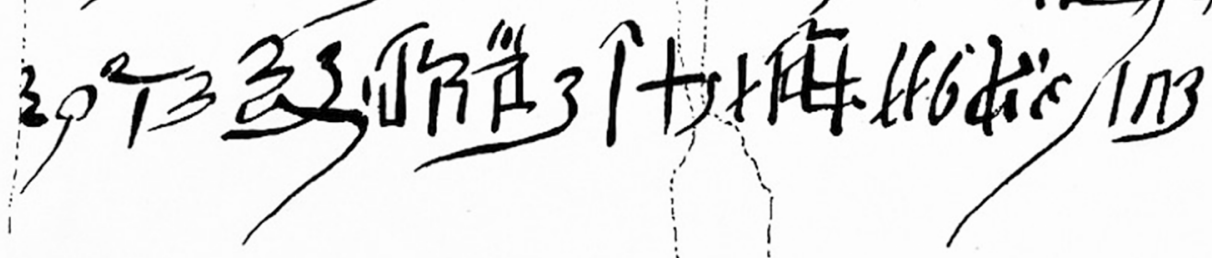

Thotmes III XVIII ieme dunastio 


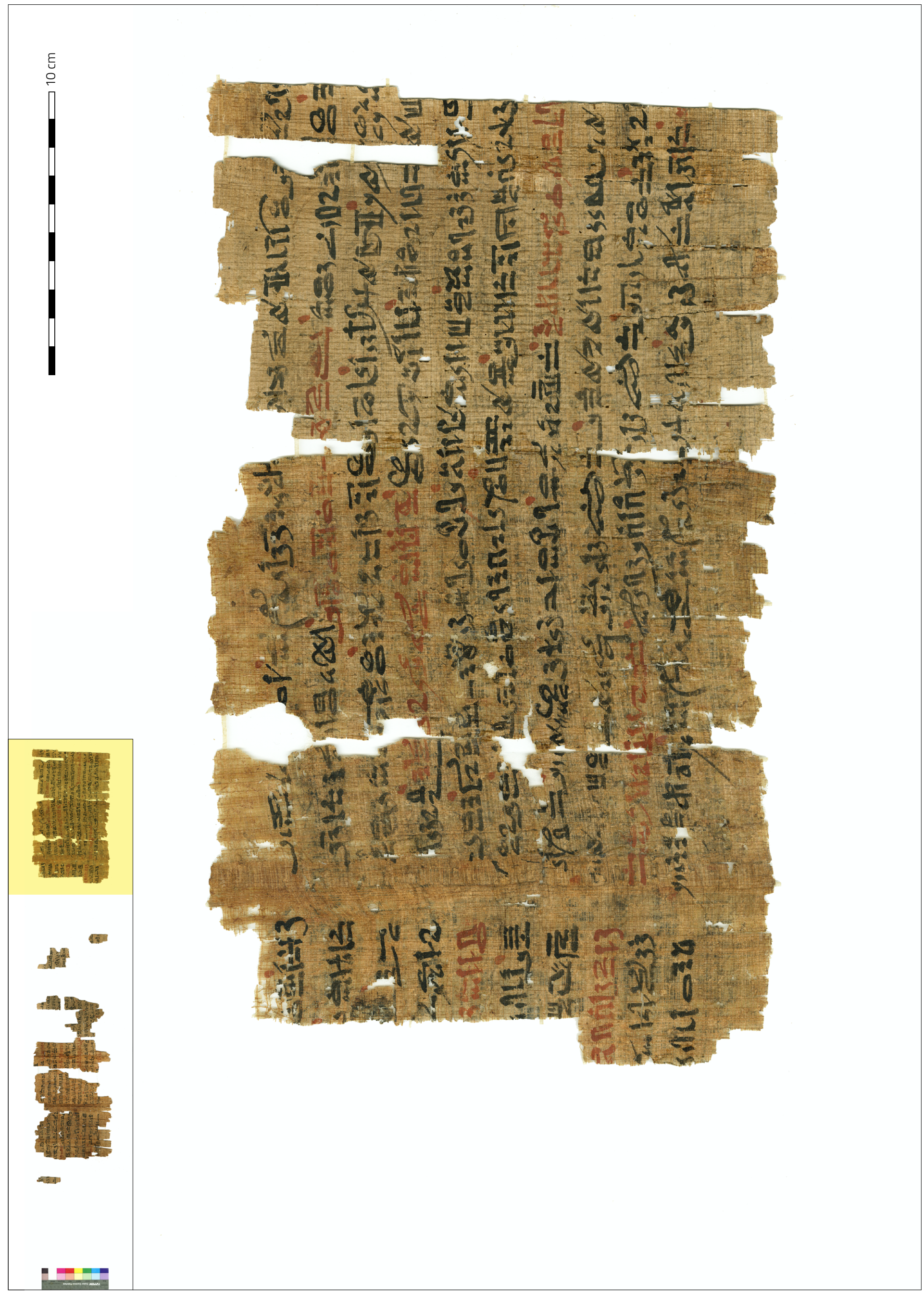


๒్
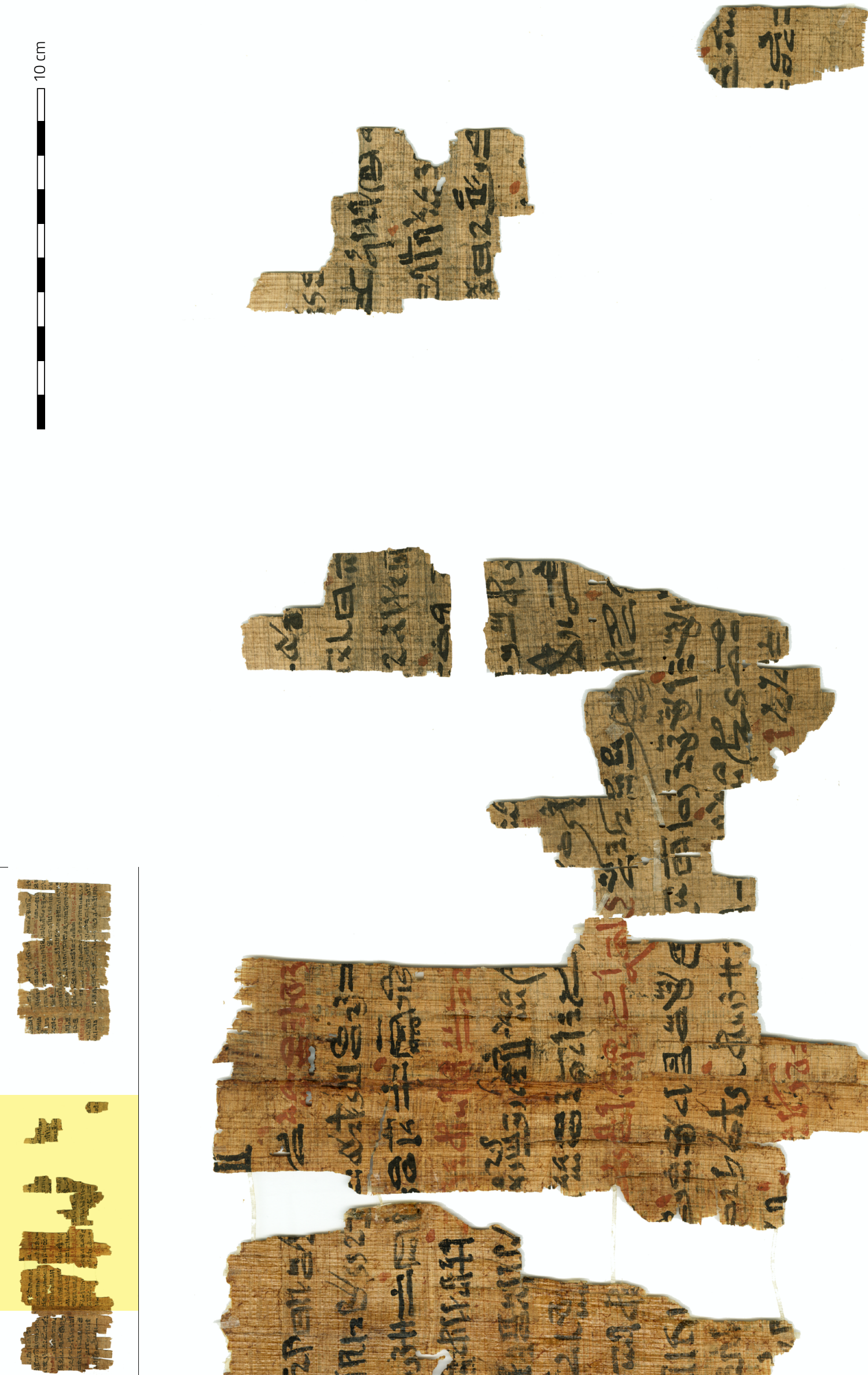

-
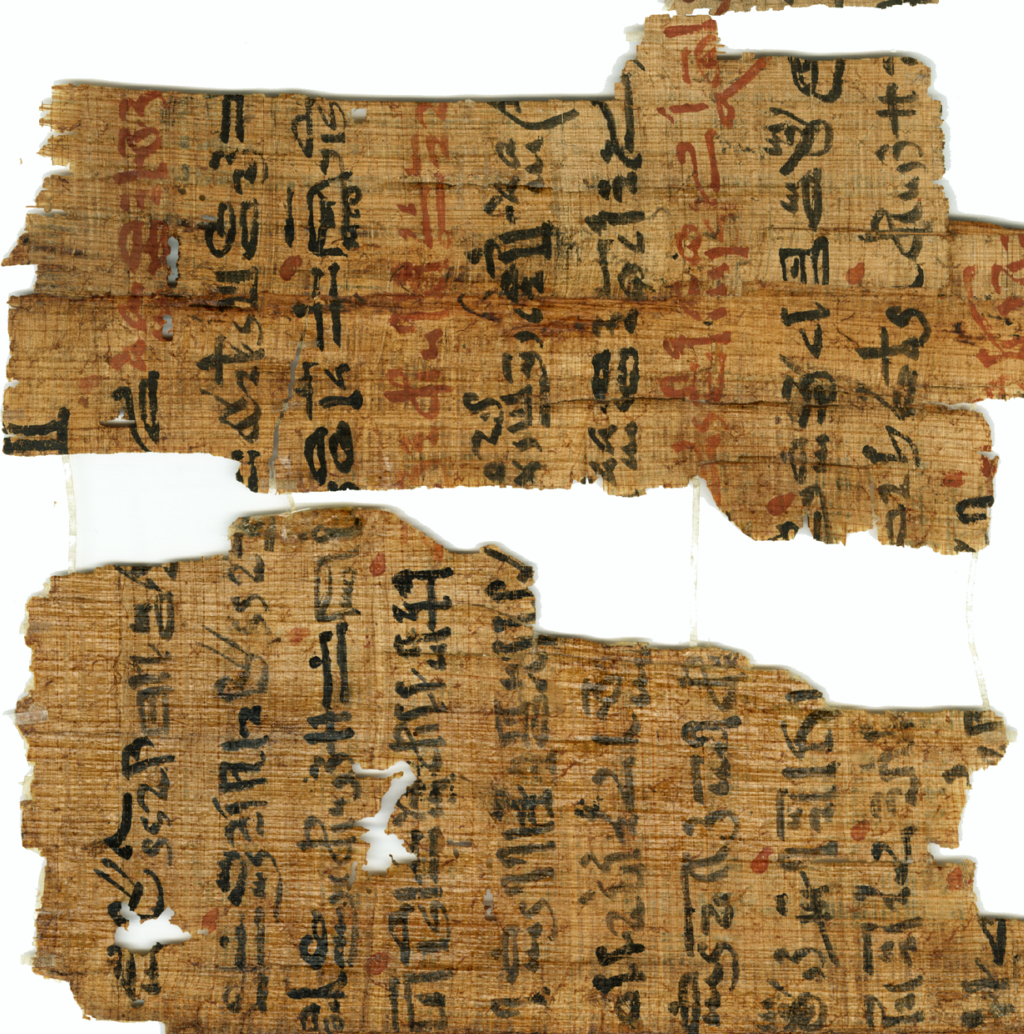


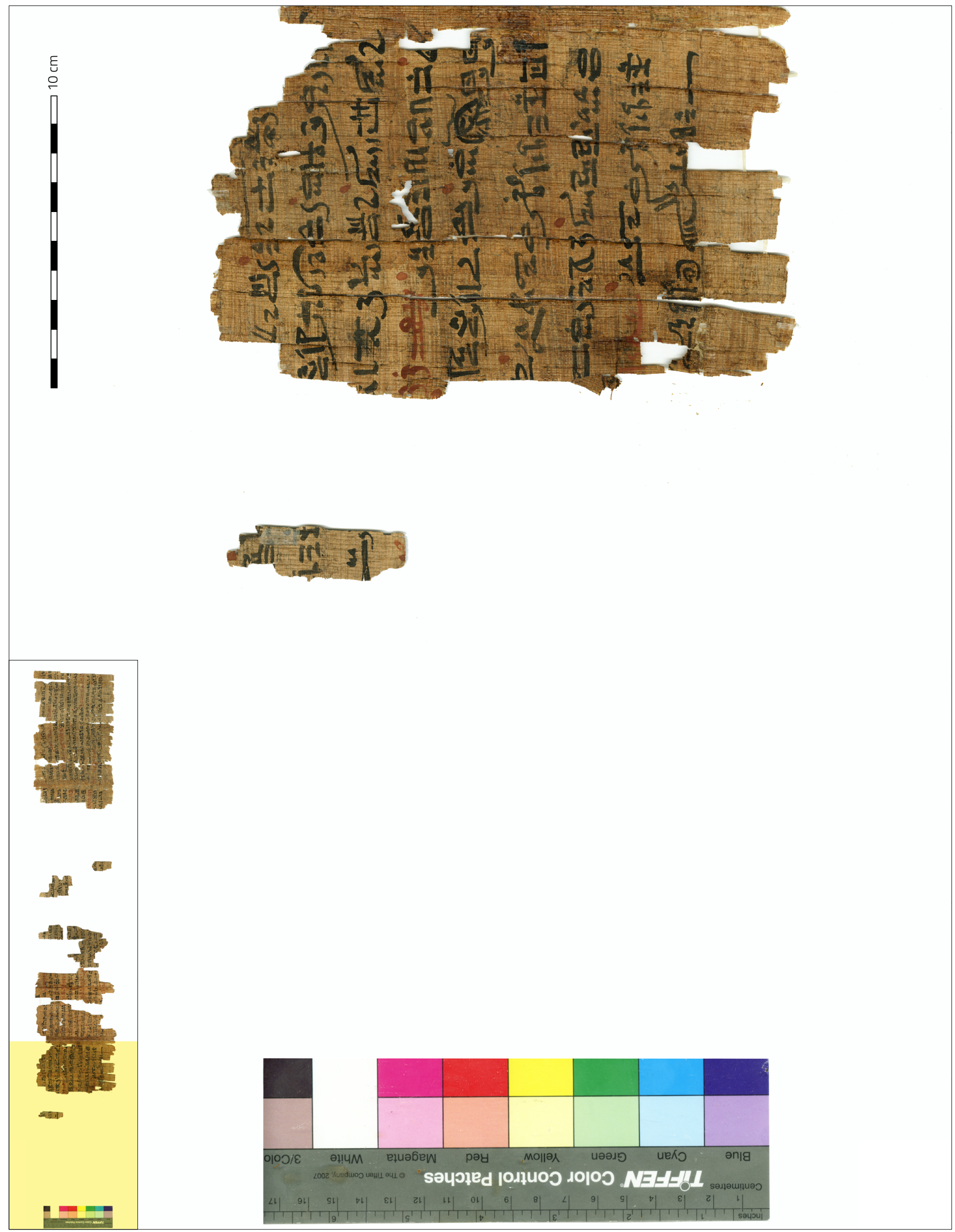

PI. 2 : Pap. CGT 54018 rº. Image numérisée : Museo Egizio. Remontage final de l'auteur. 


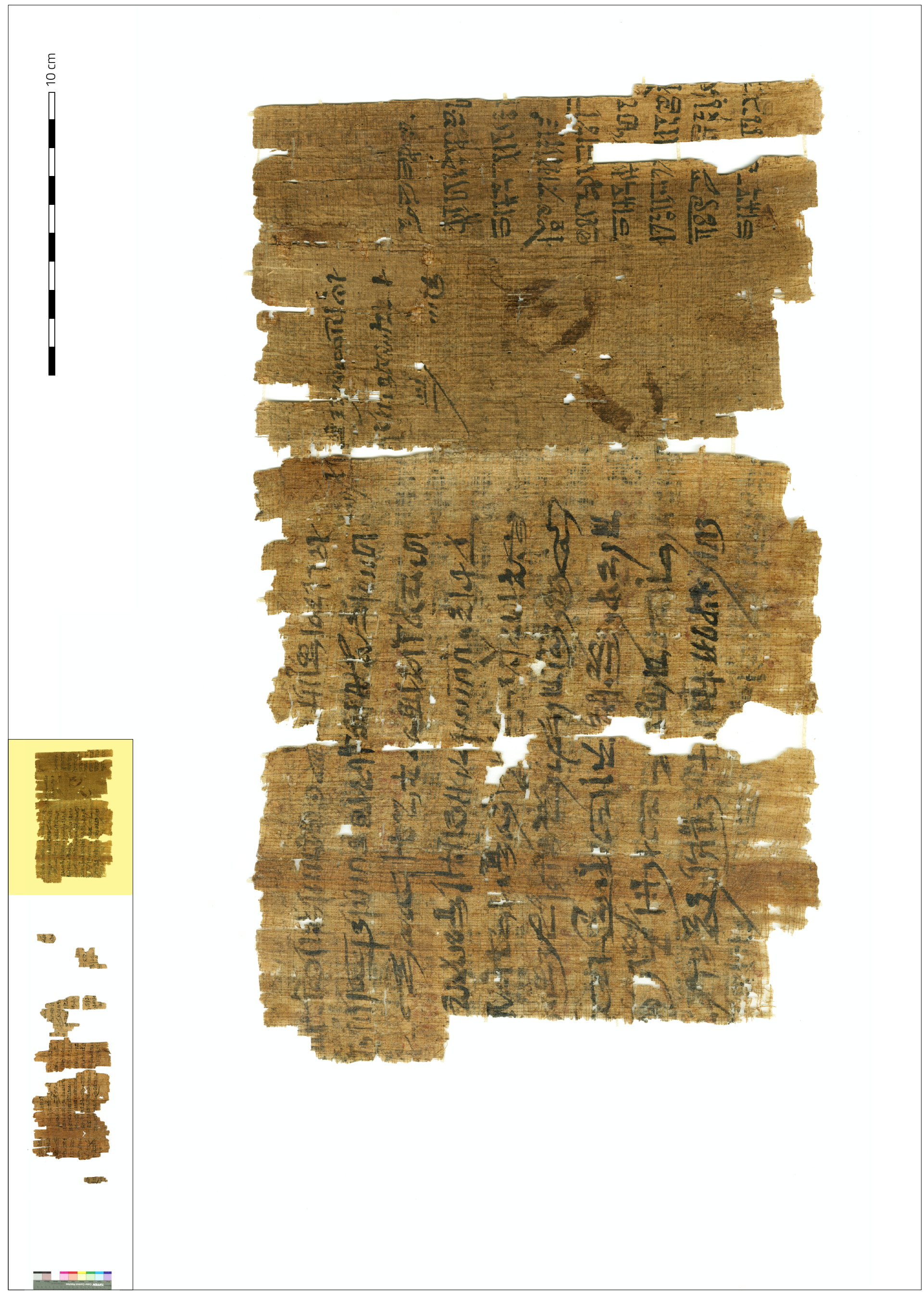




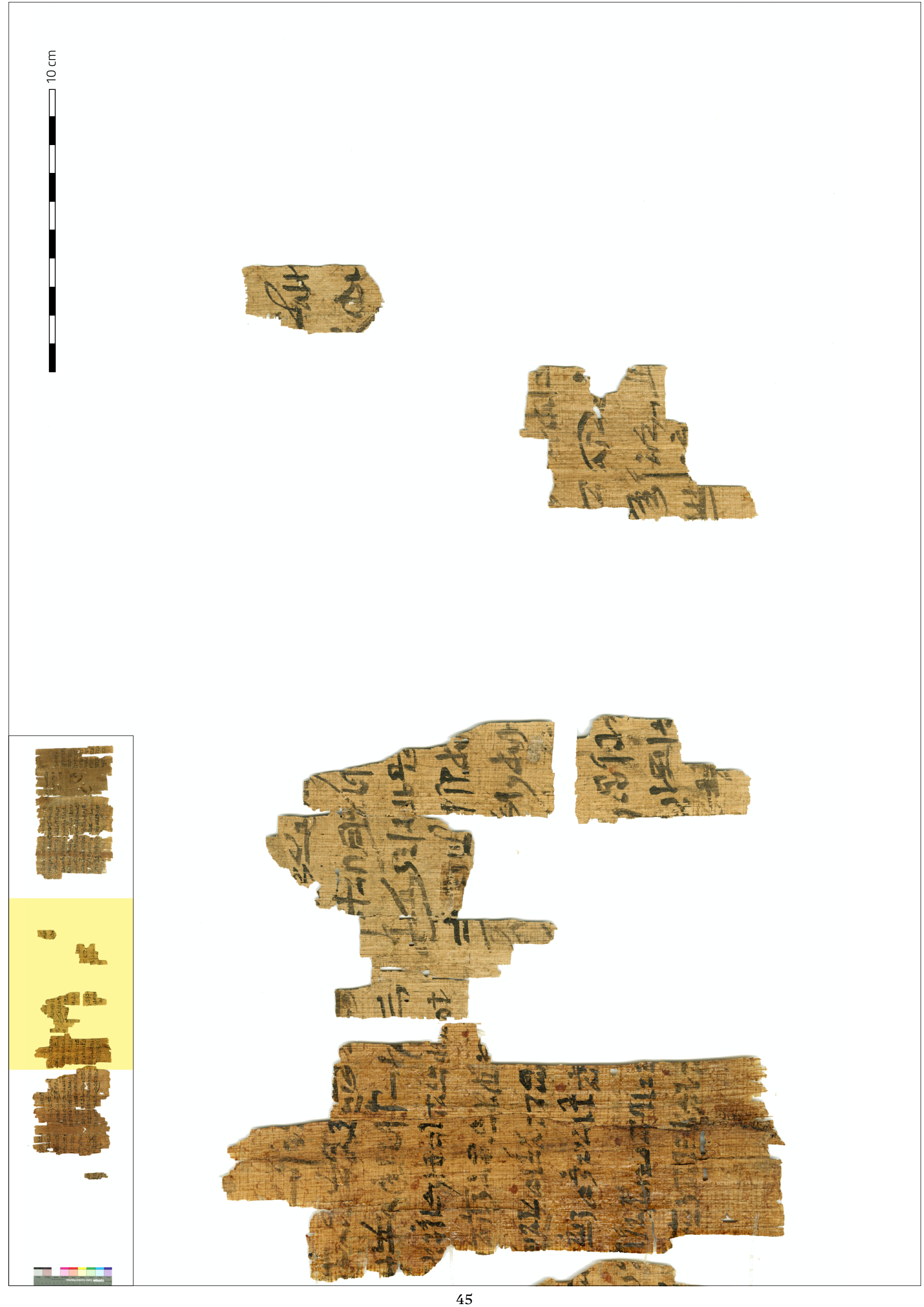






PI. 3 : Pap. CGT 54018 vº. Image numérisée : Museo Egizio. Remontage final de l'auteur. 


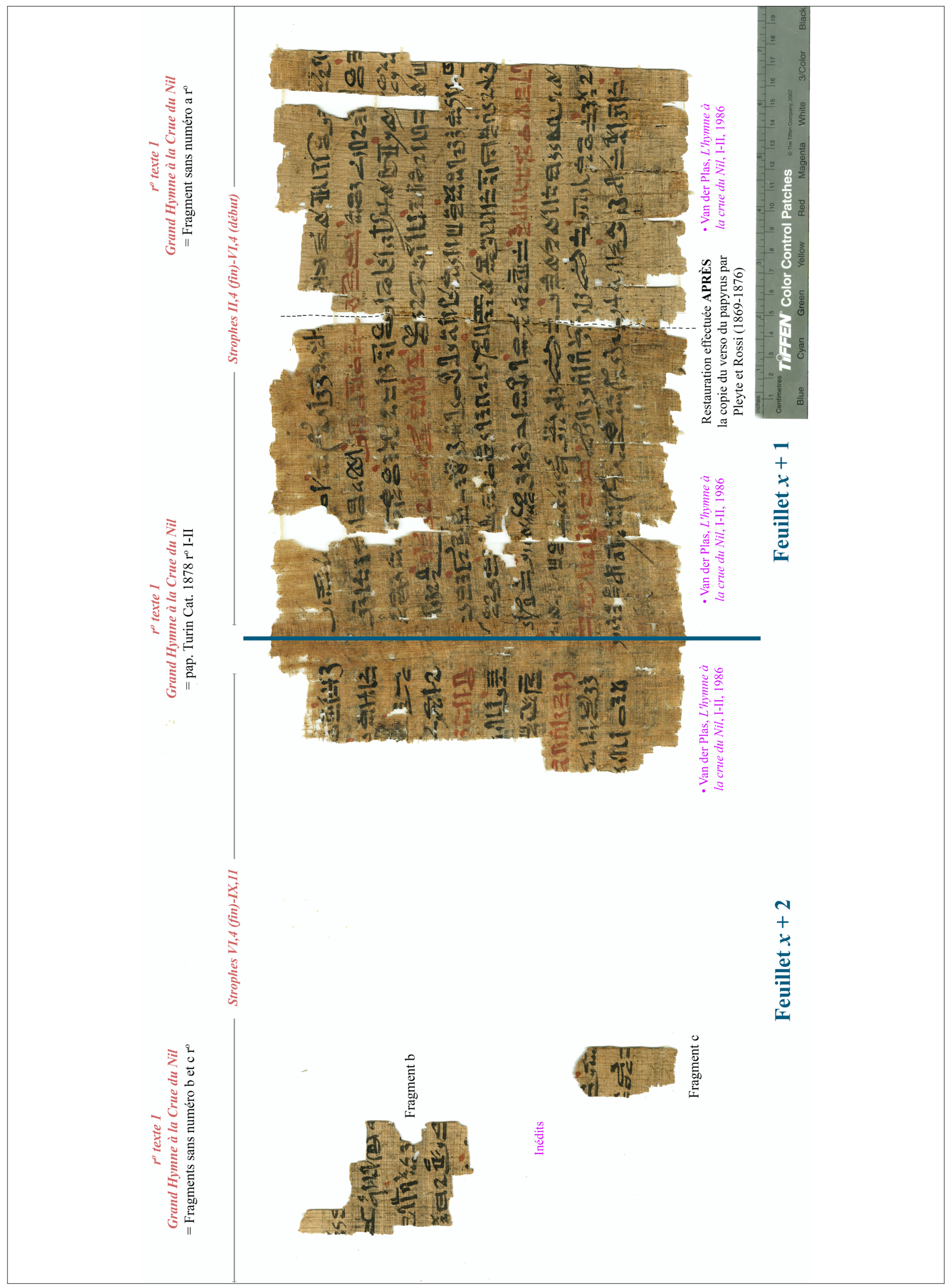




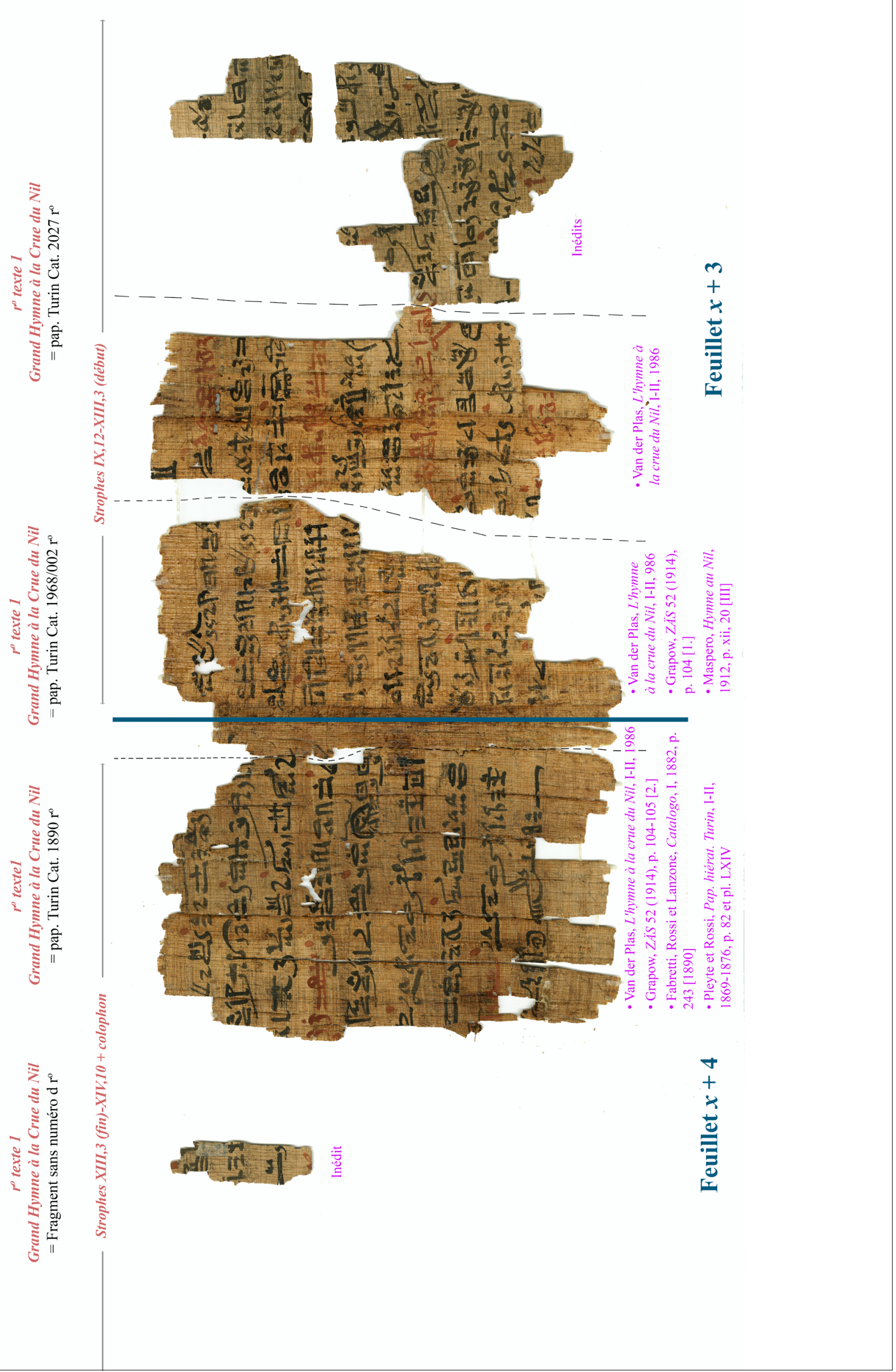

PI. 4 : Pap. CGT 54018 rº, cartographie de la répartition du texte. Image numérisée : Museo Egizio. Remontage final de l'auteur. 


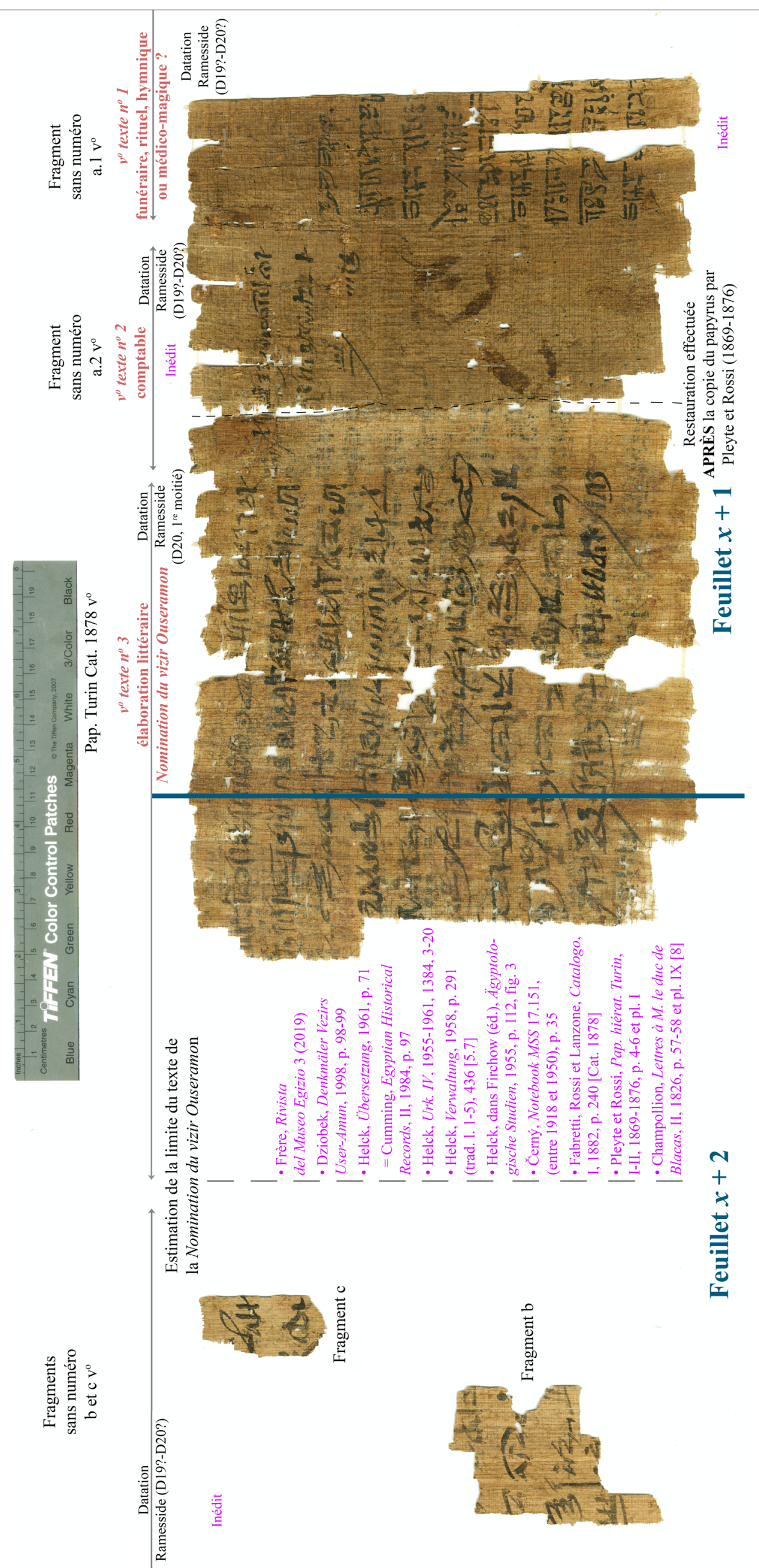




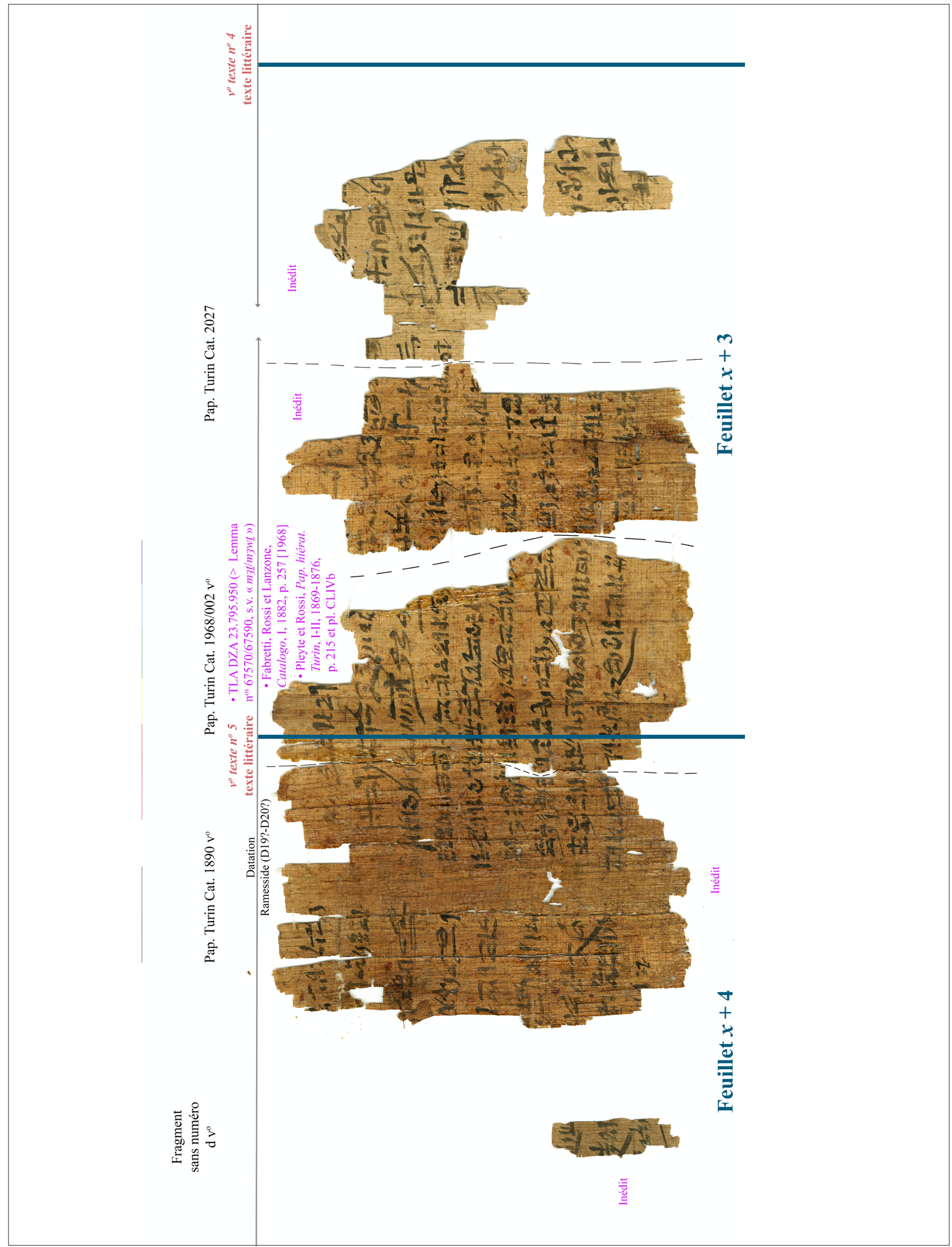

PI. 5 : Pap. CGT 54018 vº cartographie de la répartition des textes. Image numérisée : Museo Egizio. Remontage final de l'auteur. 


\section{Annexe 1}

\section{Paléographie du Papyrus Turin cat. 1878 v$^{0}$, l. 1-9}

Le tableau ci-dessous présente tous les signes hiératiques présents sur le papyrus Turin Cat. $1878 \mathrm{v}^{\mathrm{o}}$, 1. 1-9 afin de répondre à deux objectifs principaux : d'une part confirmer l'hypothèse de datation ramesside, et apporter, le cas échéant, une précision chronologique ; d'autre part mettre en évidence les particularités et les variations graphiques du scribe. Ces deux paramètres permettront éventuellement d'identifier une « main » déjà connue au sein de la communauté de lettrés de Deir al-Medineh.

La localisation de chaque signe est indiquée par le numéro de la ligne dans laquelle il figure. S'il y a lieu, lorsqu'un même signe est présent à plusieurs reprises sur une même ligne, un nombre entre parenthèses indique son ordre d'apparition dans la ligne en question (exemple pour le signe A1: $3(1)=$ premier signe A1 tracé à la ligne 3 ).

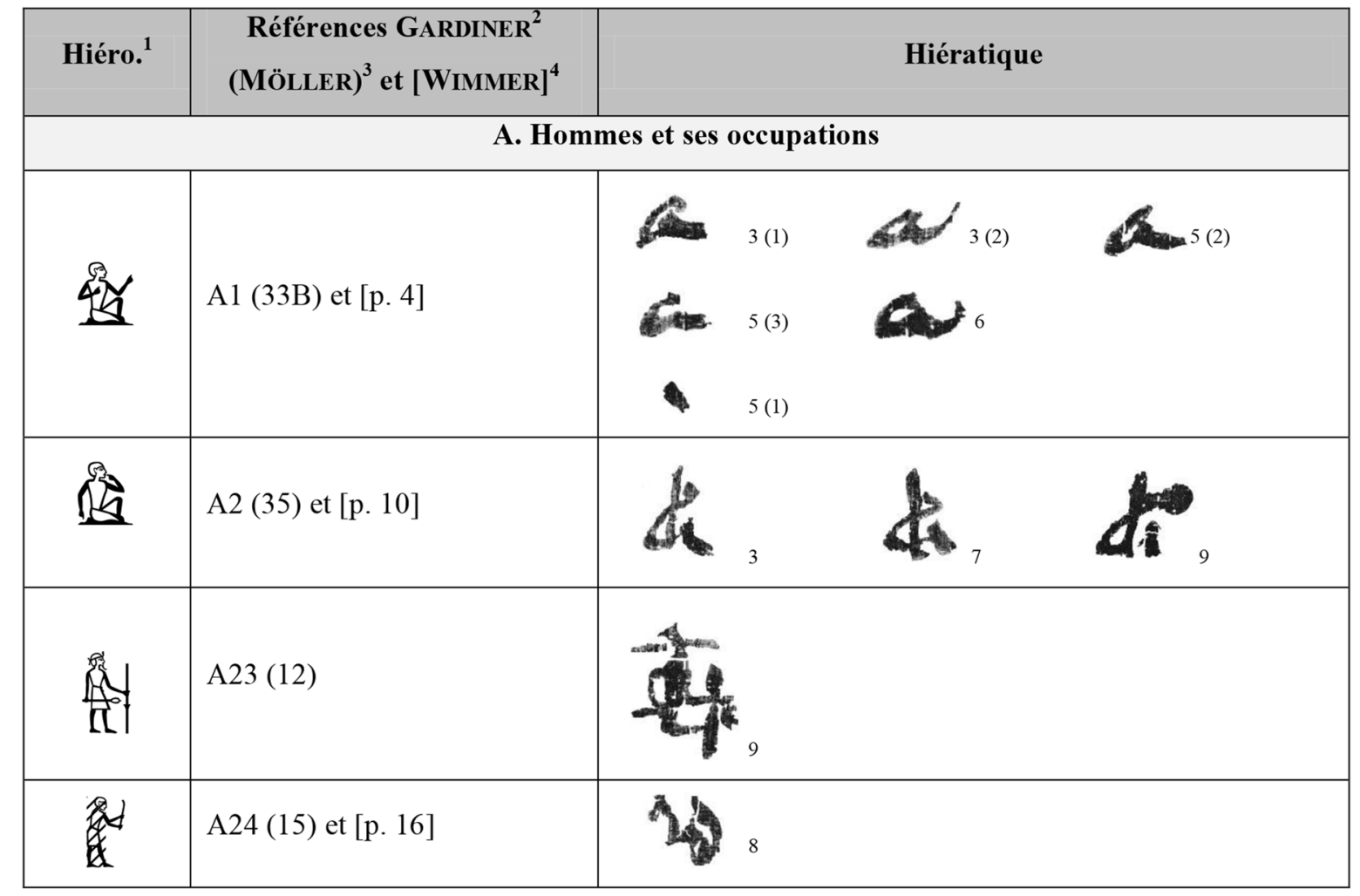

${ }^{1}$ Les hiéroglyphes normalisés sont réalisés avec l'éditeur de textes libre et open source JSesh (http://jsesh.qenherkhopeshef.org/fr) développé par S. Rosmorduc.

${ }^{2}$ Gardiner, Egyptian Grammar, $1957^{3}$, p. 438-548 (Sign-list).

${ }^{3}$ Möller, Hieratische Paläographie, II, 1909-1912.

${ }^{4}$ Wimmer, Hieratische Paläographie, 1995. 


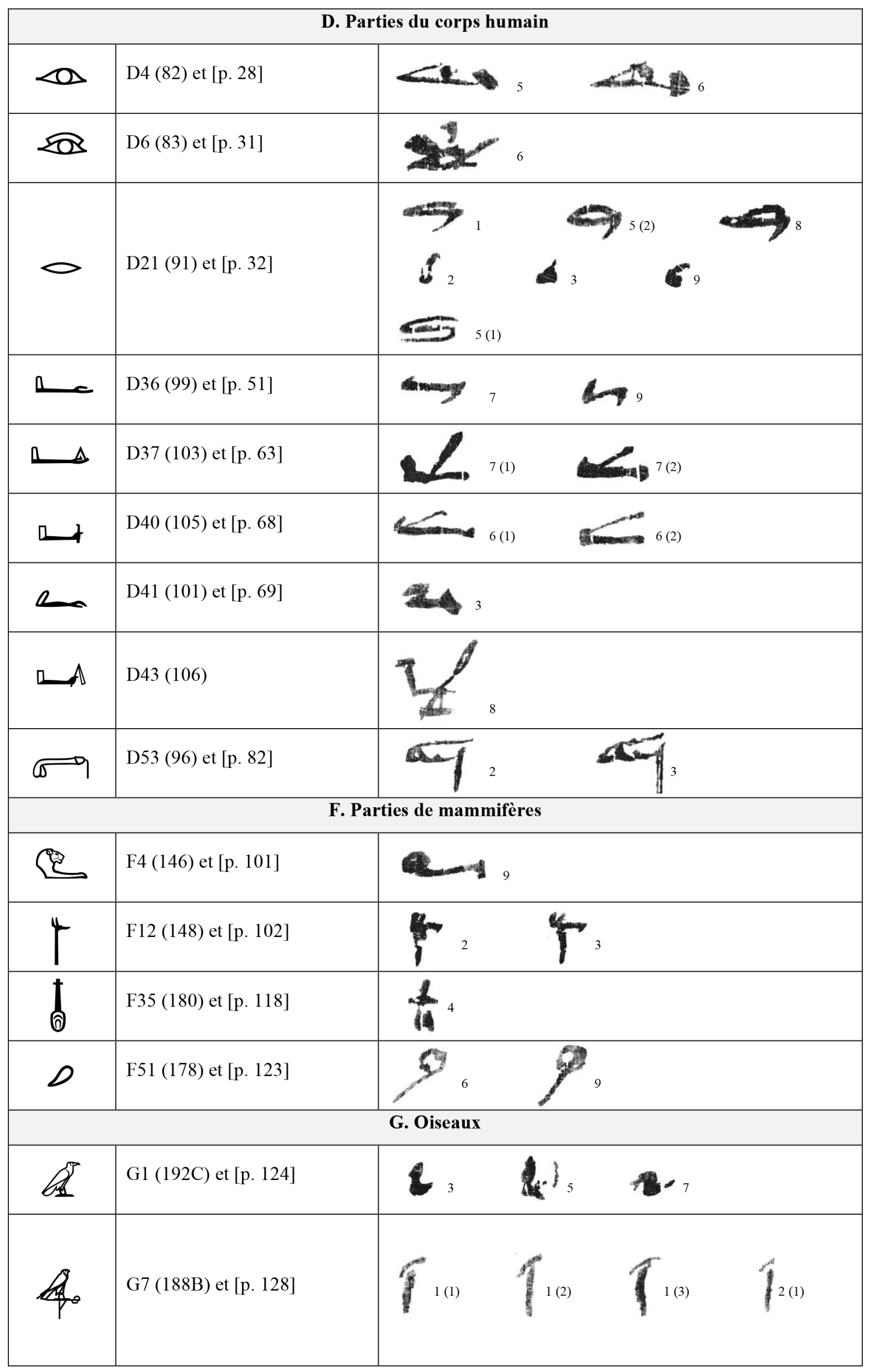

52 


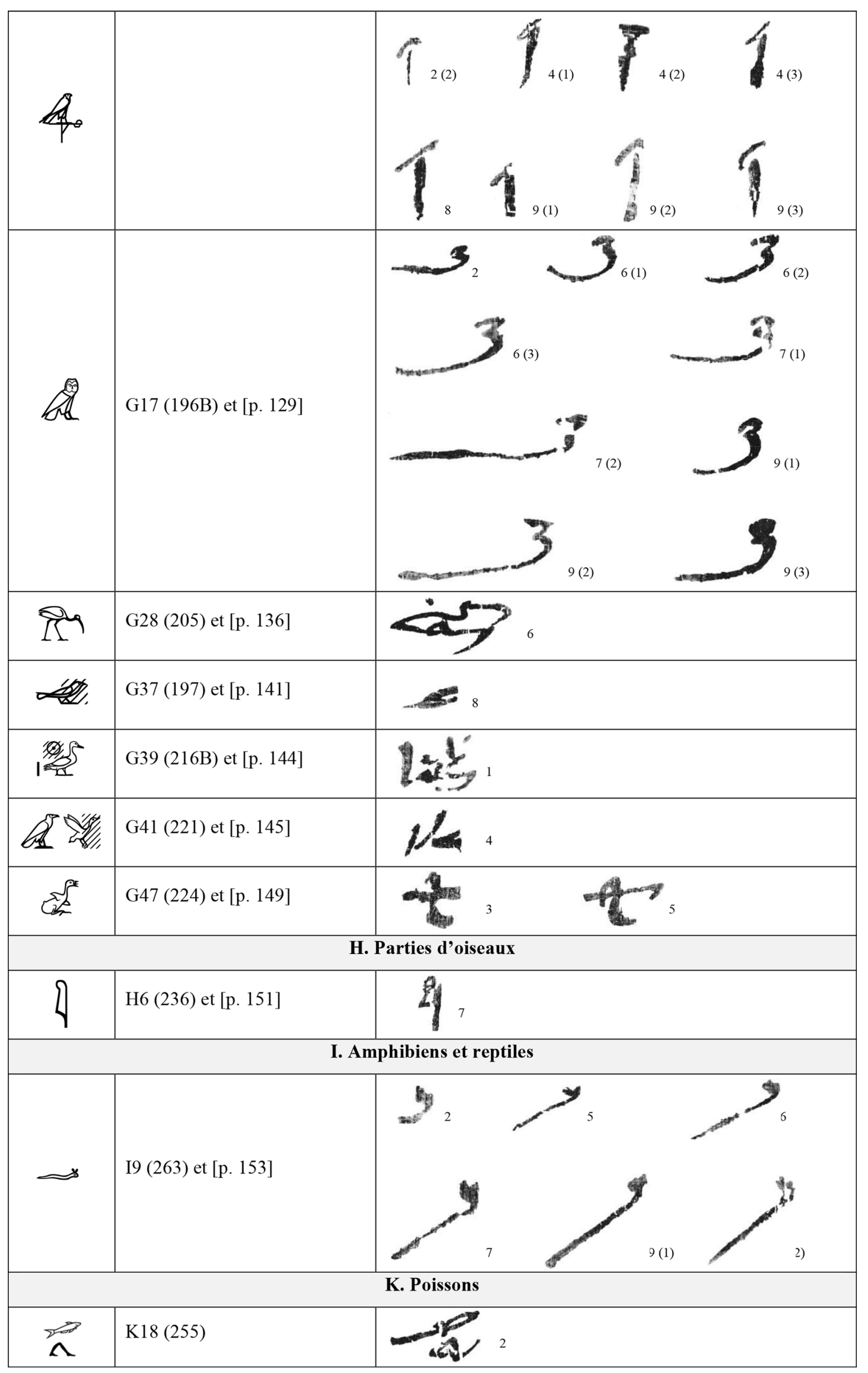

53 


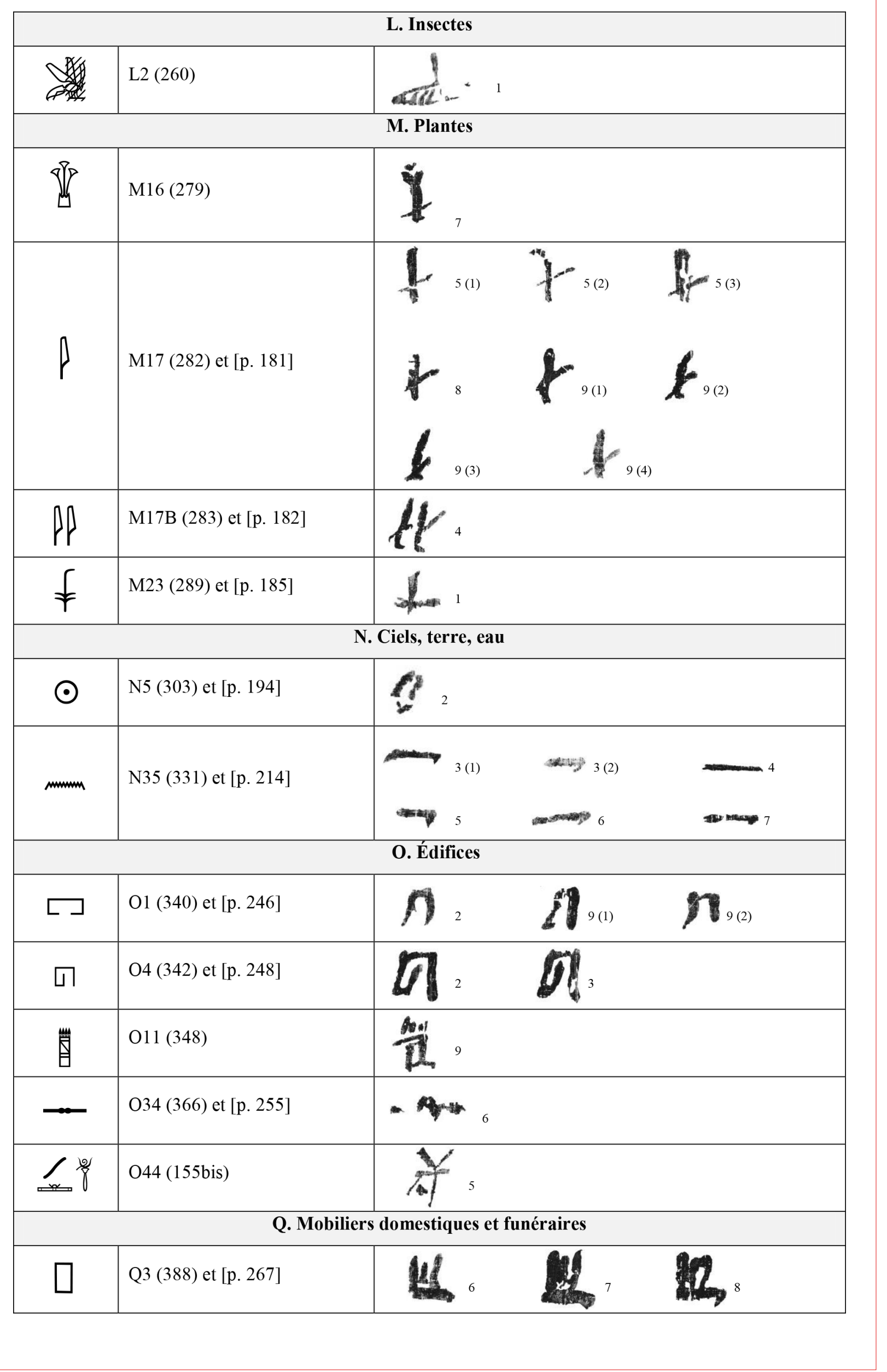




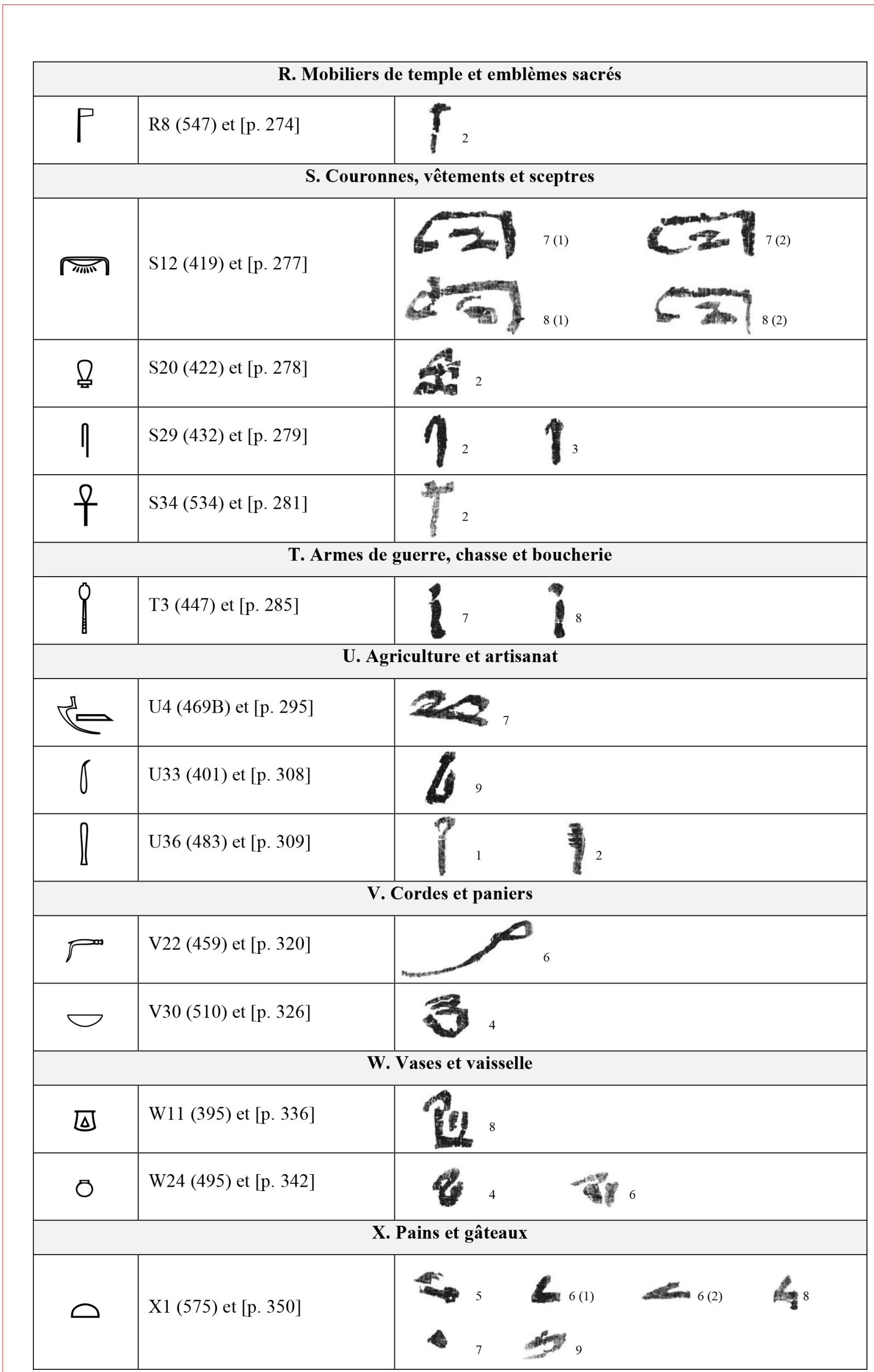

55 


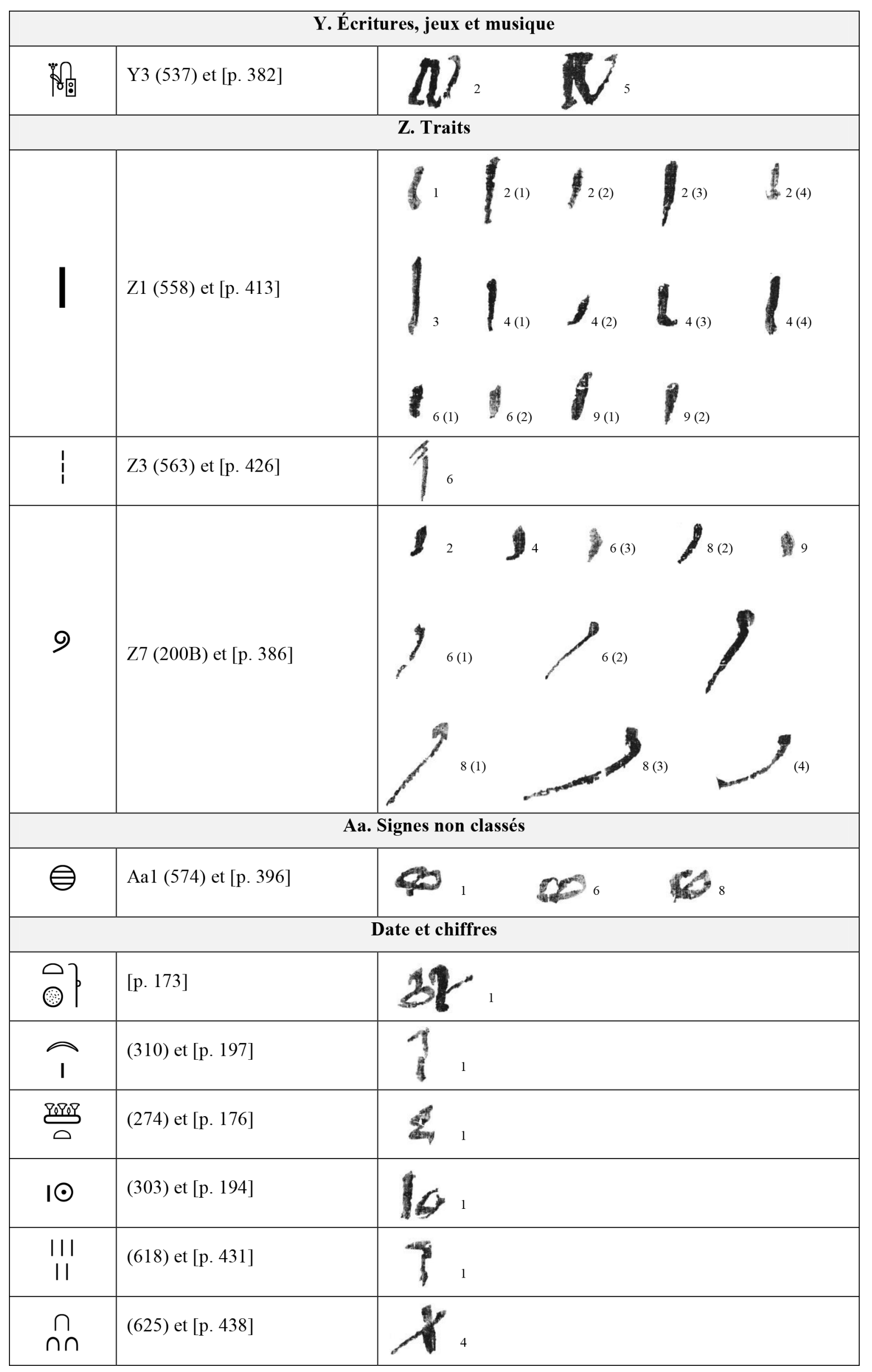

56 


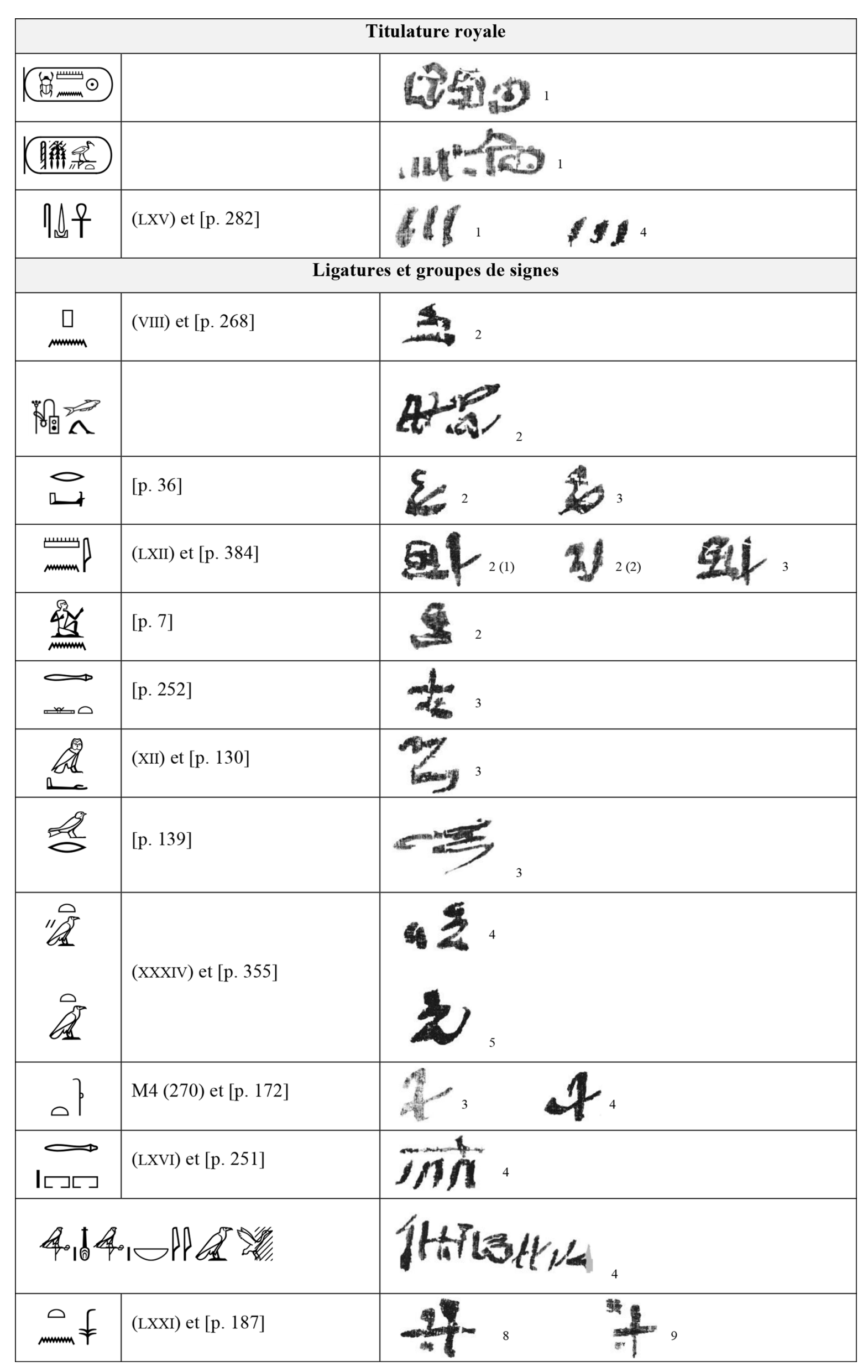




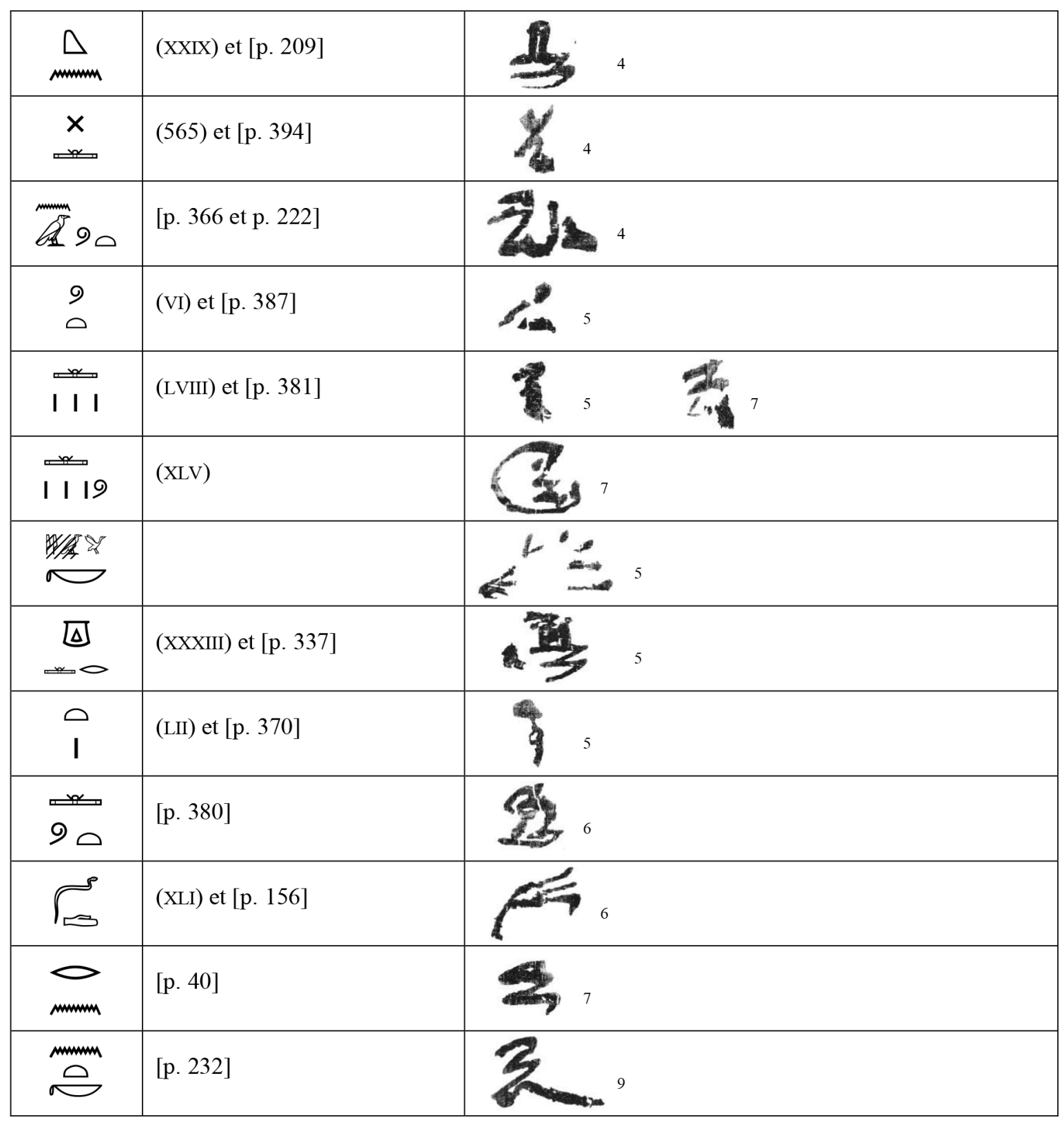

58 